\title{
3D Fluorescence Characterization of Synthetic Organic Dyes_Effect of pH
}

\author{
Leonard J. Soltzberg' ${ }^{1}$, Sarah Flynn ${ }^{1}$, Vera Kirch ${ }^{1}$, Richard Newman ${ }^{2}$ \\ ${ }^{1}$ Department of Chemistry \& Physics, Simmons College, Boston, USA \\ ${ }^{2}$ Scientific Research Lab, Museum of Fine Arts, Boston, USA \\ Email: lsoltzberg@simmons.edu
}

Received August 5, 2013; revised September 5, 2013; accepted September 20, 2013

Copyright (C) 2013 Leonard J. Soltzberg et al. This is an open access article distributed under the Creative Commons Attribution License, which permits unrestricted use, distribution, and reproduction in any medium, provided the original work is properly cited.

\begin{abstract}
The fingerprint character and high sensitivity of 3D UV-vis fluorescence spectra offer special advantages for identification of dyes in a museum or forensic setting. However, the extraction process is likely to affect the $\mathrm{pH}$ of the medium and, in some cases, may alter the dye itself. We report a study of 65 dyes extracted from wool fibers that are part of the Schweppe Collection of Important Synthetic Dyes. The 3D fluorescence spectra of the dye extracts at pH 1 and pH 14 are compared with the same dyes from the Schweppe solution library, run under the same conditions, as well as with the 3D fluorescence spectra of the dyes taken directly from the solution library without $\mathrm{pH}$ control. This analysis leads to guidelines for the use of such spectra in identifying unknown dye samples.
\end{abstract}

Keywords: Fluorescence; Spectrophotometry; Dye Analysis; Textile Analysis

\section{Introduction}

We recently reported "fingerprint" patterns obtained from 3-dimensional fluorescence spectrometry [1] for the 65 synthetic organic dyes in the Schweppe dye collection [2]. To test the practical utility of such patterns for identifying dyes, we studied the 3D fluorescence spectra of extracts of these same dyes from woolen fibers, also from the Schweppe collection.

Because of the trifluoroacetic acid extraction procedure, we employed results in a highly acidic solution; we examined the effect of $\mathrm{pH}$ on the 3D fluorescence spectra of these dyes and found that many of the spectra are $\mathrm{pH}$ dependent. Therefore, we developed a standard protocol in which the fluorescence spectrum of each dye extract is recorded at $\mathrm{pH} 1$ and then again at $\mathrm{pH} 14$. Dye samples from the Schweppe solution library were subjected to the same protocol, and their fluorescence spectra were compared with those from the wool extracts. This comparison led to recommendations on the interpretation of such spectra for problems involving dye identification.

\section{Experimental}

\subsection{Instrumentation}

We used a Hitachi F 4500 fluorescence spectrophotometer with a Hitachi 650 - 0116 microcell for running fluo- rescence spectra; this cell requires $200 \mu \mathrm{L}$ of solution. The excitation wavelength EX was scanned from 250 $380 \mathrm{~nm}$ and the emission wavelength EM was scanned from $395-700 \mathrm{~nm}$. These two ranges intentionally do not overlap in order to avoid exposing the photomultiplier to $1^{\text {st }}$ order scattering from the Xe light source. The scanning rate was $1200 \mathrm{~nm} / \mathrm{min}$, and the response time was set at $0.1 \mathrm{sec}$. With these conditions, a spectrum could be obtained in less than 8 minutes with no apparent loss of detail, compared with slower scan speeds.

Approximate $\mathrm{pH}$ measurements with narrow-range $\mathrm{pH}$ paper were spot-checked and confirmed using a Fisher Accumet Model AB-15 pH meter with an Accumet glass combination electrode (Fisher 13-620-285).

\subsection{Materials}

The original solution Schweppe Library of Synthetic Organic Dyes [2] is held at the J. P. Getty Museum in Los Angeles, CA. The samples from this Library in the possession of the Boston Museum of Fine Arts (MFA) are solutions in methanol. The collection includes strands of wool yarn dyed with these same dyes.

For extracting the dyes, we diluted Aldrich spectrophotometric grade trifluoroacetic acid (TFA) (Aldrich 302031) with Sigma-Aldrich Chromasolv-Plus HPLC water (Sigma-Aldrich 34877) to $2 \mathrm{M}$ and also used 
Sigma-Aldrich Chromasolv HPLC MeOH (Sigma-Aldrich 34860). For adjusting the $\mathrm{pH}$, we made a $6 \mathrm{M}$ solution of $\mathrm{NaOH}$ using Fisher NF/FCC sodium hydroxide (Fisher S 320) and HPLC water. 100\% pure medical grade USP modified lanolin (CVS Lanolin Cream, SKU 148868) was used for the lanolin spectrum, as described below.

Since the original Schweppe collection is over 50 years old, some of the library solutions show obvious decomposition, indicated by an absence of color: Acid Black 1 (CI 20470), Basic Green 4 (CI 42000), Acid Blue 93 (CI 42780), Acid Black 2 (CI 50420), Murexide (CI 56085), Mordant Red 3 (CI 58005), and Acid Blue 74 (CI 73015). For these dyes, the fluorescence spectra reported were obtained using fresh dye samples.

The issue of dye concentration has been discussed in [1].

\subsection{Extraction of the Dyes}

The extraction of both synthetic and natural dyes from textile samples for analysis has been a subject of prior investigation [see for example 3 and 4]. Souto [3] compared a variety of extraction methods for sampling synthetic organic dyes from textiles. That work suggests that dilute TFA is the most effective extracting agent for most of the dyes studied. $\mathrm{HCl} /$ methanol is also commonly used $[4,5]$. While Souto removed the TFA after extraction by heating under vacuum, we found that the resulting material was still acidic. Since dye spectra may be $\mathrm{pH}$ dependent and, indeed, a number of the Schweppe dyes are actually $\mathrm{pH}$ indicators, we opted to adjust the $\mathrm{pH}$ to standard levels rather than attempting to remove the TFA, thus providing well-defined protonated and deprotonated dye species for fluorometric analysis.

To extract a sample, we clipped a fragment of wool (about $2 \mathrm{mg}$ ) from a Schweppe library wool sample and placed it at the bottom of a $0.5 \mathrm{~mL}$ polypropylene microcentrifuge tube. We added $20 \mu \mathrm{L} 2 \mathrm{M}$ TFA, agitated with a glass rod, and let stand at room temperature for $2 \mathrm{~min}$. We then added $30 \mu \mathrm{L} \mathrm{MeOH}$ and let stand for another 4 minutes at room temperature. The sensitivity of fluorescence spectrometry is such that sufficient dye was extracted in most cases even under these gentle conditions. Finally, the extract was diluted with $250 \mu \mathrm{L} \mathrm{H}_{2} \mathrm{O}$. The dyes insufficiently extracted under these conditions were Acid Violet 7 (CI 18055), Acid Black 1 (CI 20470), Basic Yellow 2 (CI 41000), Acid Green 6 (CI 42075), and Mauveine (CI 50245).

\section{4. pH Adjustment}

The dye solution as extracted from the wool fibers with TFA is highly acidic, even after dilution, with a $\mathrm{pH}$ of 1 indicated by narrow range $\mathrm{pH}$ paper and confirmed using a glass electrode $\mathrm{pH}$ meter. After the 3D fluorescence spectrum was run at $\mathrm{pH} 1,10 \mu \mathrm{L}$ of $6 \mathrm{M} \mathrm{NaOH}$ solution was added to the microcuvette and thoroughly mixed. If necessary, additional $\mathrm{NaOH}$ solution was added to bring the $\mathrm{pH}$ to 14 . The $\mathrm{pH} 14$ spectrum was then run.

\section{Results and Discussion}

The Supplementary Information-Table 1

[http://simmons.academia.edu/LenSoltzberg] contains, for each of the 46 Schweppe dyes giving a usable match between the wool extract and library spectra, the 3D fluorescence spectra of the wool extracts and library solutions at $\mathrm{pH} 1$ and $\mathrm{pH}$ 14, along with the unbuffered library spectrum and corresponding molecular structure. We refer to those spectra using the Schweppe number; e.g., "S10". Spectra illustrating particular findings are given in the figures below.

\subsection{Spectral Artifacts}

Certain distinct artifacts characteristic of fluorescence spectra need to be taken into account when examining the spectra reported here. These artifacts include Rayleigh scattering and Raman scattering from the solvent. These artifacts have been discussed in detail in [1] but are shown again here in Figure $\mathbf{1}$ to facilitate reading the dye spectra shown in the present work. 3D fluorescence spectra of $\mathrm{pH} 1$ and $\mathrm{pH} 14$ blank solutions showed no features other than these instrumental artifacts.

Care needs to be taken to avoid confusion due to a peak centered near $\lambda_{\mathrm{EX}}=300 \mathrm{~nm} \lambda_{\mathrm{EM}}=400 \mathrm{~nm}$, often seen in the $\mathrm{pH} 14$ wool extract spectra (Figure 2(a)) and apparently due to lanolin (Figure 2(b)). A weak fluorescence spectrum has been previously reported for lanolin [7]. For weakly fluorescent or poorly extracted dyes, the lanolin peak may overlap with or obscure dye features. Examination of the $\mathrm{pH} 14$ dye library spectrum shows whether the dye itself has a peak at that location.

\subsection{Effect of $\mathbf{p H}$ on Dye Spectra}

While a detailed correlation of fluorescence spectra with molecular structure is a complex matter [8], it is no surprise that these spectra can be strongly influenced by $\mathrm{pH}$ [9]. For example, while the 3D fluorescence spectra of S 51 at the $\mathrm{pH} 1 / \mathrm{pH} 14$ extremes are virtually identical (Figure 3), the spectrum of S16 changes markedly with $\mathrm{pH}$ (Figure 4). Protonation/deprotonation of $-\mathrm{OH},-\mathrm{COOH}$, $-\mathrm{SO}_{3} \mathrm{H}$, or $-\mathrm{NH}_{2}$ groups commonly found in synthetic dyes, plus the consequent change in conjugation, may be expected to alter both the excitation and the emission profiles, leading to changes in the $3 \mathrm{D}$ fluorescence pattern.

Of the 65 dyes in the Schweppe collection, the 3D 
Table 1. Dye can be identified from 3D fluorescence spectra.

\begin{tabular}{|c|c|c|}
\hline & Acidic & Basic \\
\hline $\mathbf{S} \mathbf{x}$ & \multicolumn{2}{|c|}{ [CI (Colour Index) Number CI Name] } \\
\hline wool & dye extracted from wool fibers - pH 1 & dye extracted from wool fibers - pH 14 \\
\hline vial & dye from solution library-pH 1 & dye from solution library-pH 14 \\
\hline Sdb & dye from solution library-no $\mathrm{pH}$ adjustment & dye structure \\
\hline S 1 & \multicolumn{2}{|c|}{ [CI 10305 picric acid] } \\
\hline
\end{tabular}
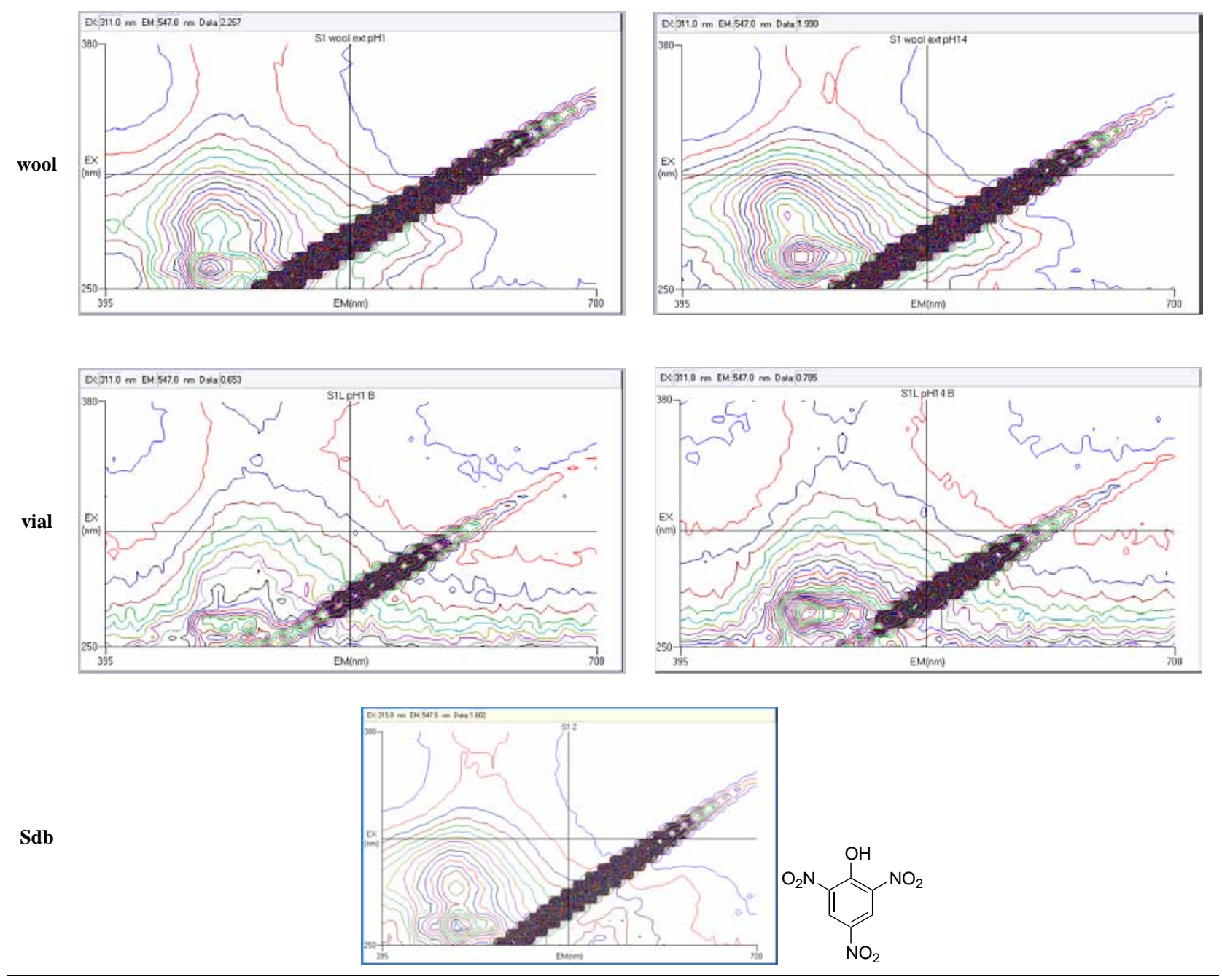

[CI 10316 Acid Yellow 1]
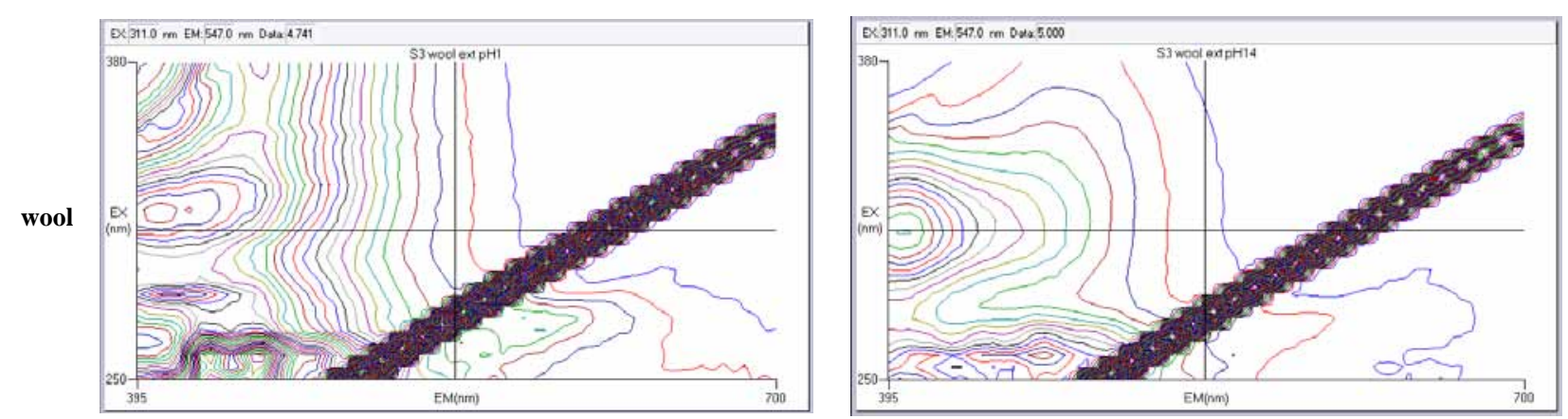


\section{Continued}

vial
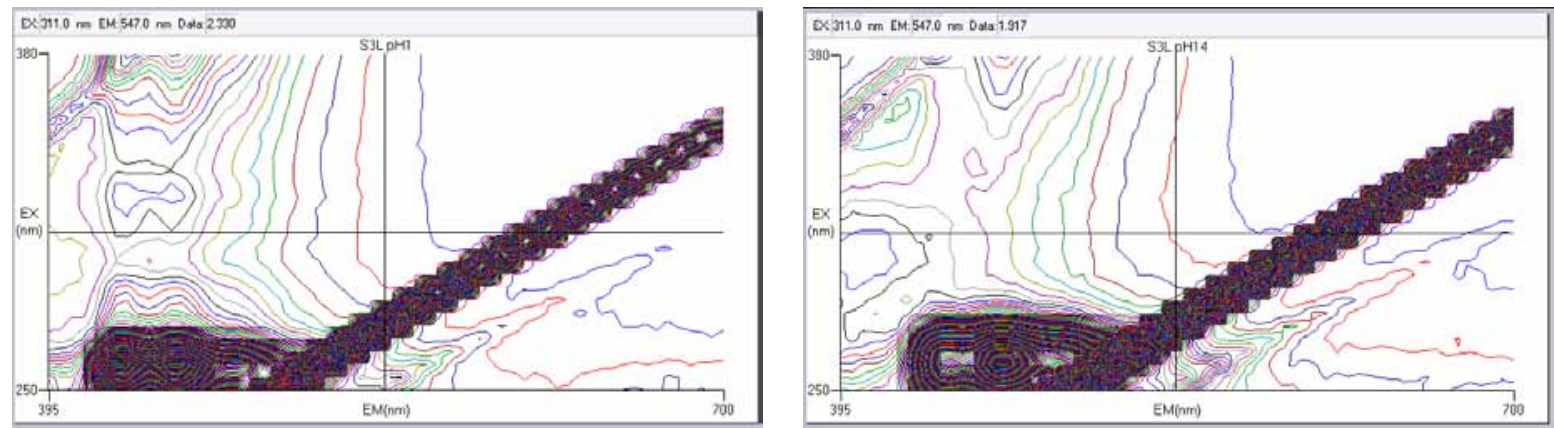

Sdb

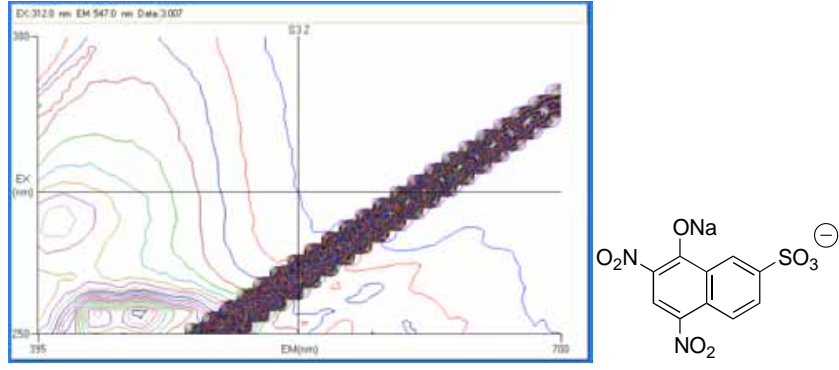

S 4

[CI 11270 Basic Orange 2]
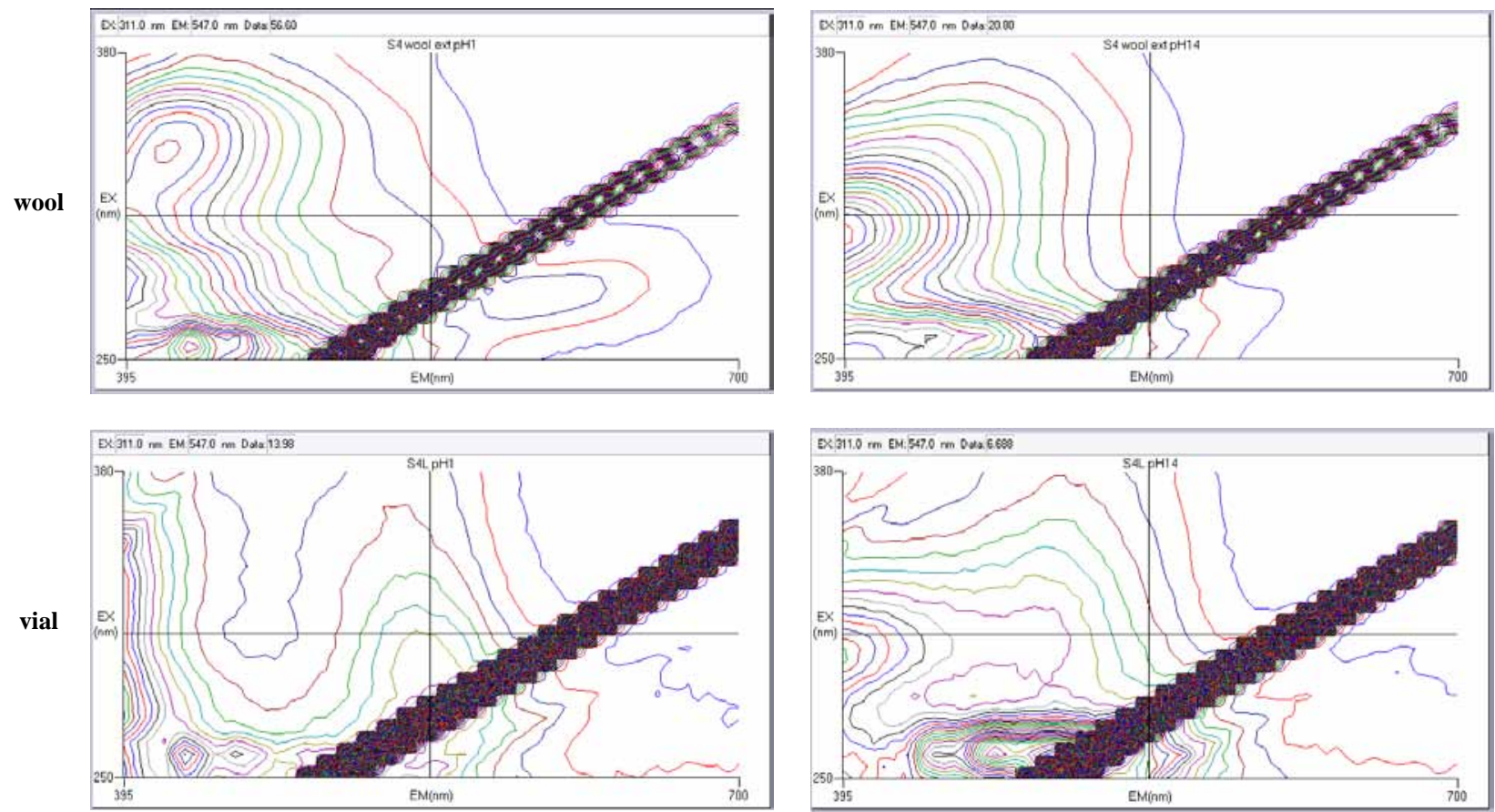

Sdb

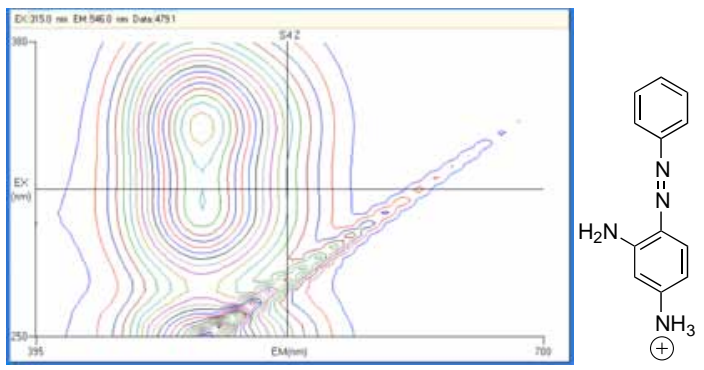




\section{Continued}
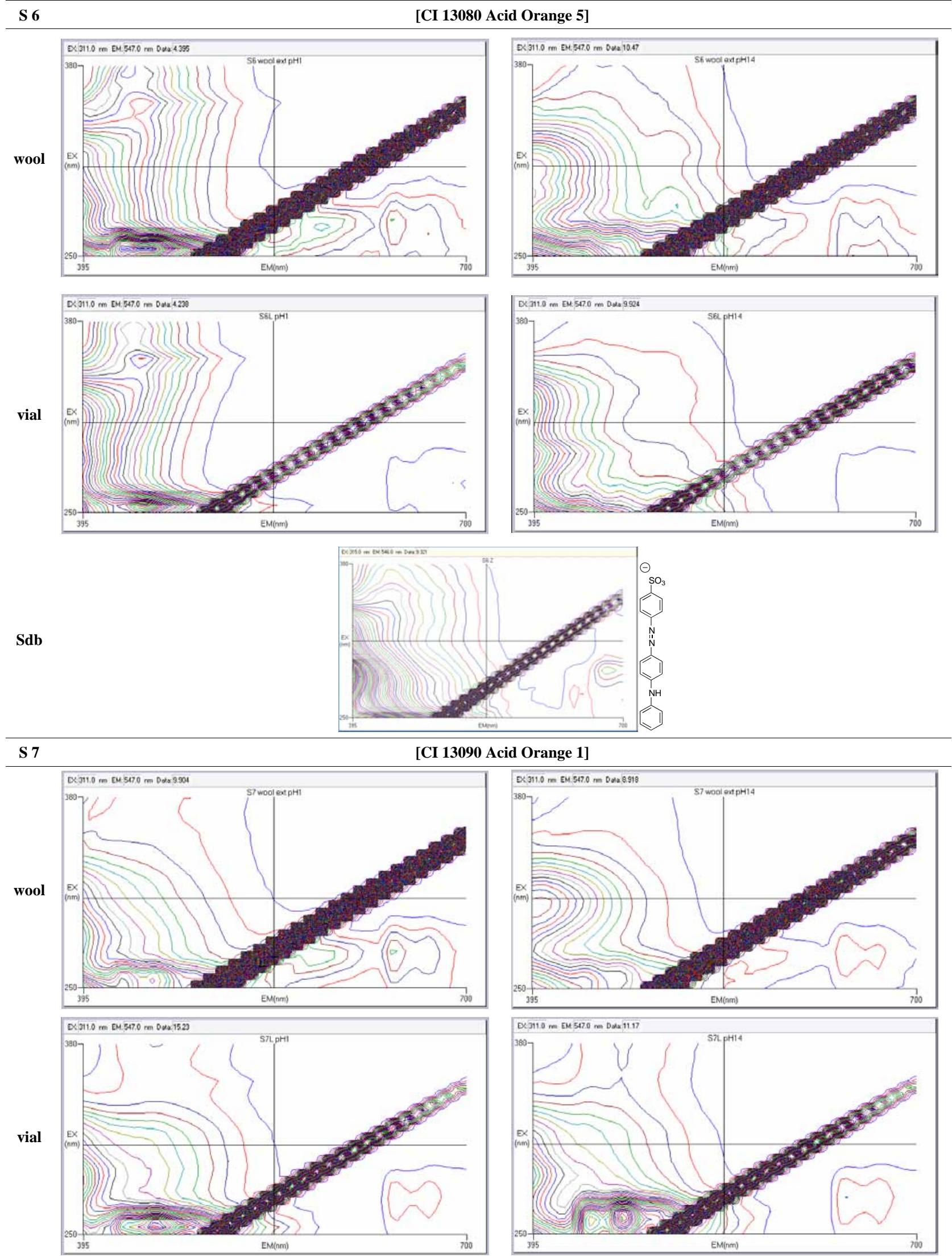

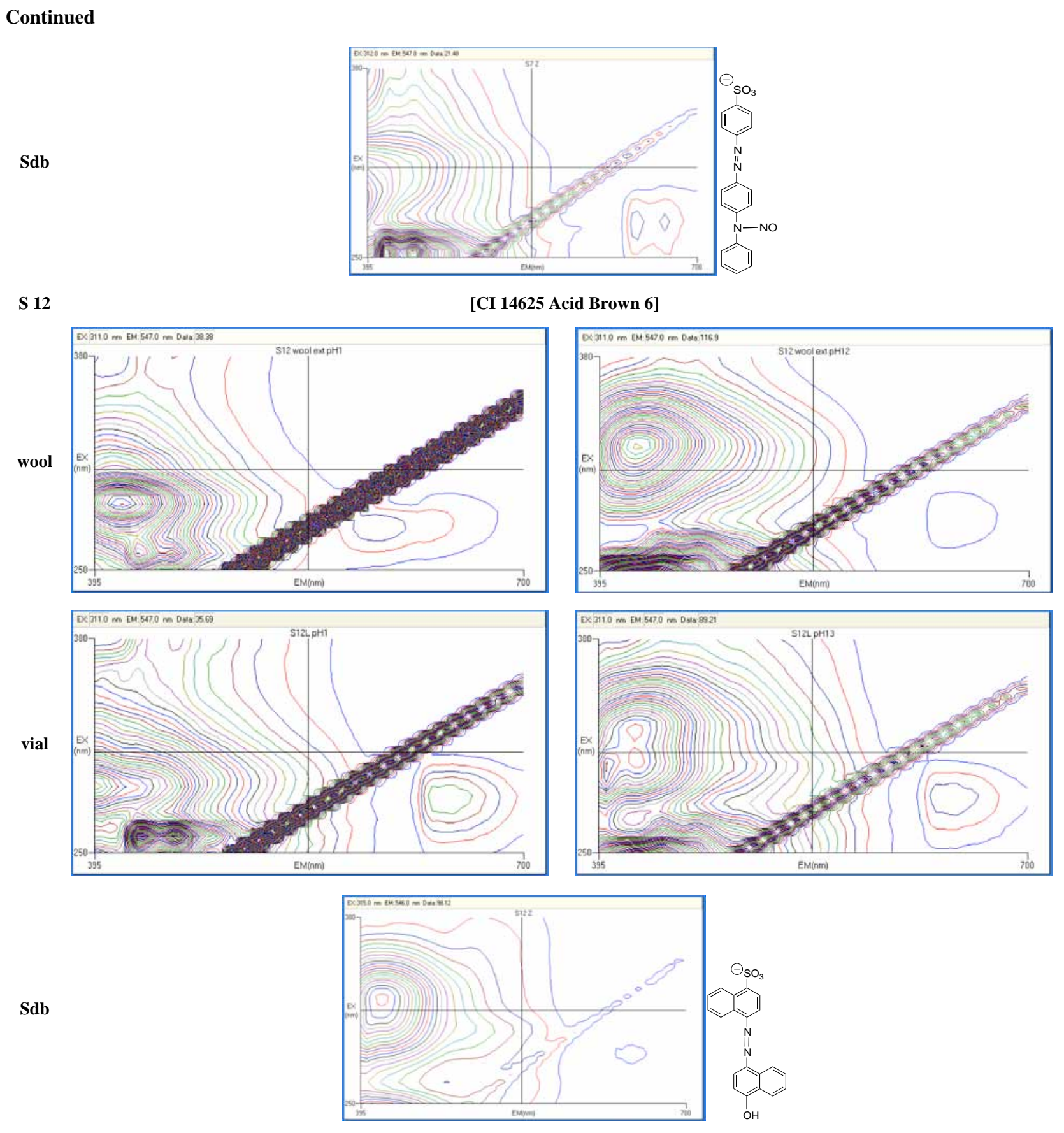

S 14

[CI 15620 Acid Red 88]
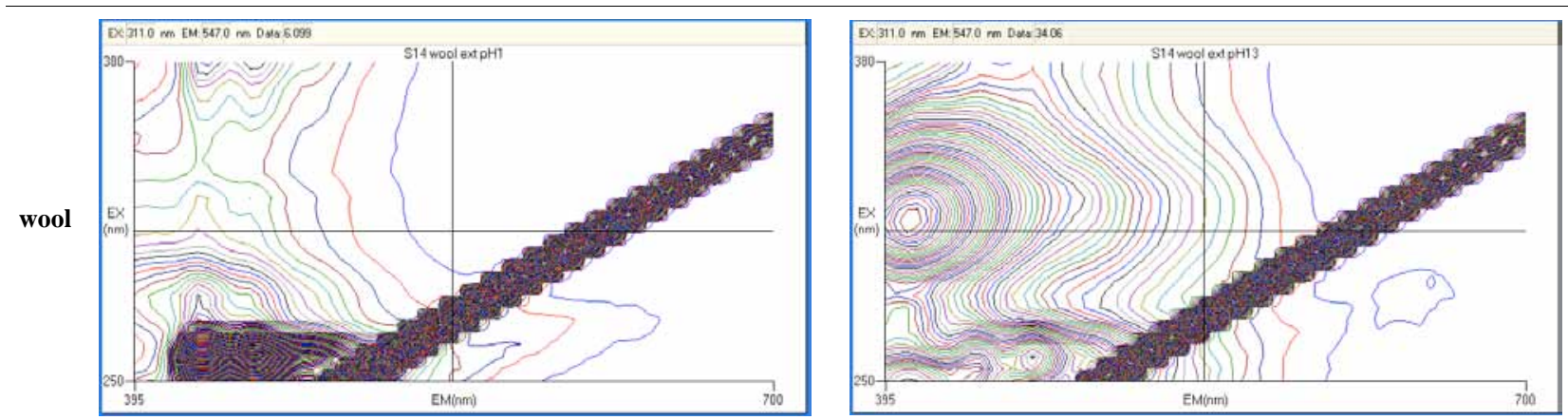


\section{Continued}
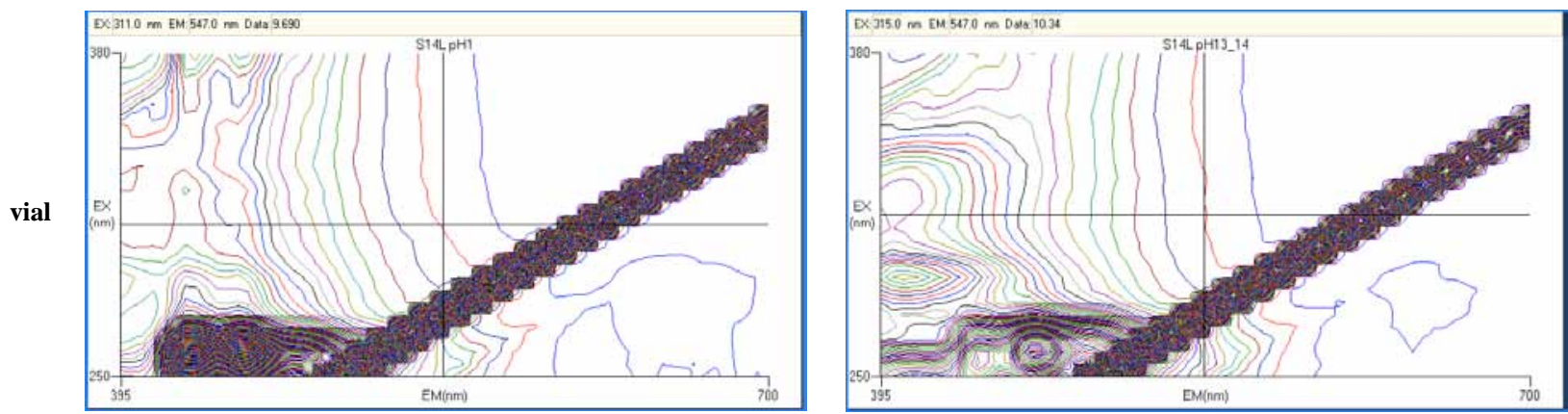

Sdb

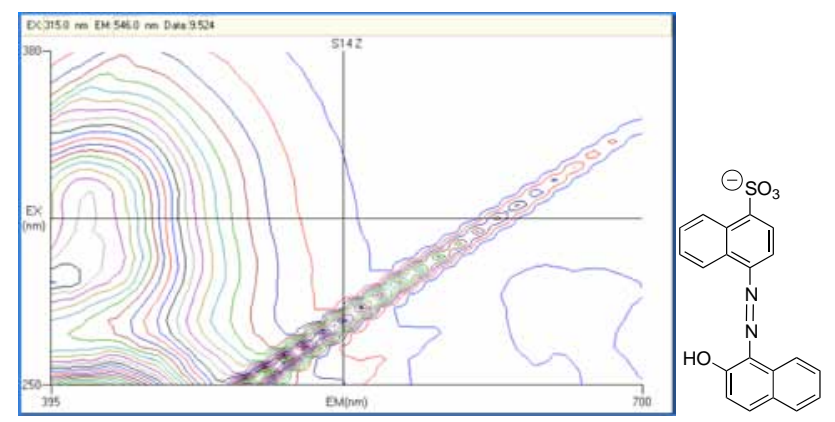

S 15

[CI 15635 Acid Red 9]
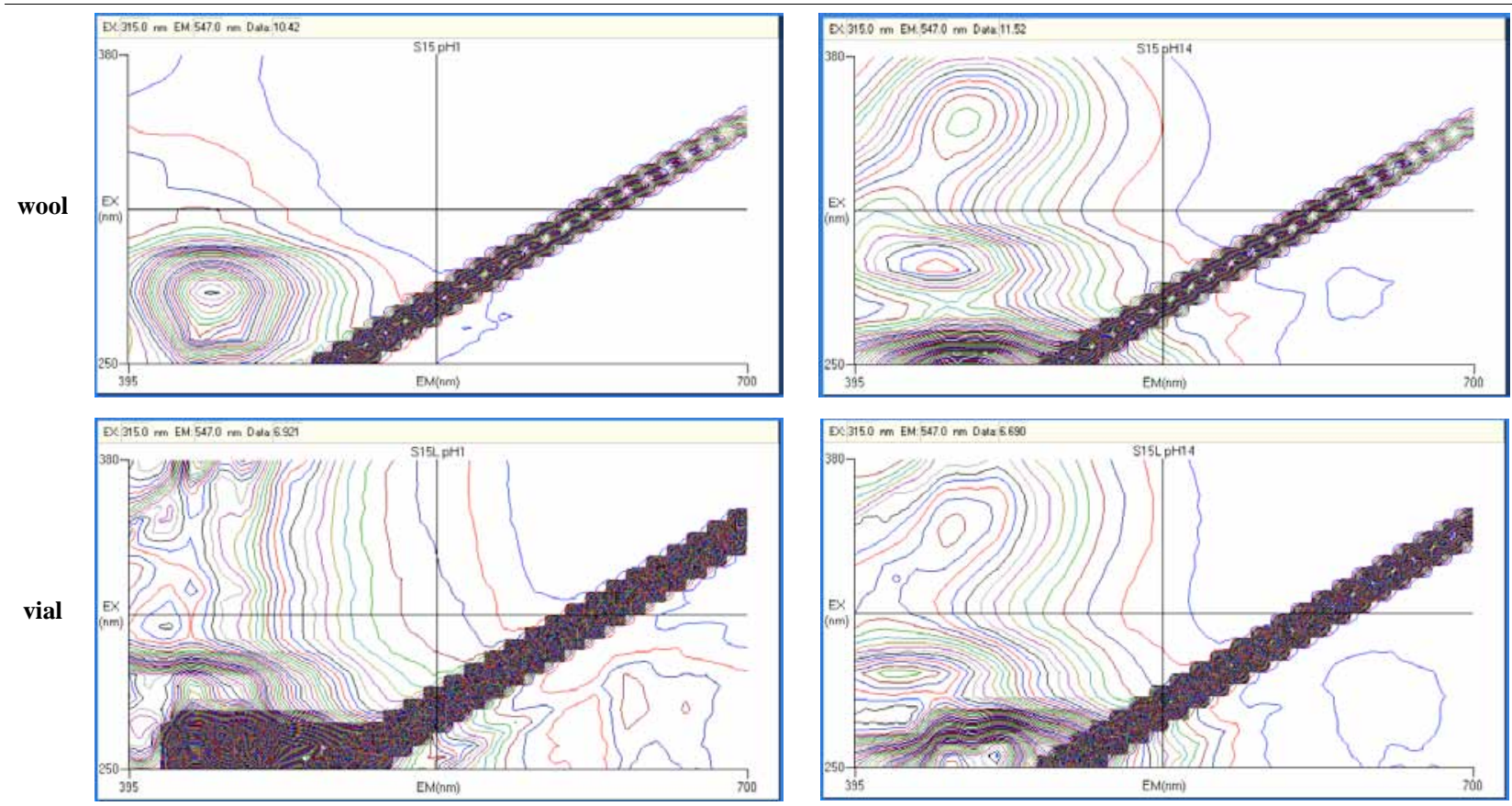

Sdb

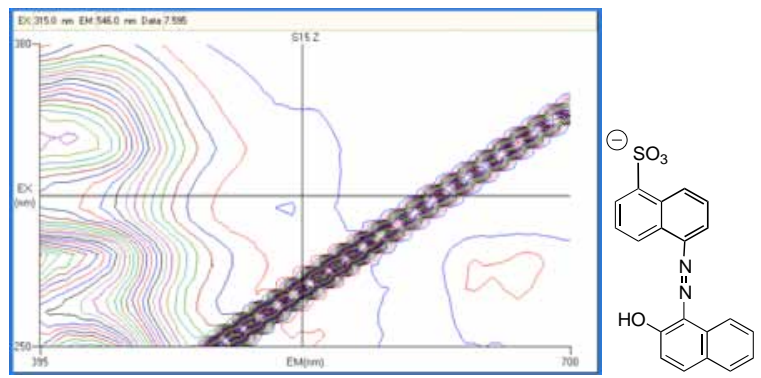




\section{Continued}
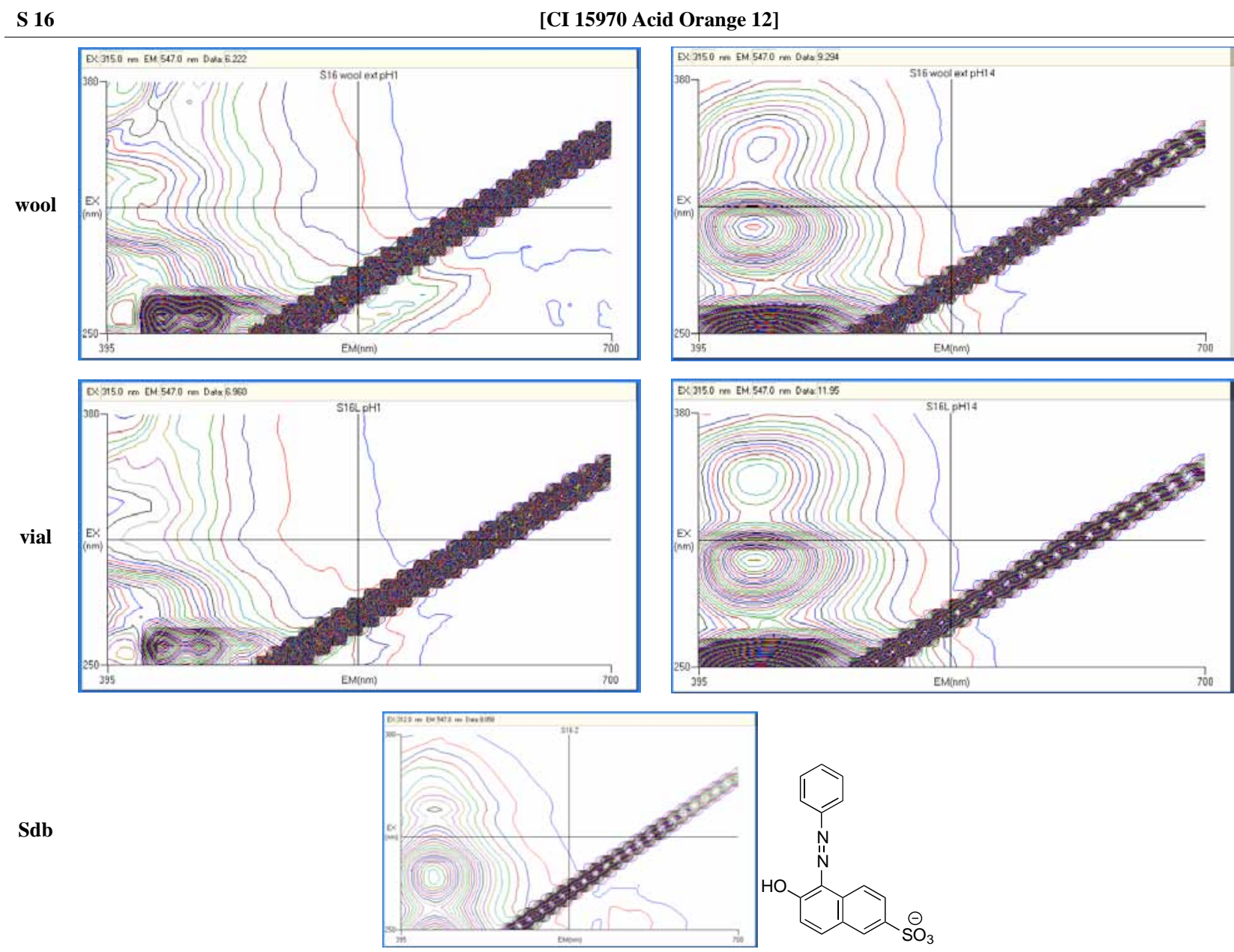

S 18

[CI 16050 Acid Red 25]
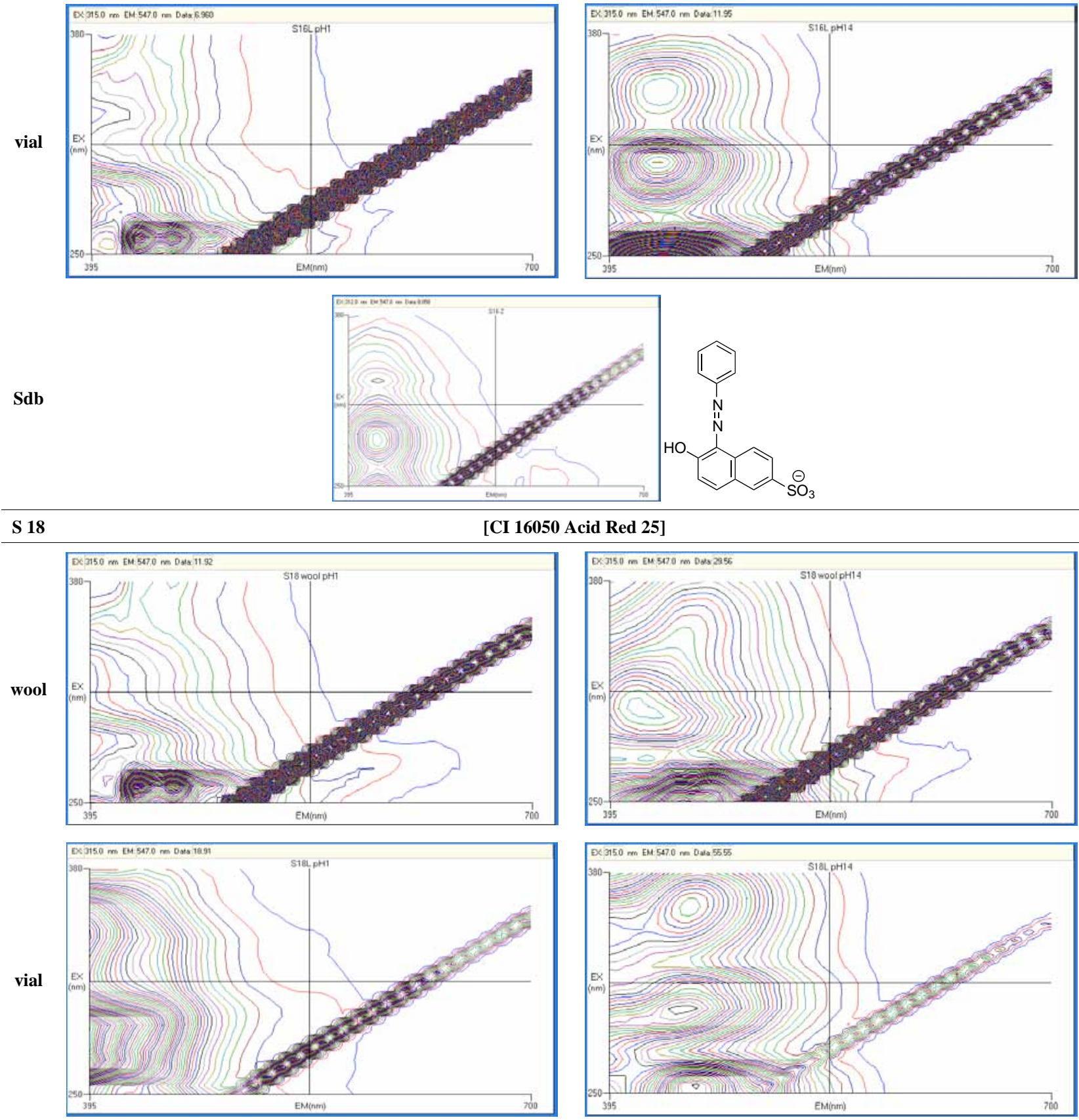

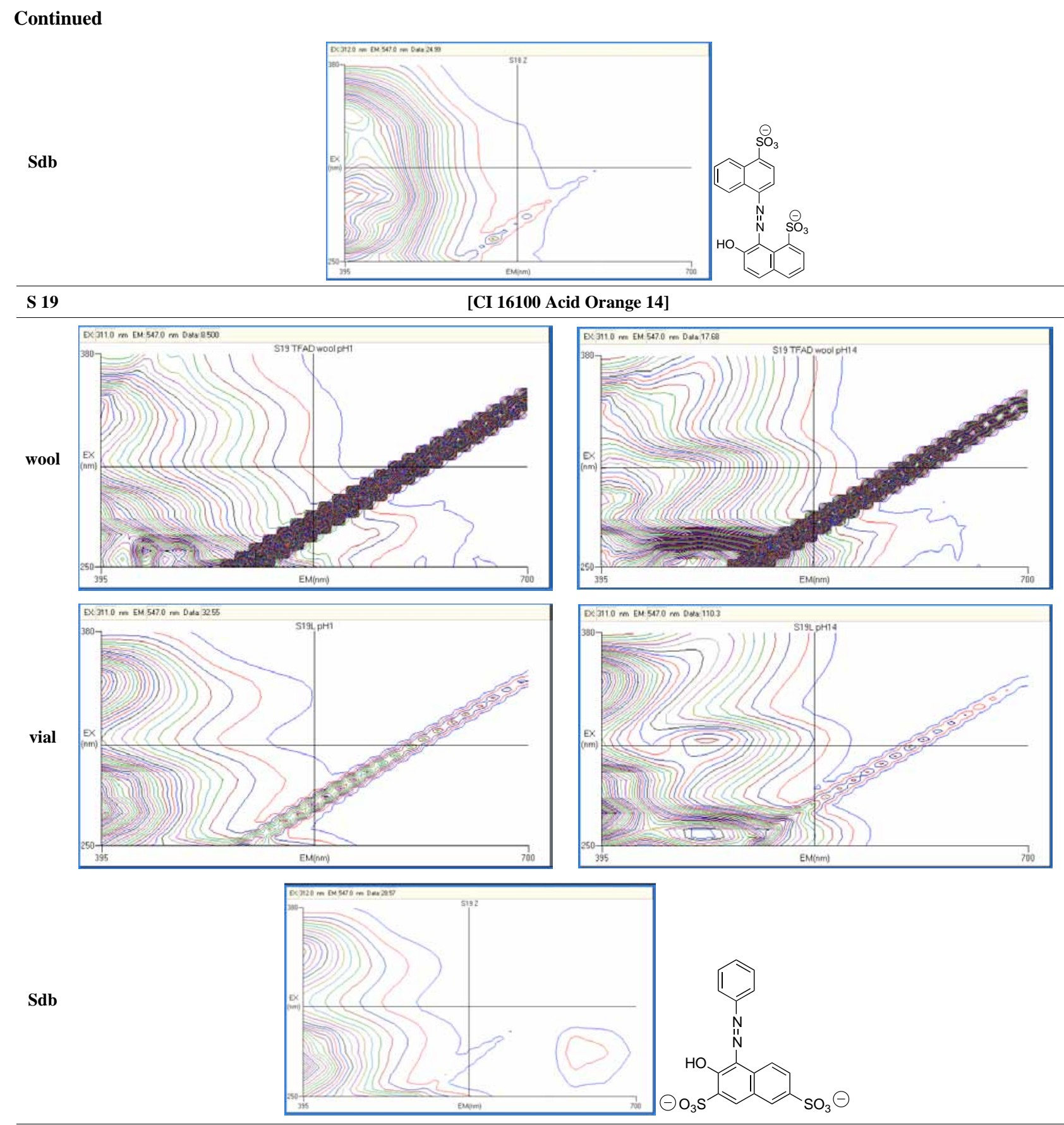

S 20

[CI 16150 Acid Red 26]
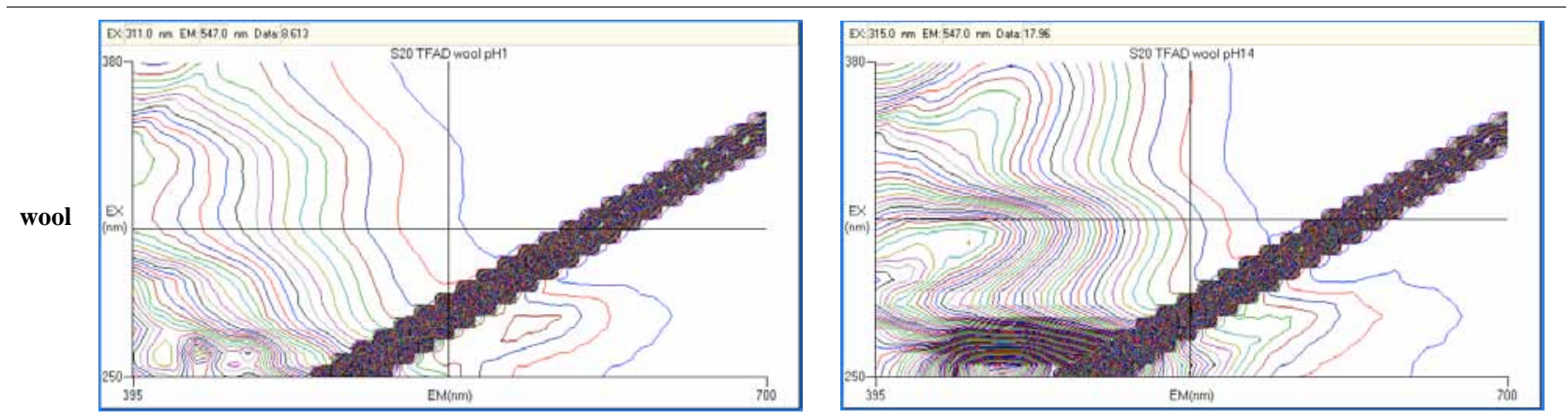


\section{Continued}
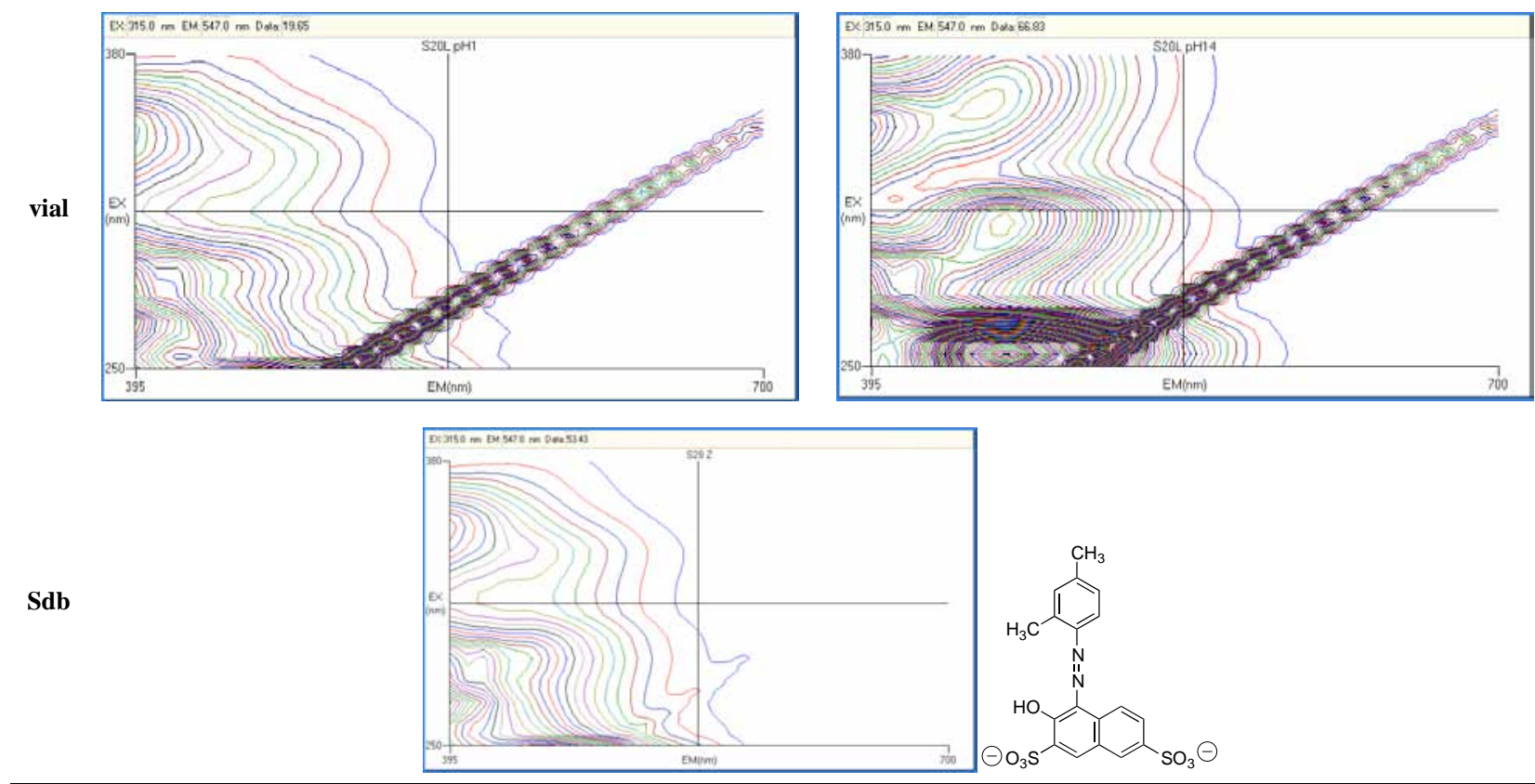

S $21 \quad$ [CI 16180 Acid Red 17]
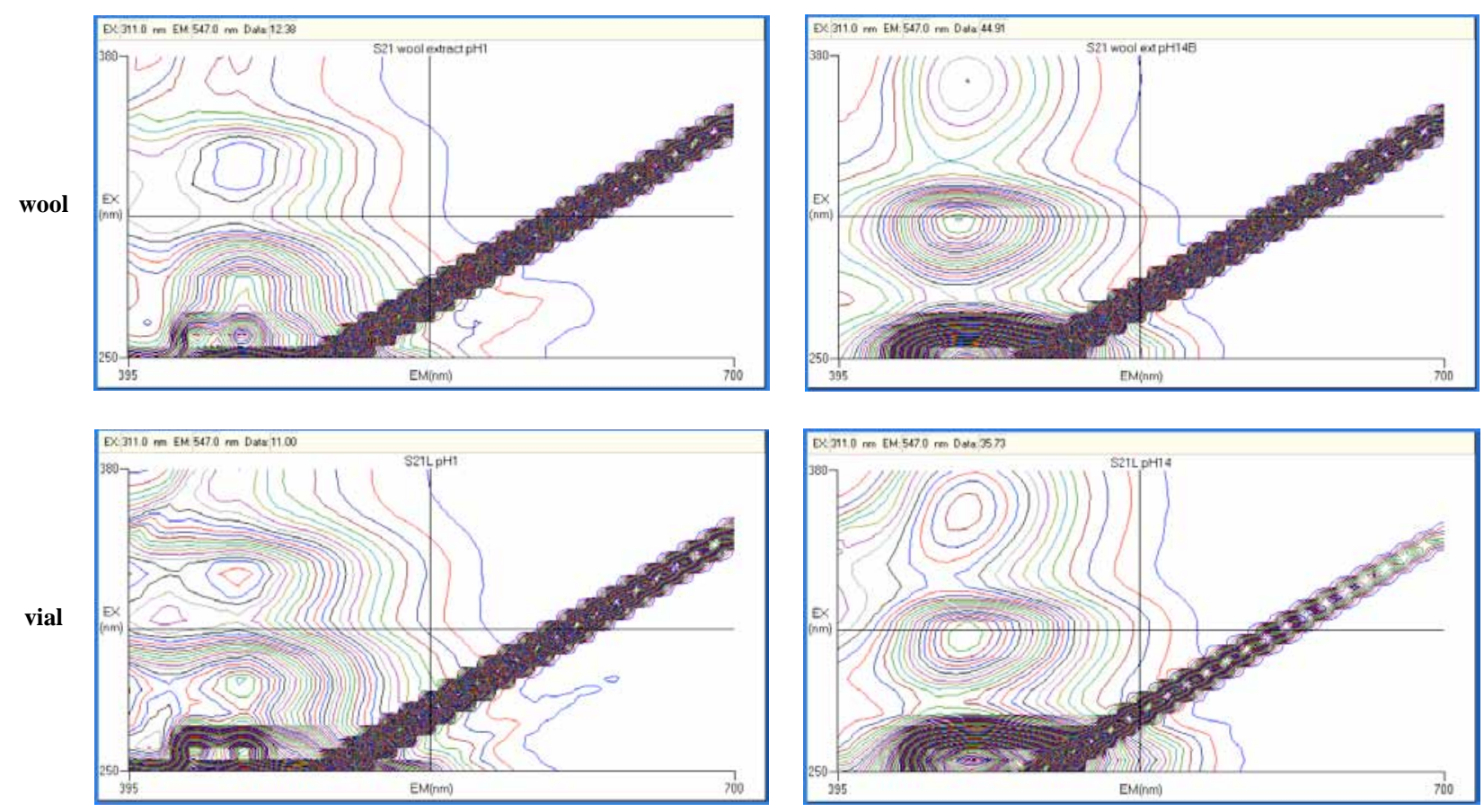

Sdb
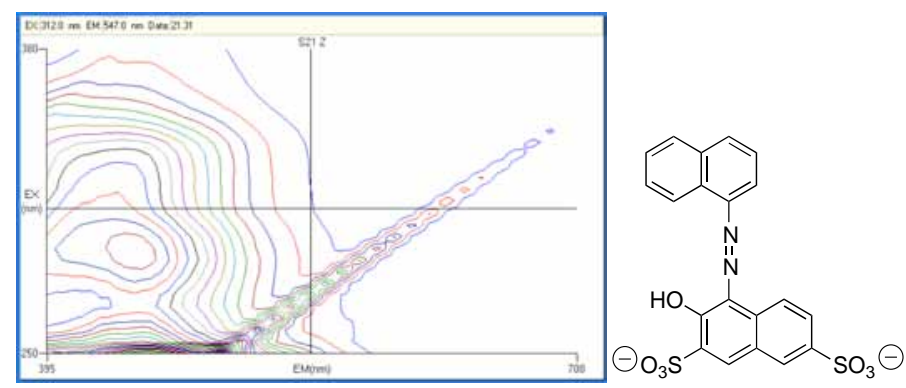


\section{Continued}
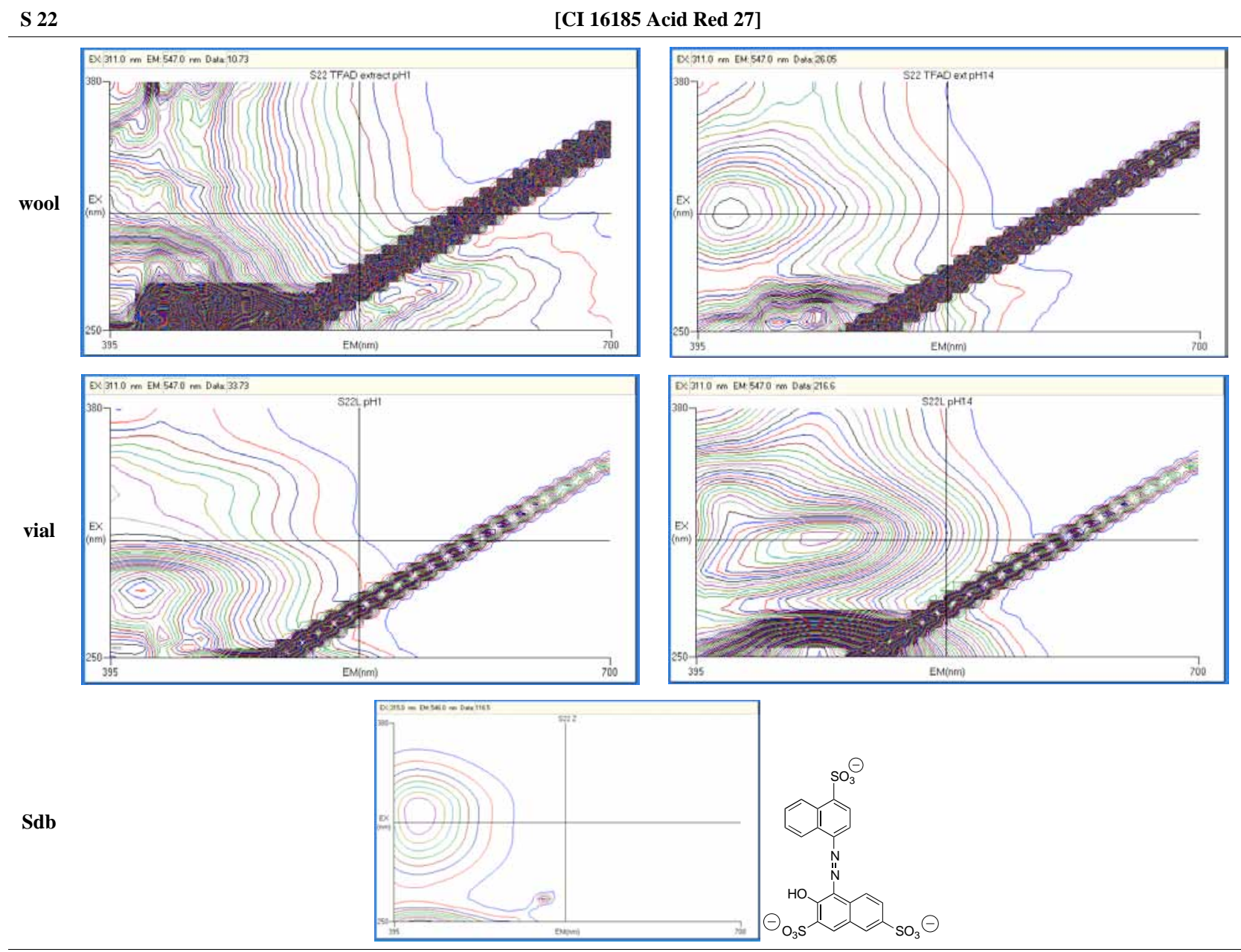

[CI 16230 Acid Orange 10]
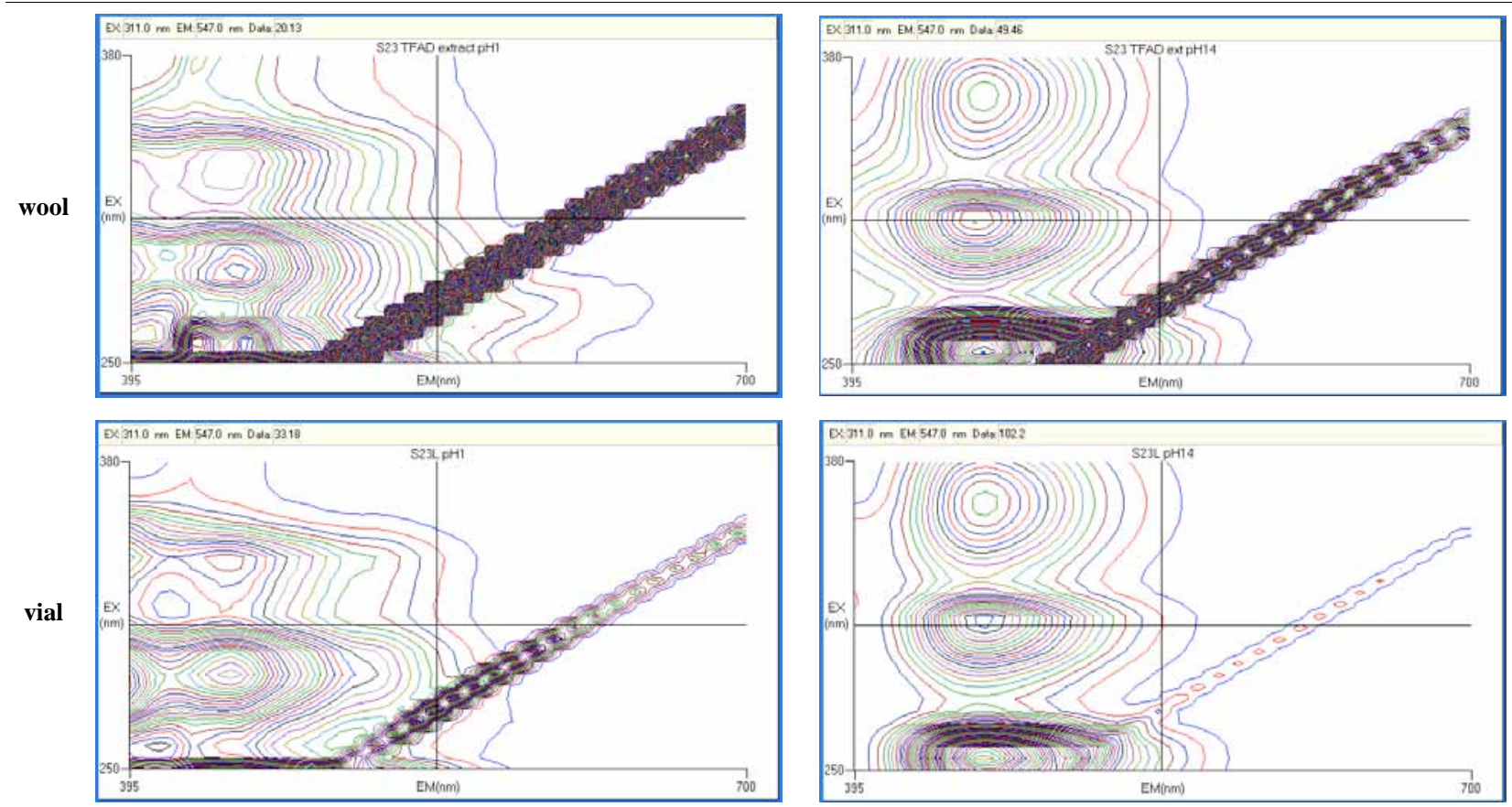


\section{Continued}

Sdb
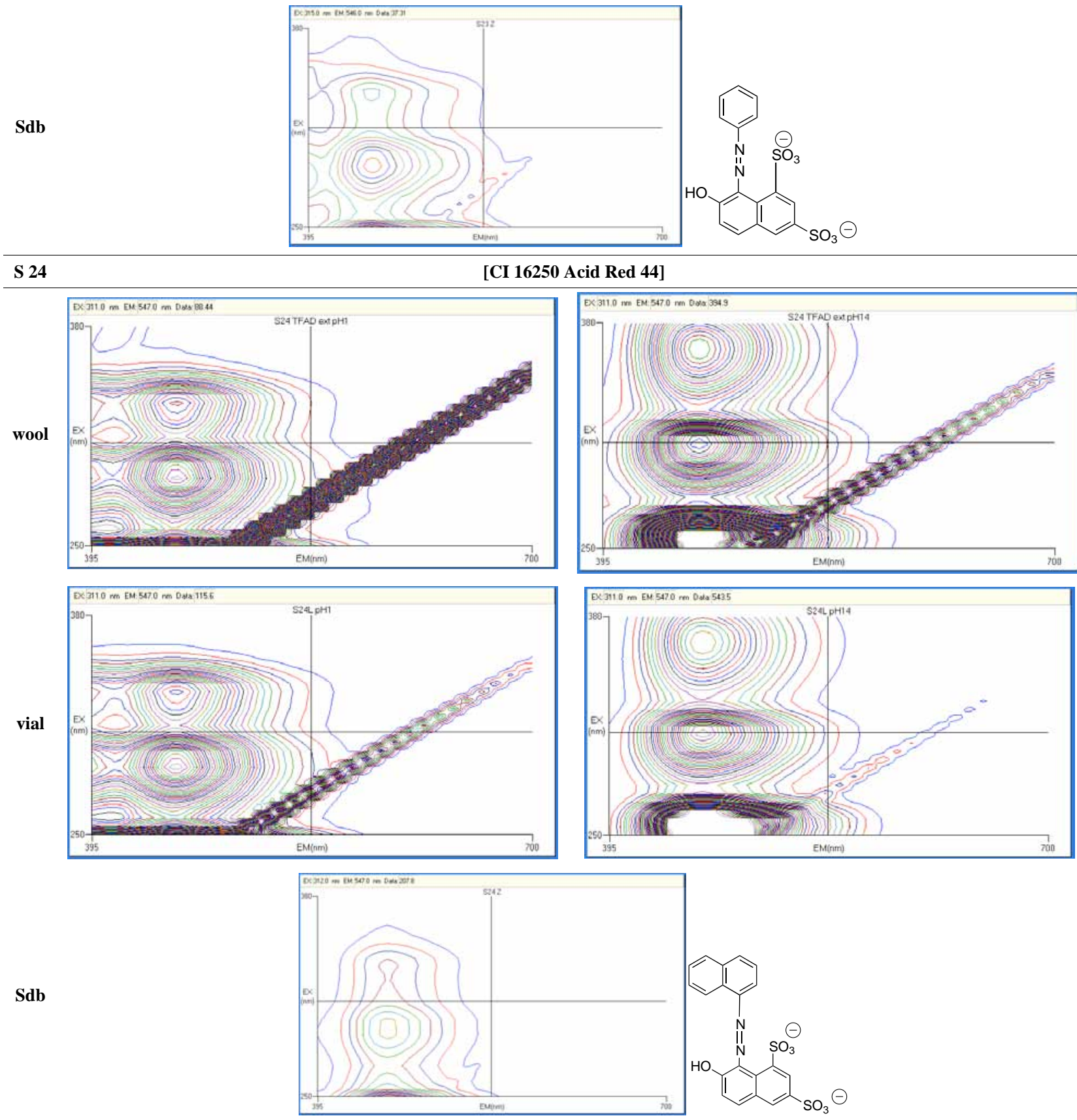

Sdb

[CI 16290 Acid Red 41]

S 26
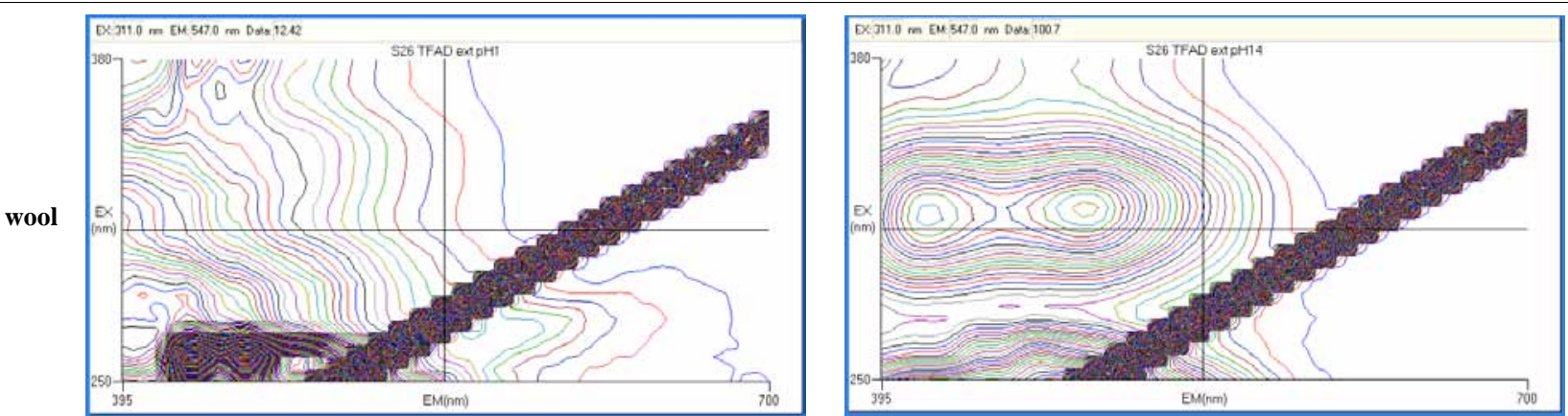


\section{Continued}
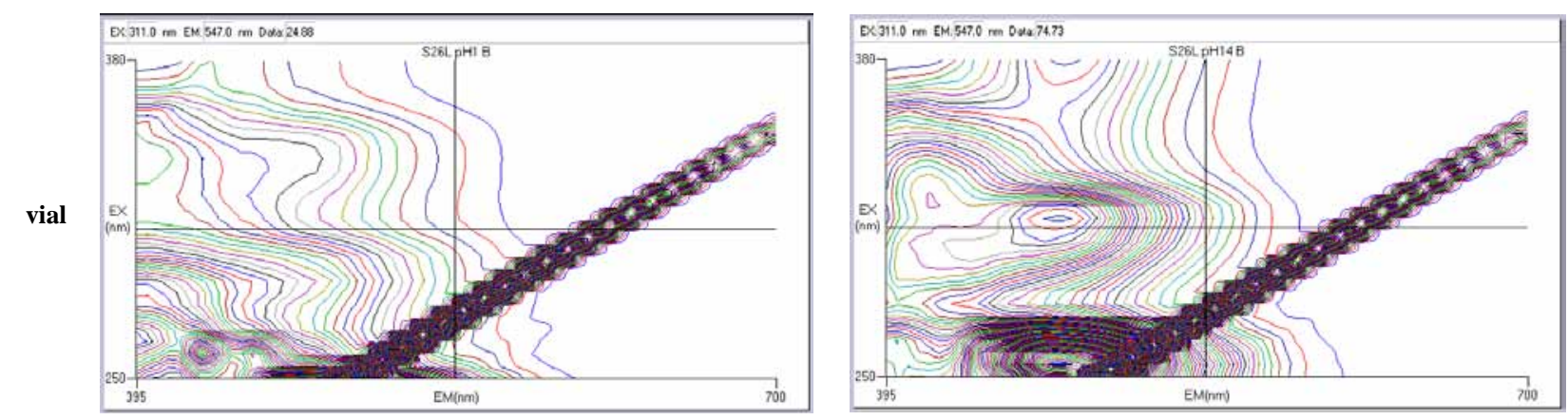

Sdb

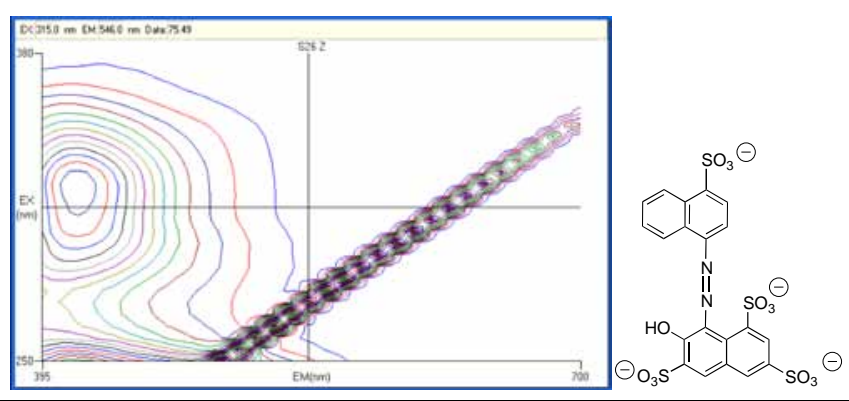

S 27

[CI 17200 Acid Red 33]
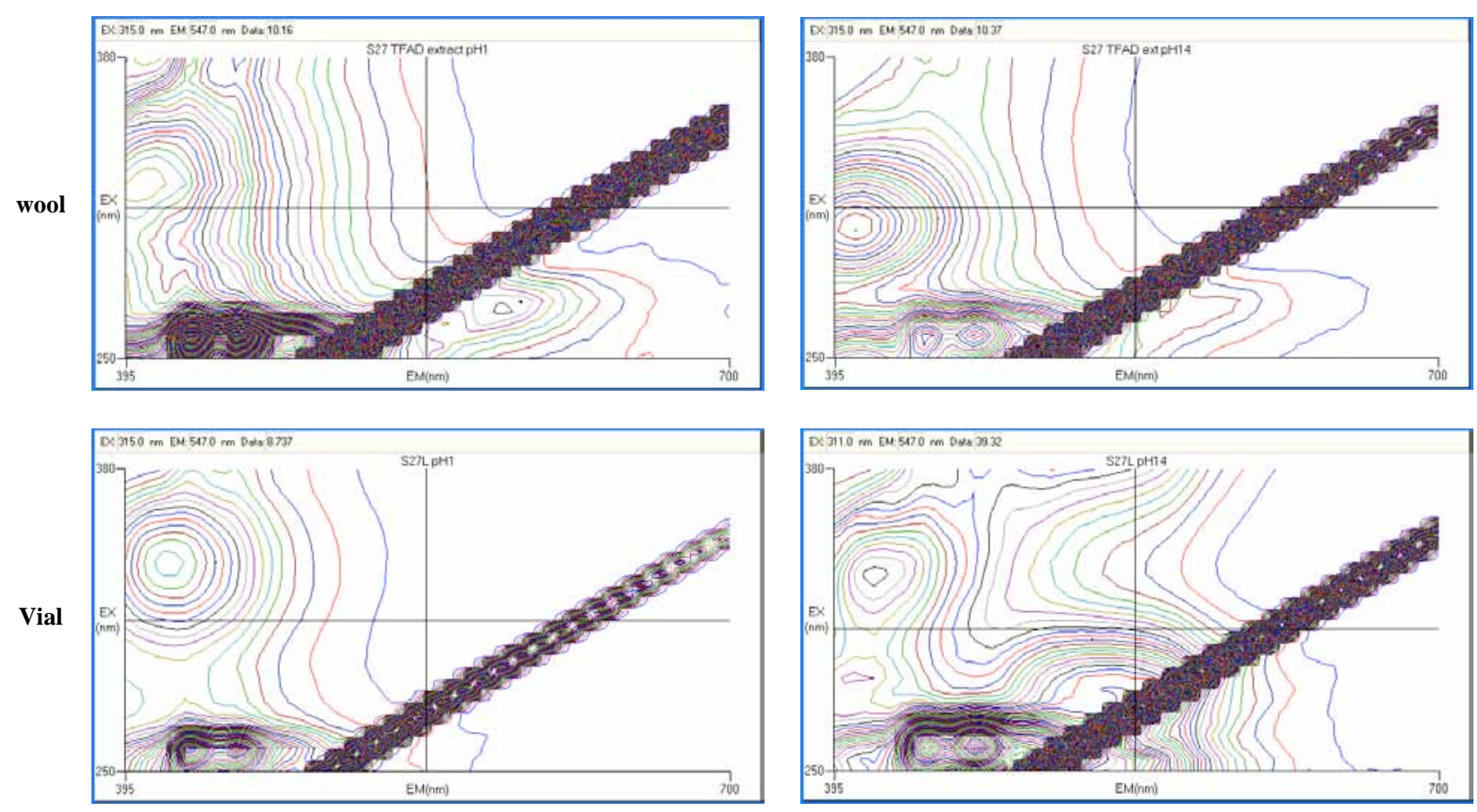

Sdb
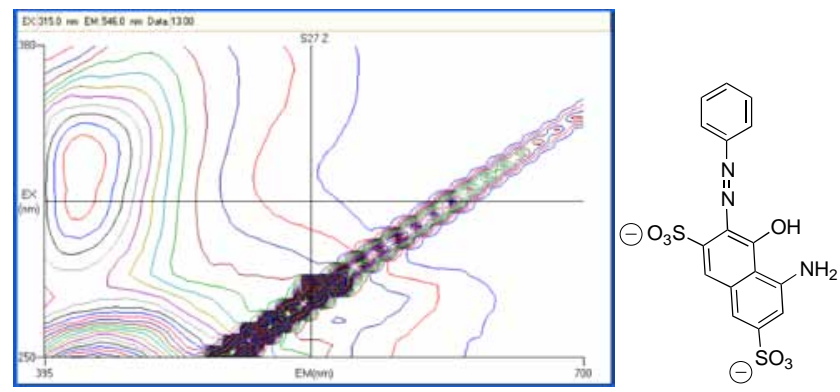


\section{Continued}
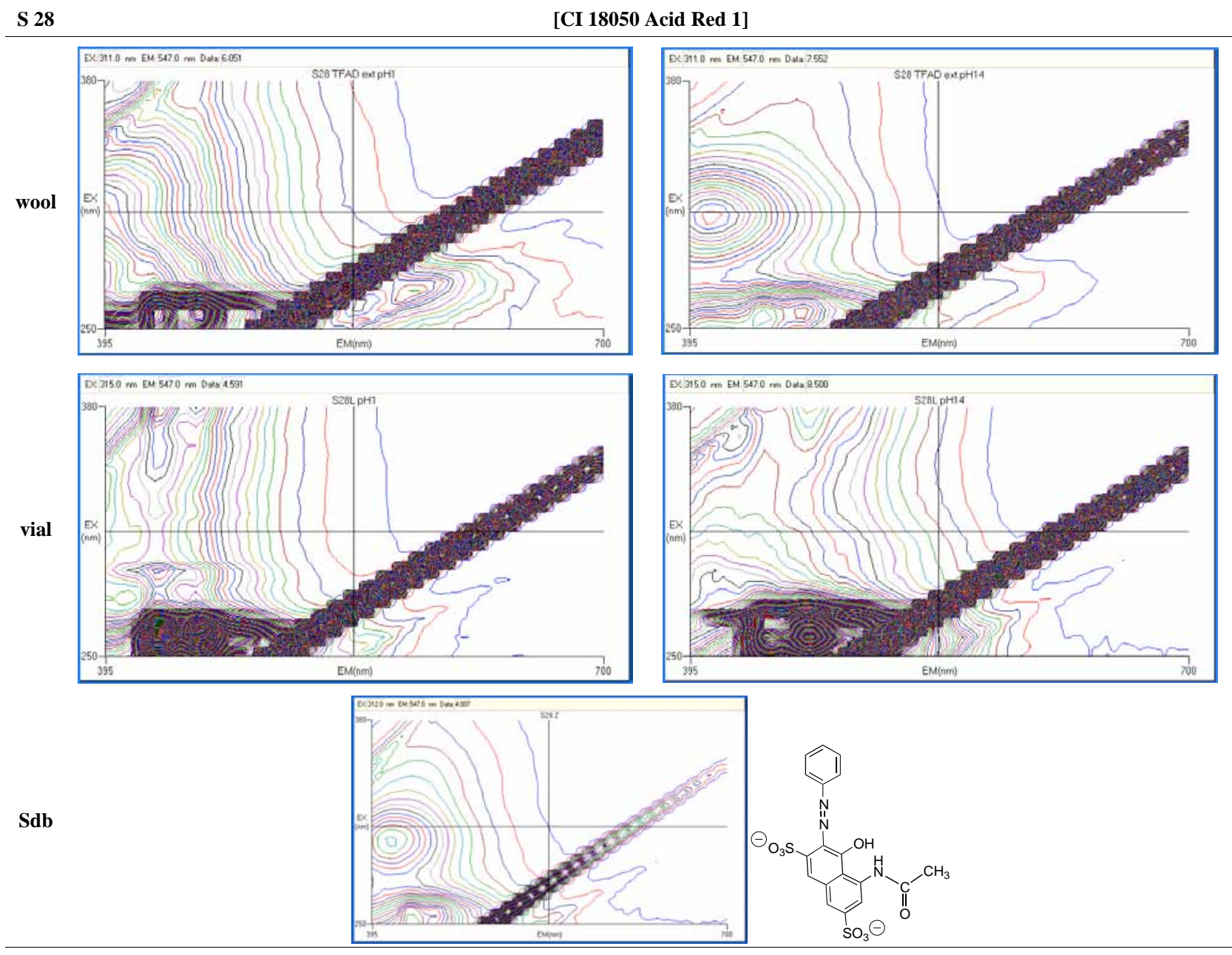

S 30

[CI 18820 Acid Yellow 11]
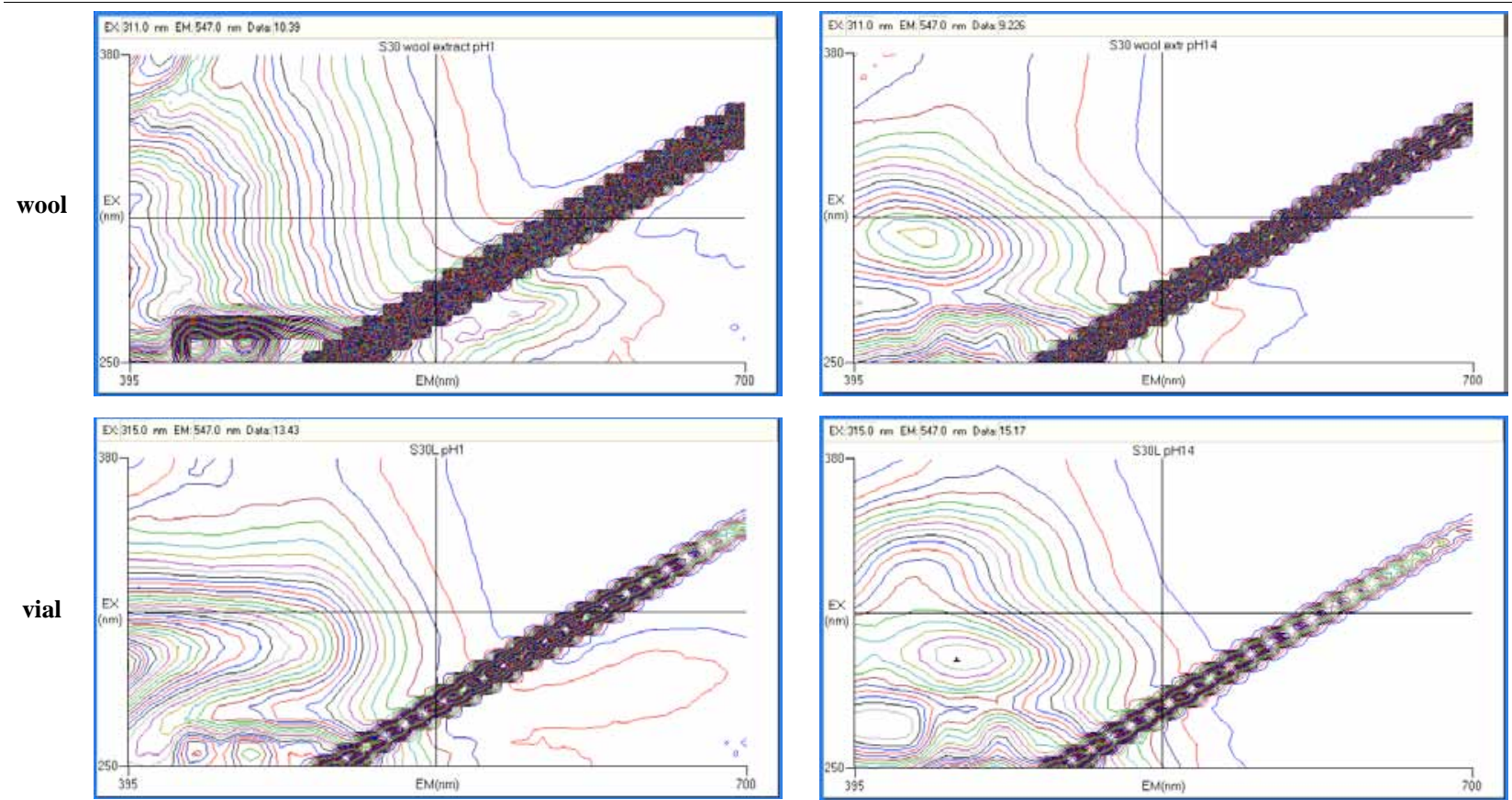


\section{Continued}

Sdb

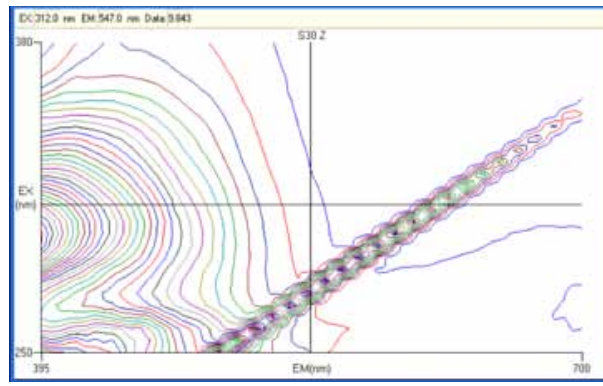

(c)
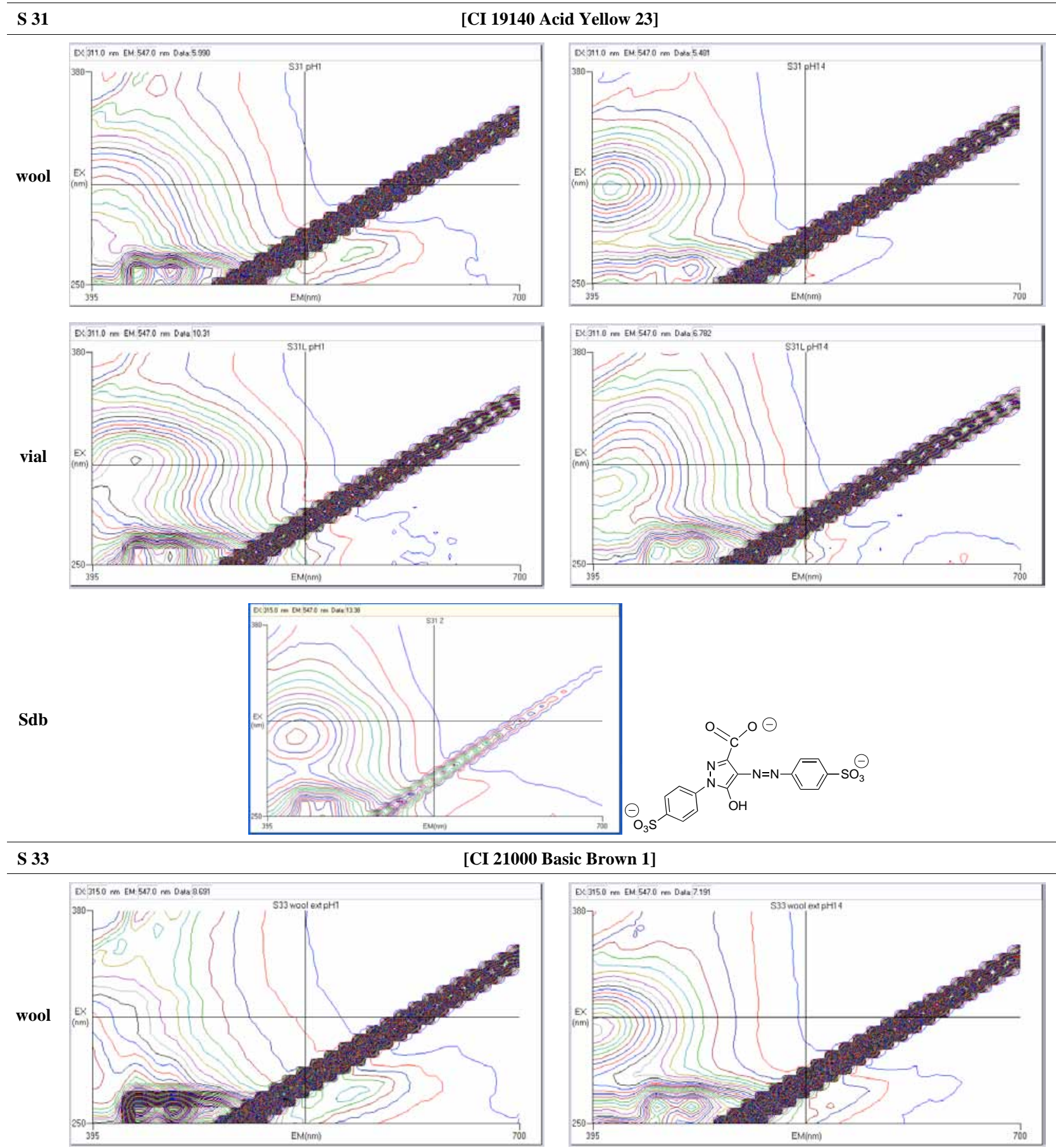


\section{Continued}
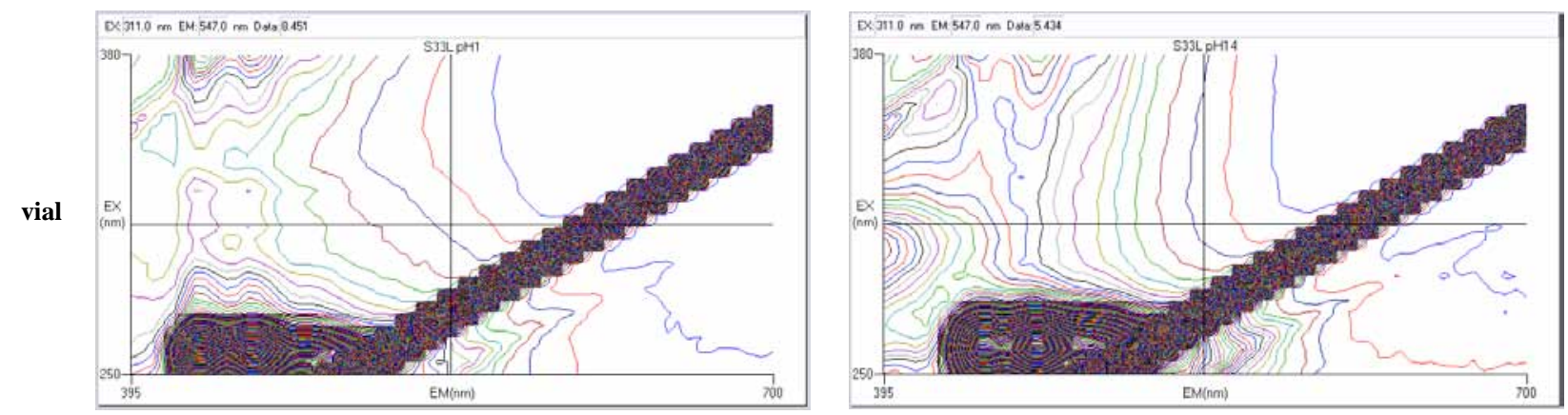

Sdb

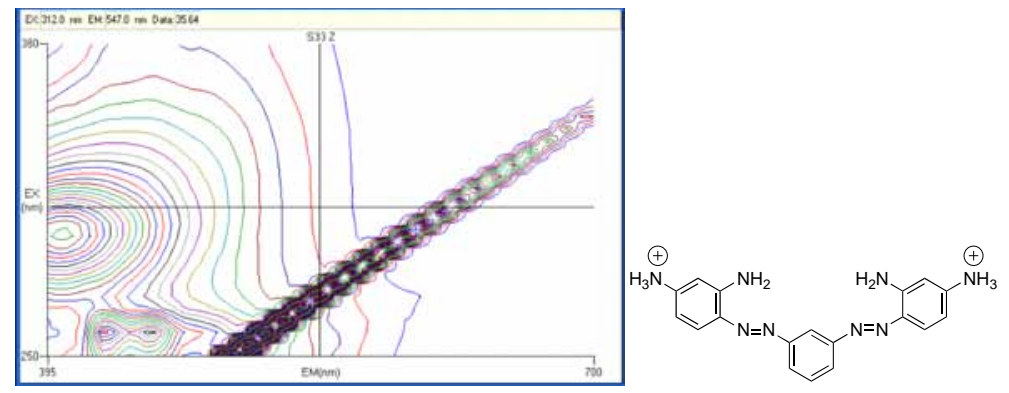

S 34

[CI 22120 Direct Red 28]
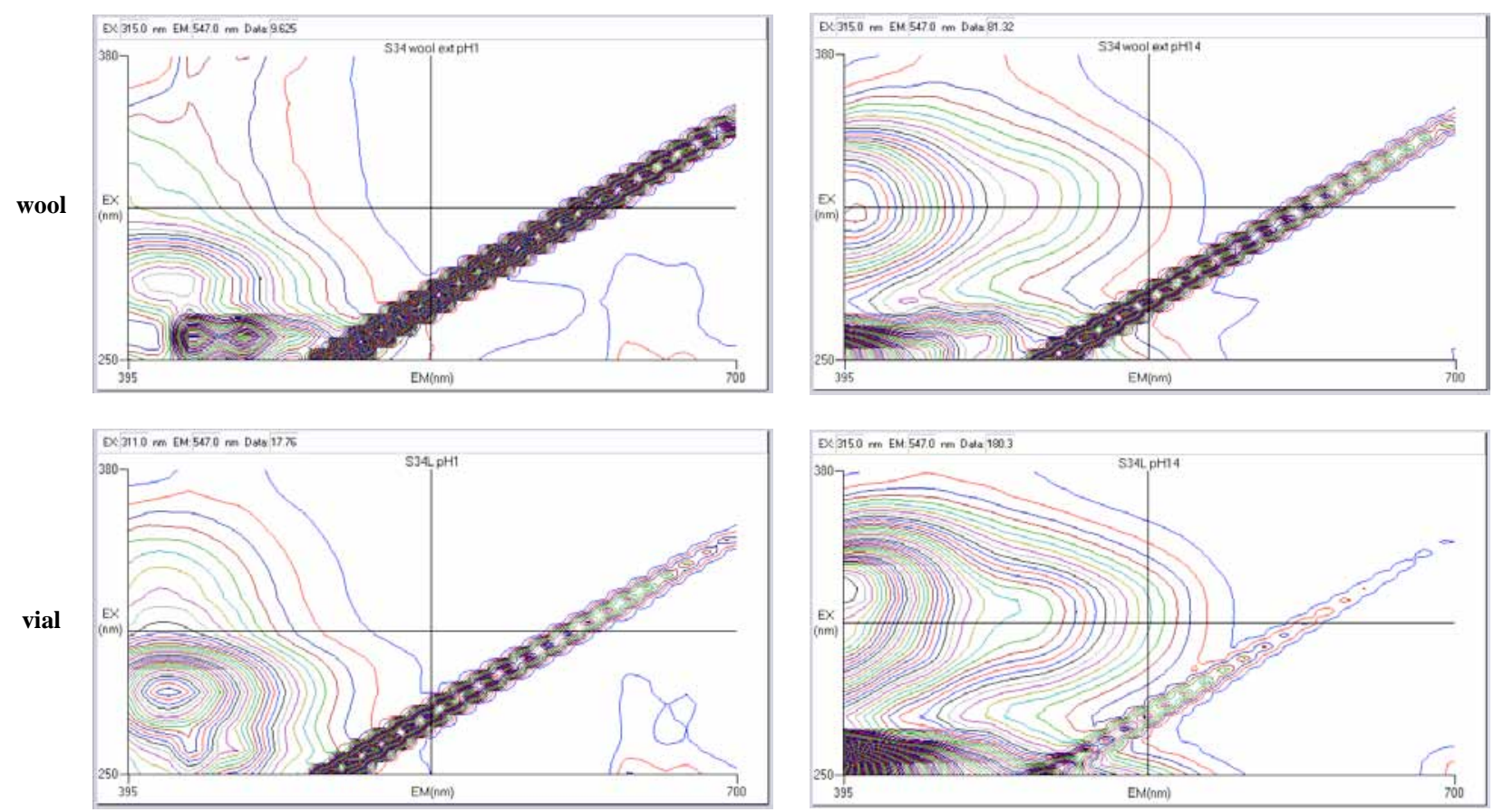

Sdb

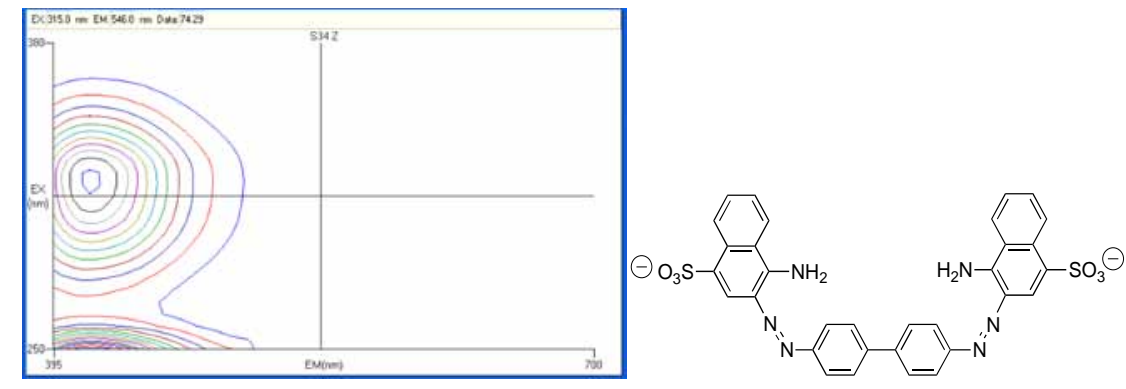




\section{Continued}

S 35

[CI 24890 Direct Yellow 4]
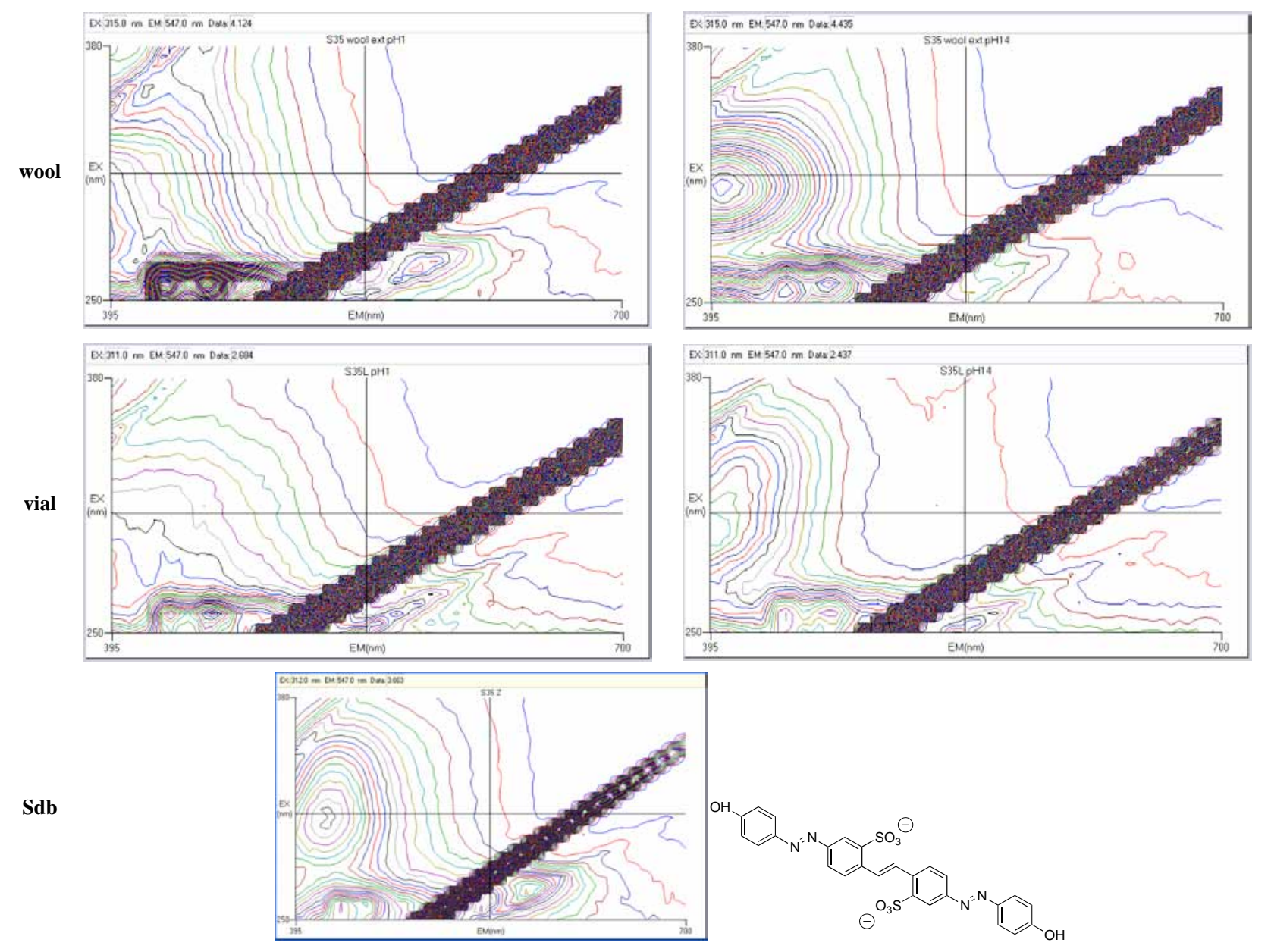

S 36

[CI 26900 Acid Red 151]
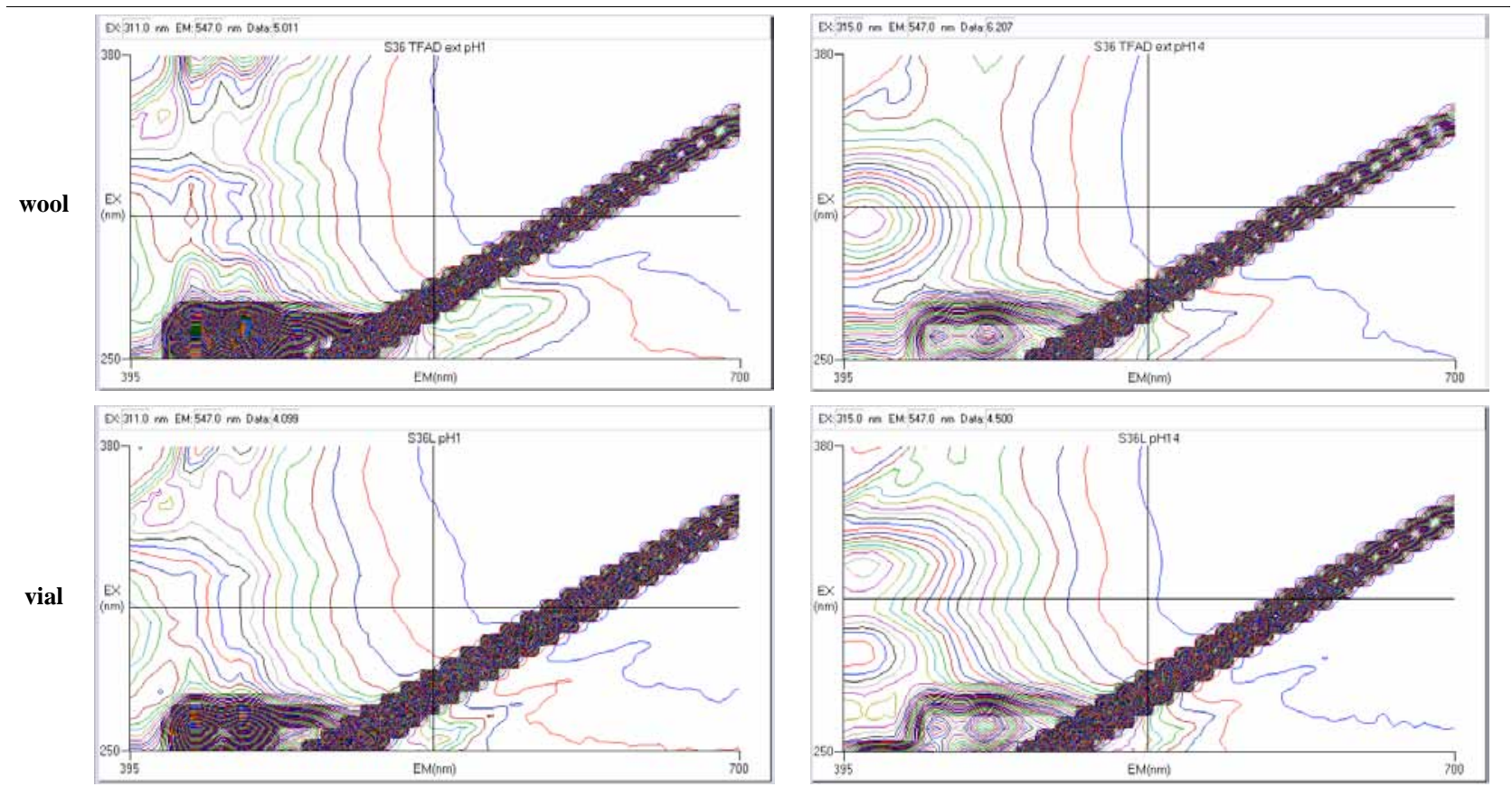


\section{Continued}

Sdb

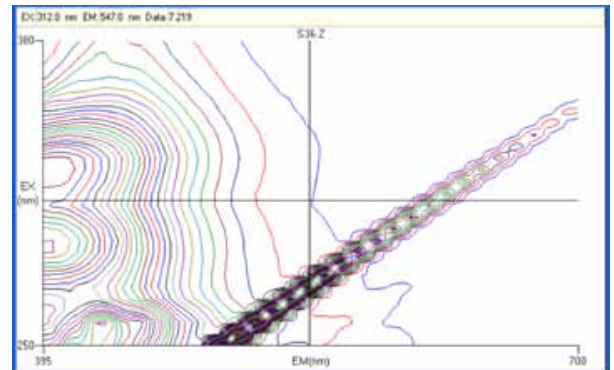

$\Theta \mathrm{O}_{3}-\left\langle-\mathrm{N}_{\mathrm{HO}}\right.$

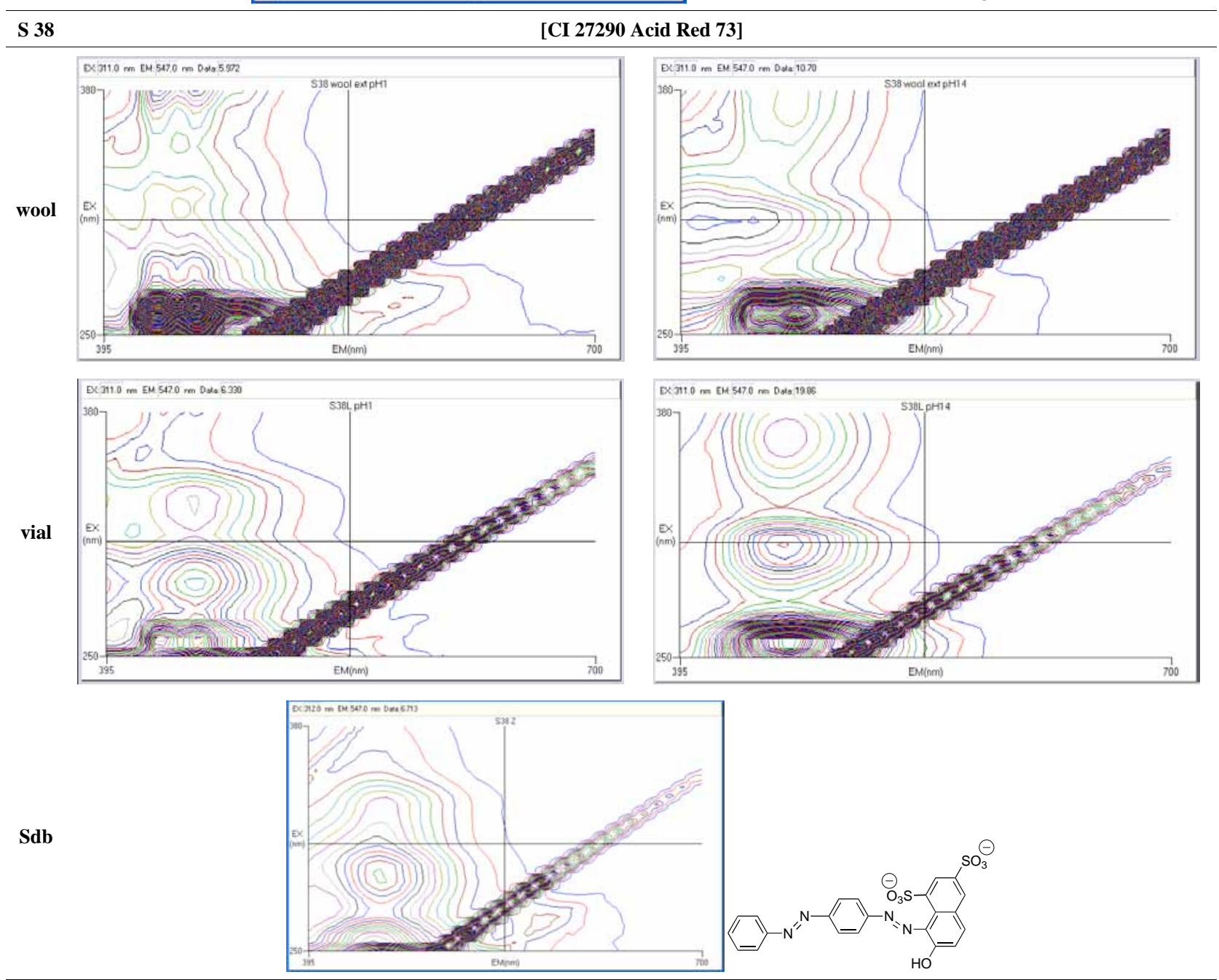

S 41

[CI 42040 Basic Green 1]
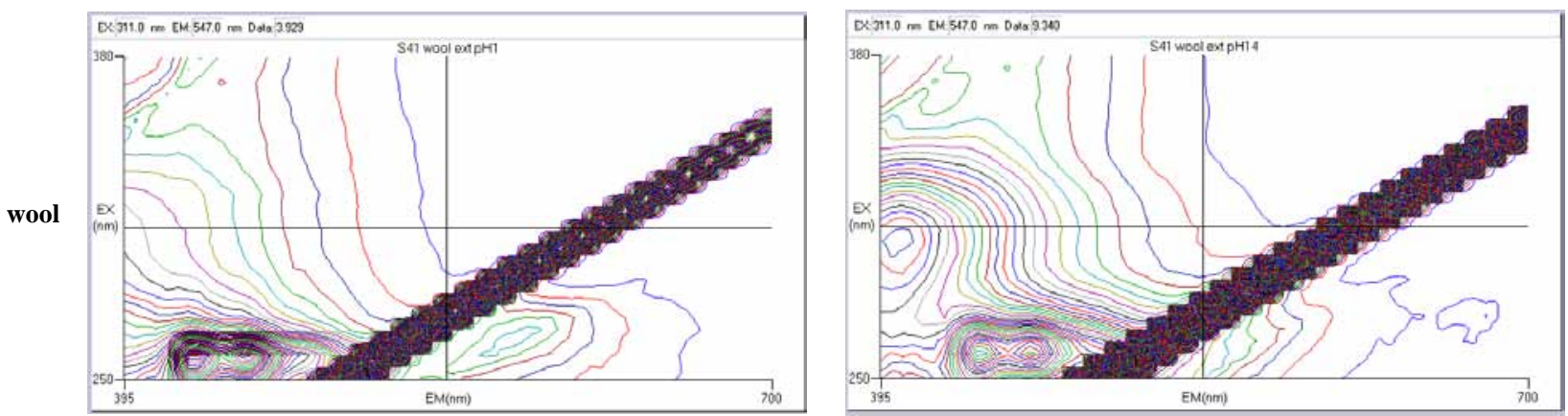


\section{Continued}
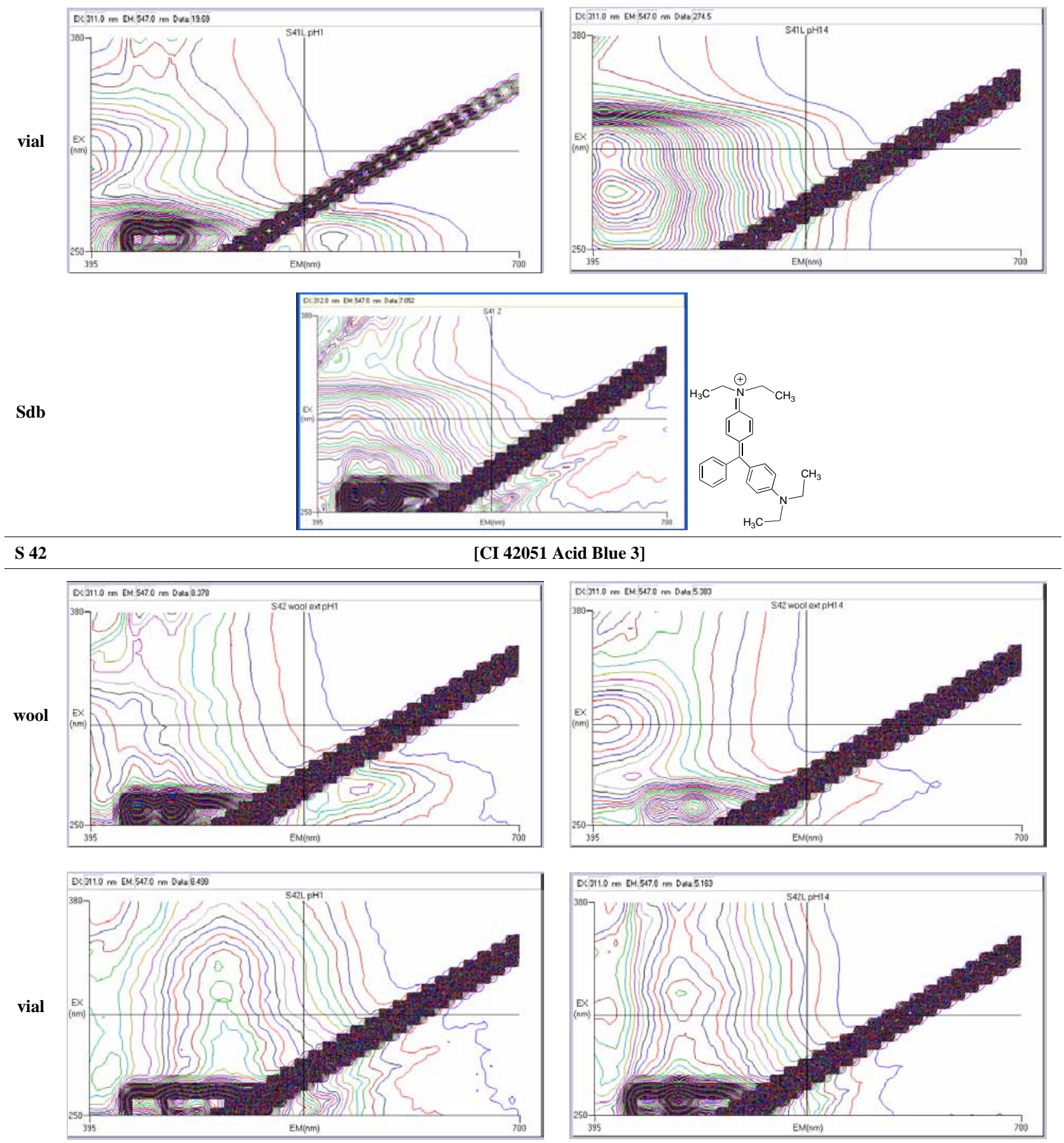

Sdb

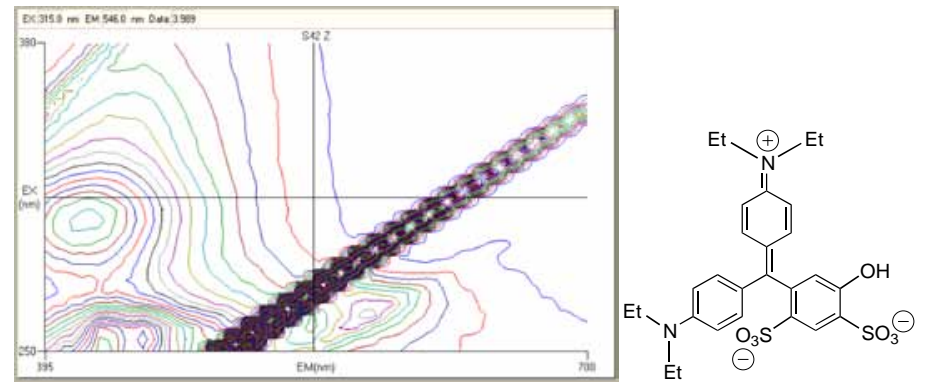




\section{Continued}
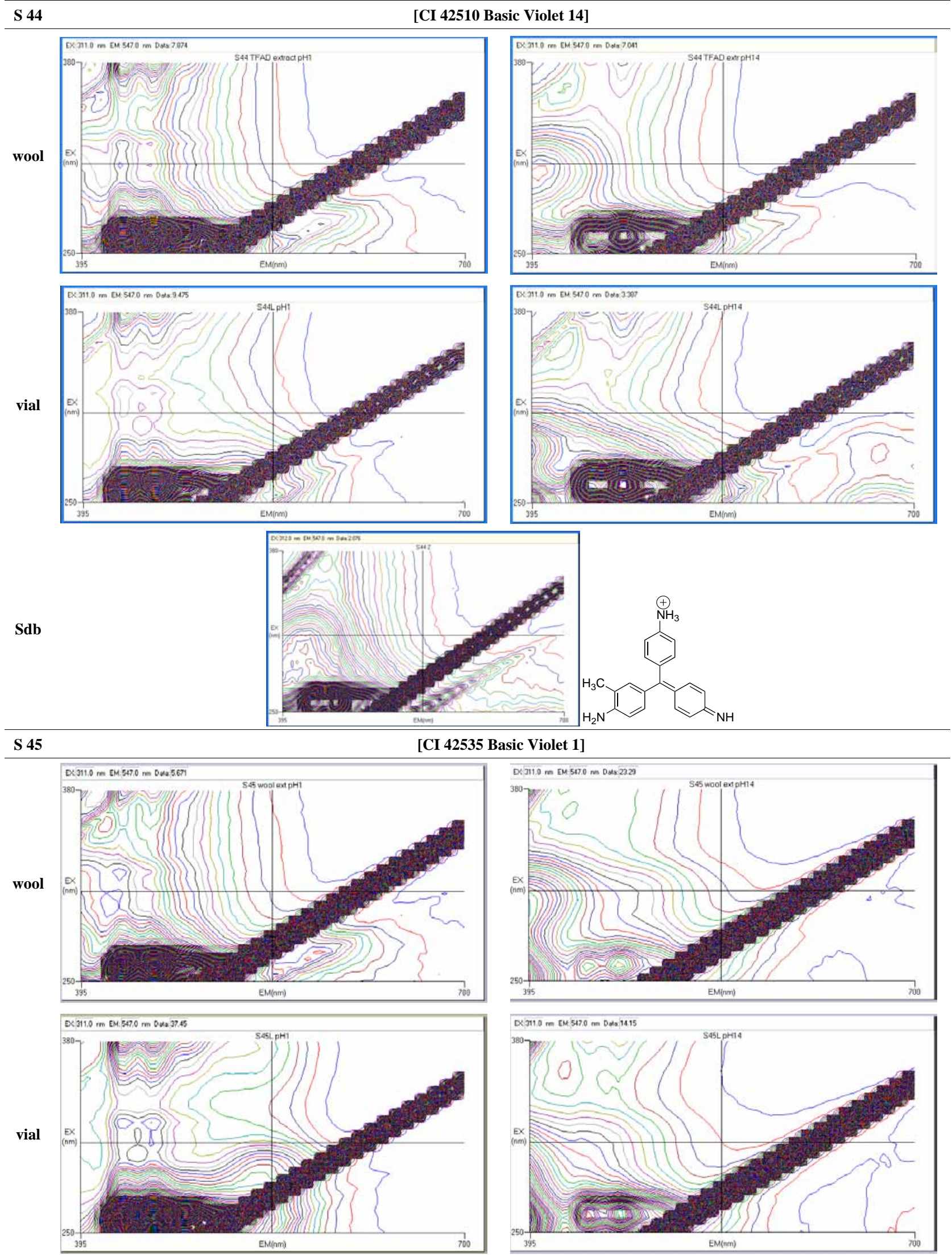


\section{Continued}

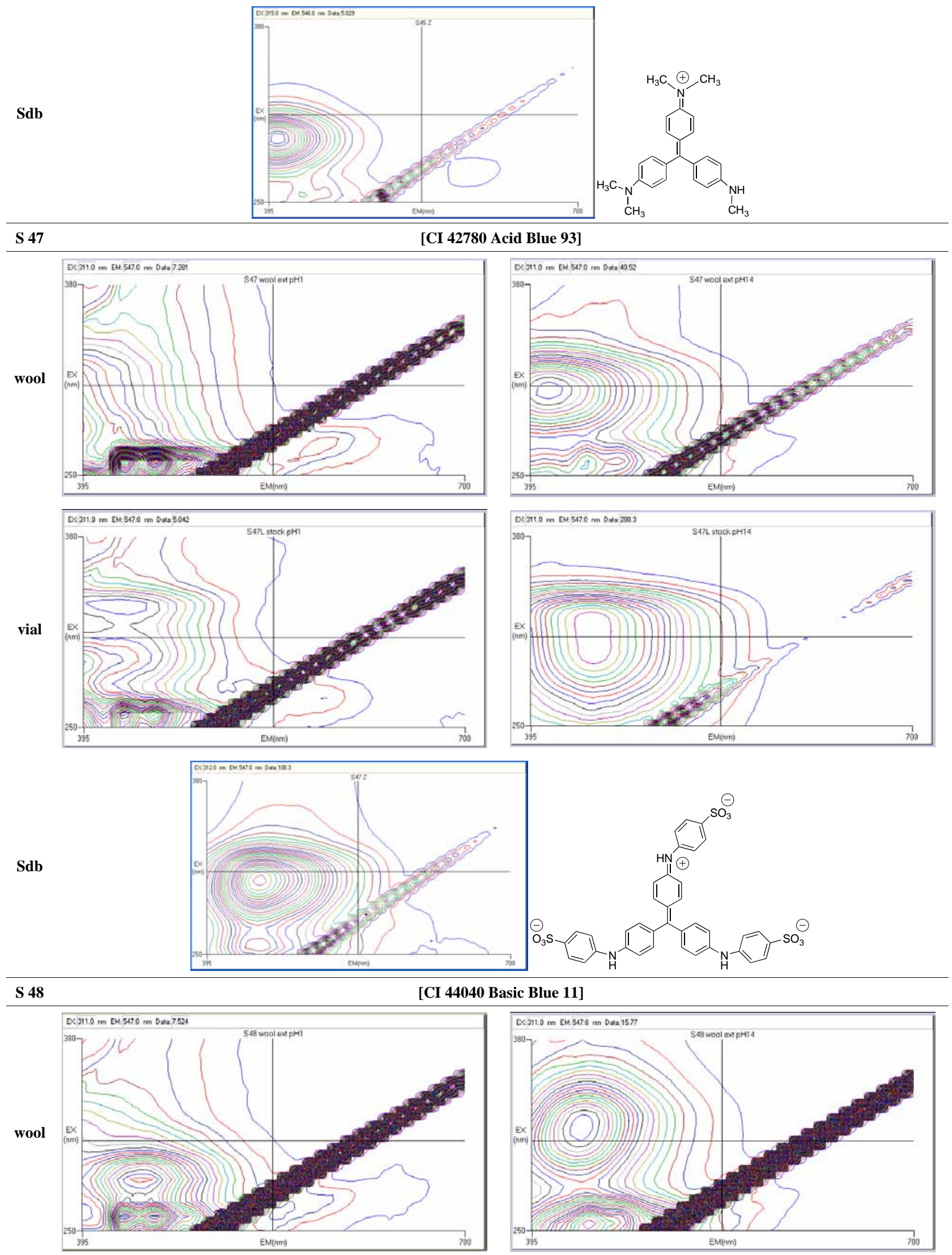




\section{Continued}
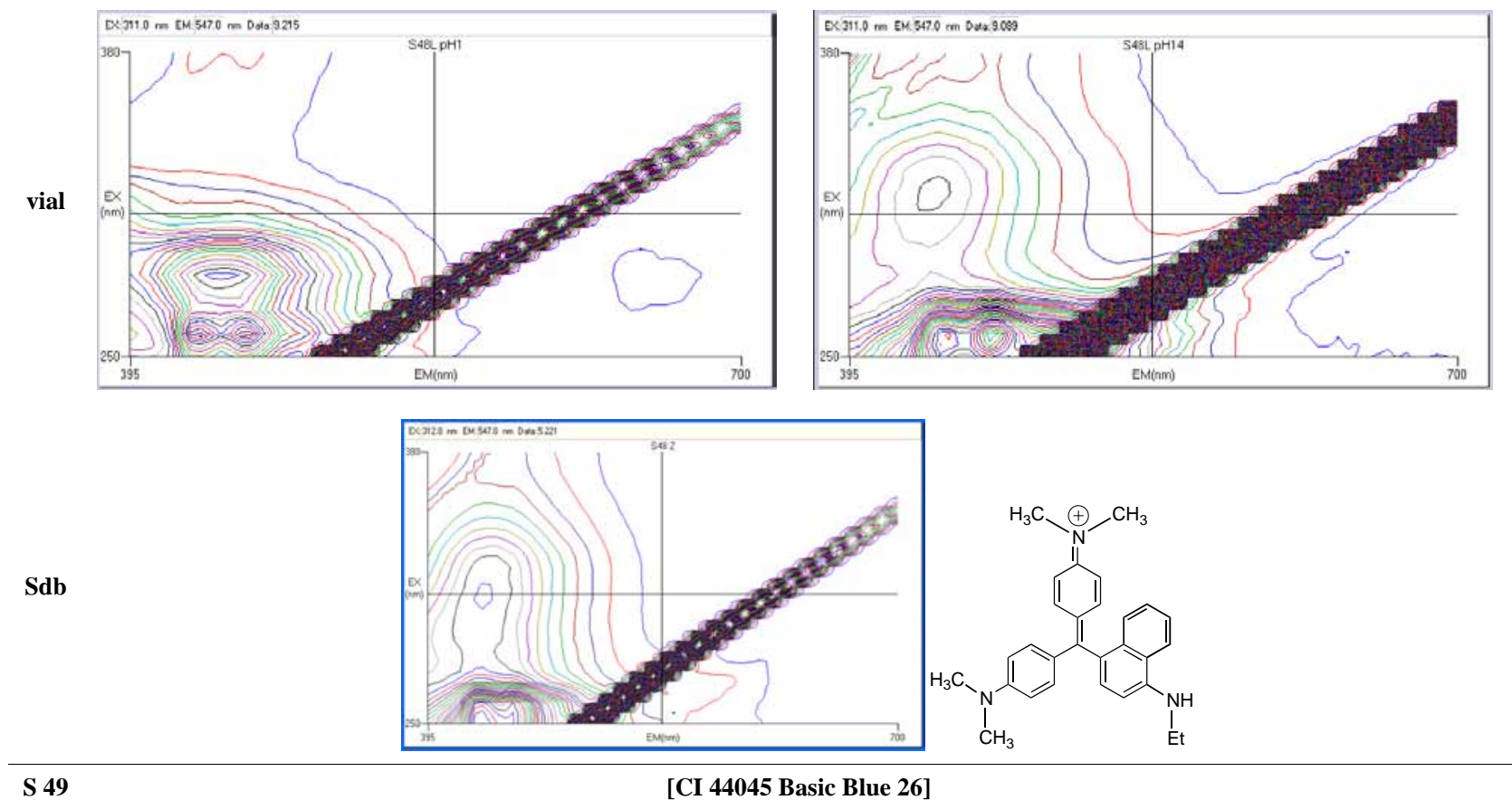

S 49

[CI 44045 Basic Blue 26]
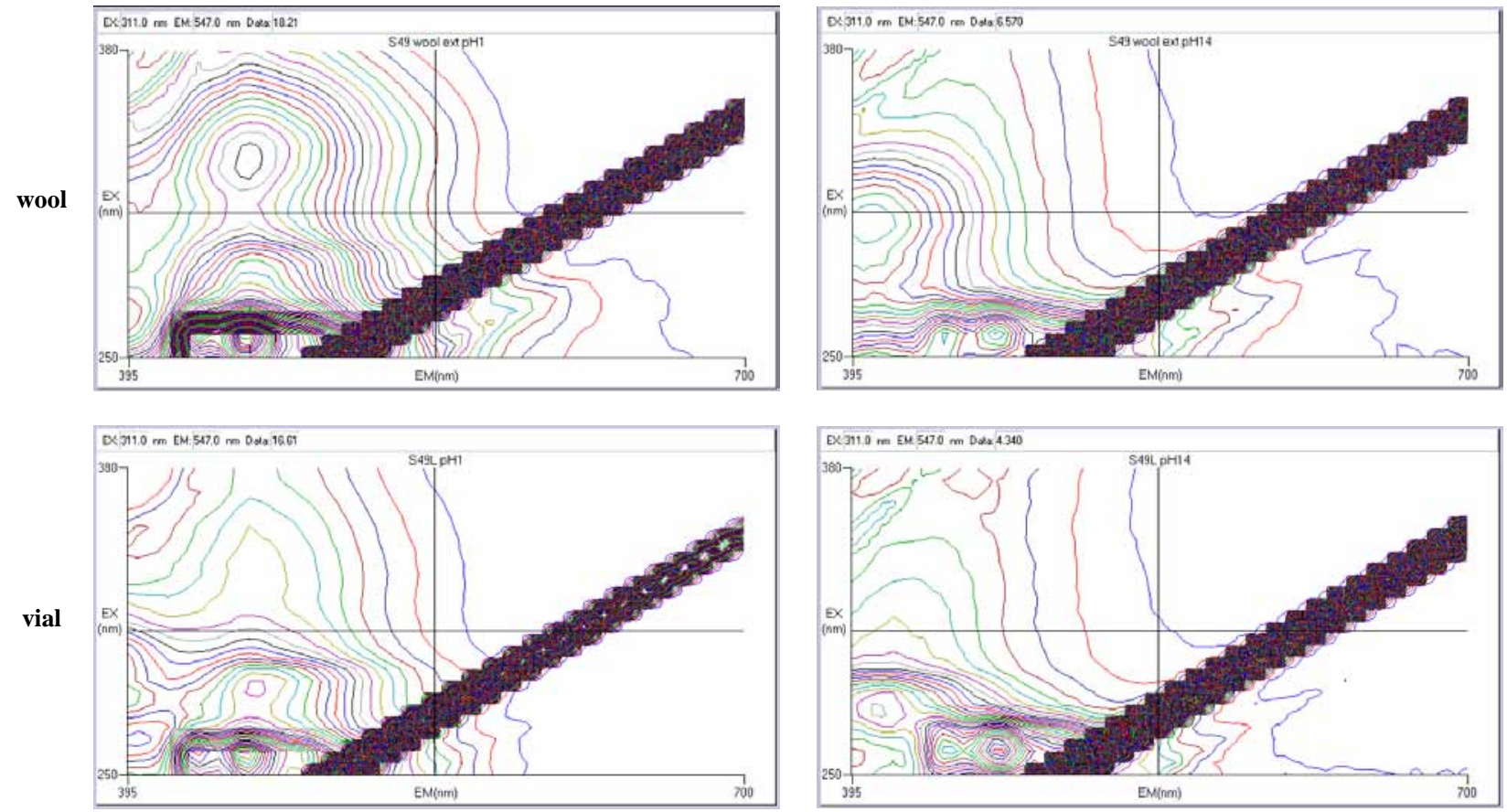

Sdb

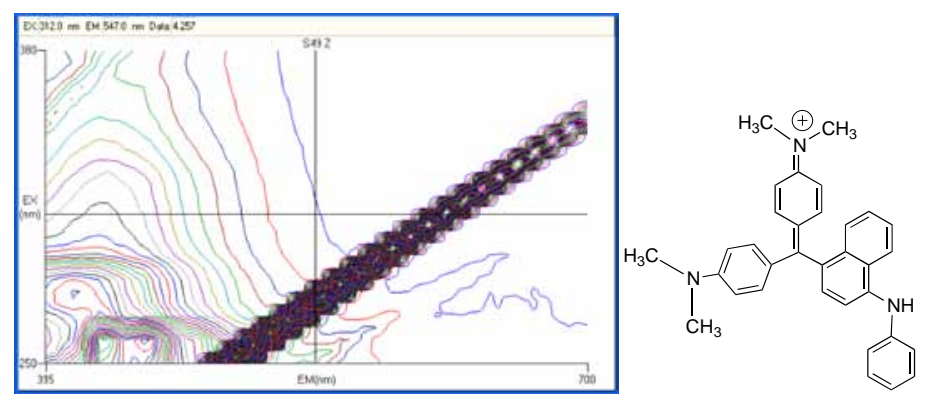




\section{Continued}

S 50
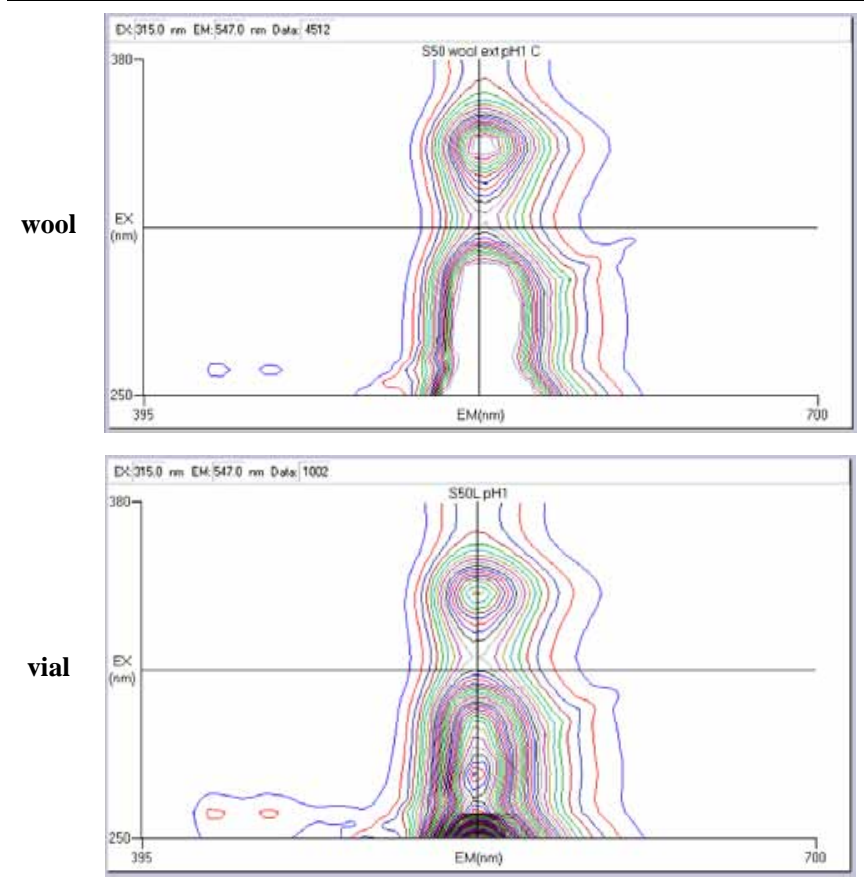

EM(nm)

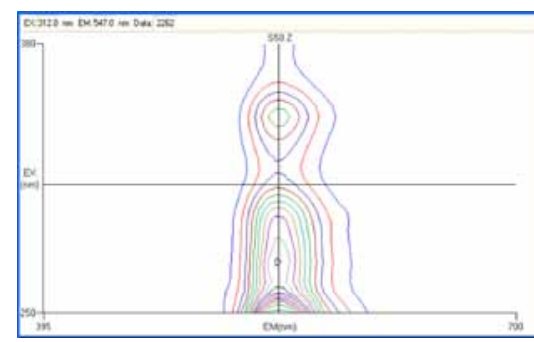

Sdb

[CI 45170 Solvent Red 49]

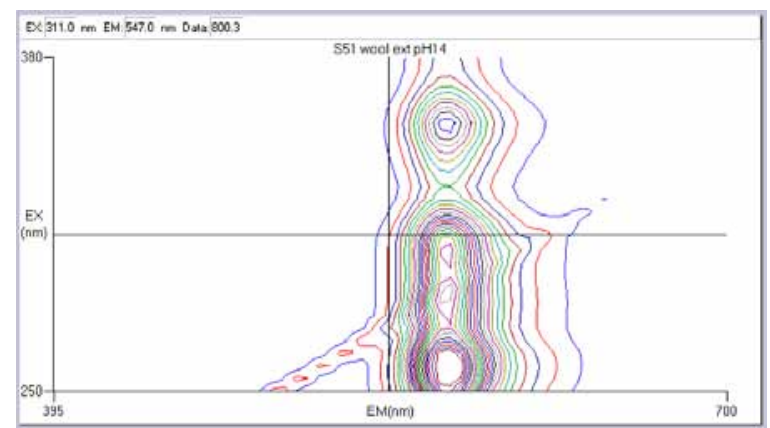

wool
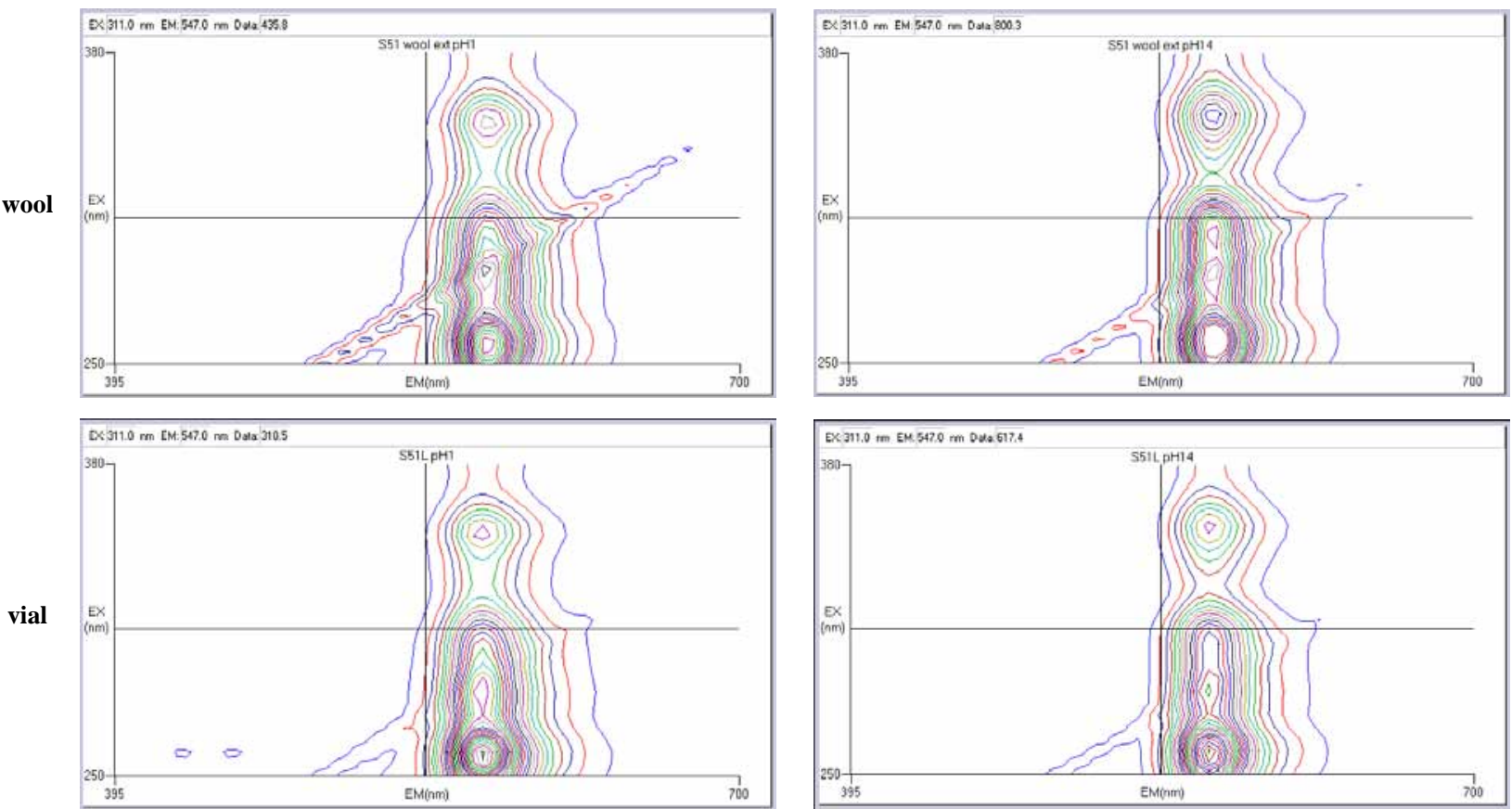

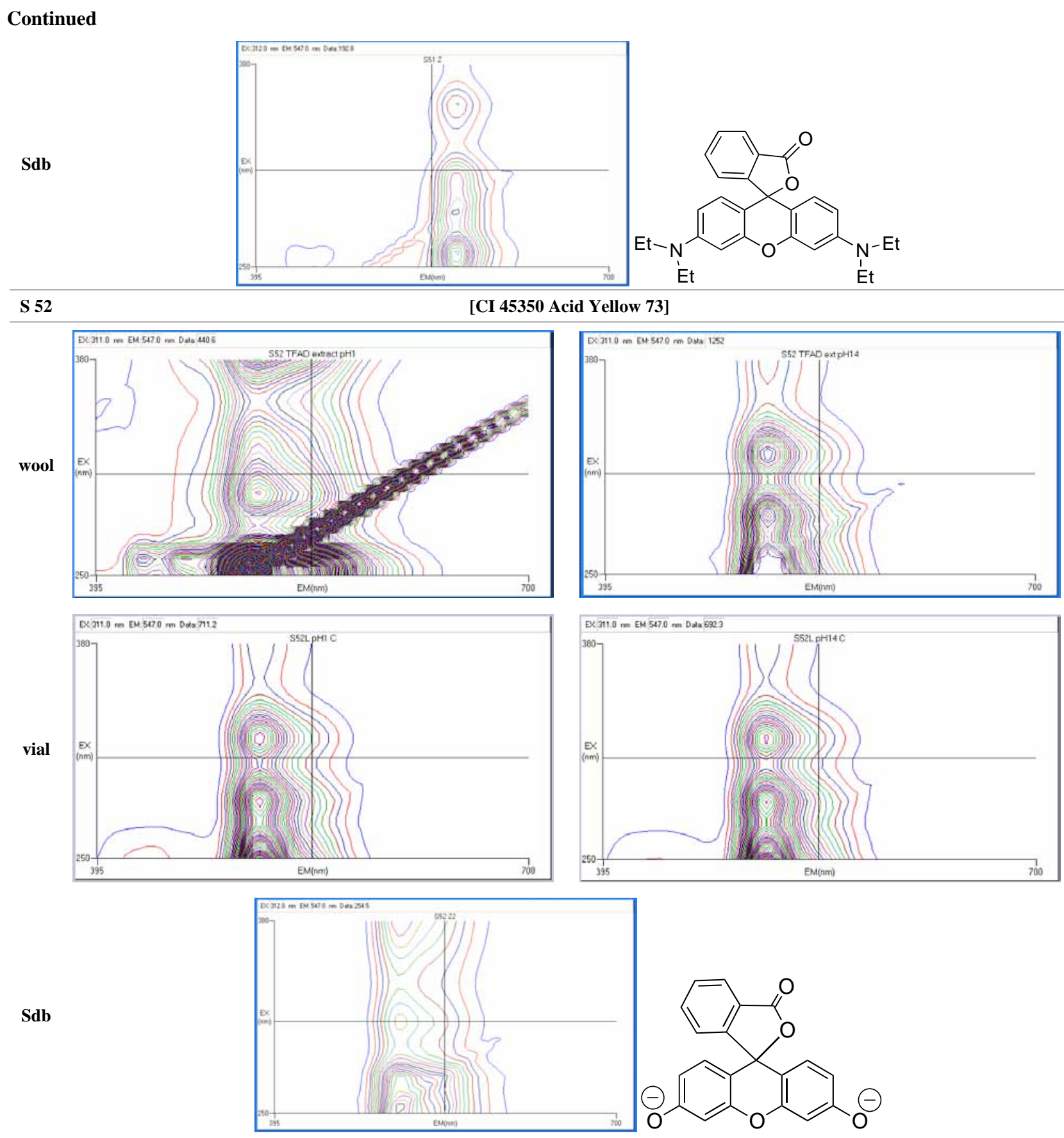

S 53

[CI 45380 Acid Red 87]
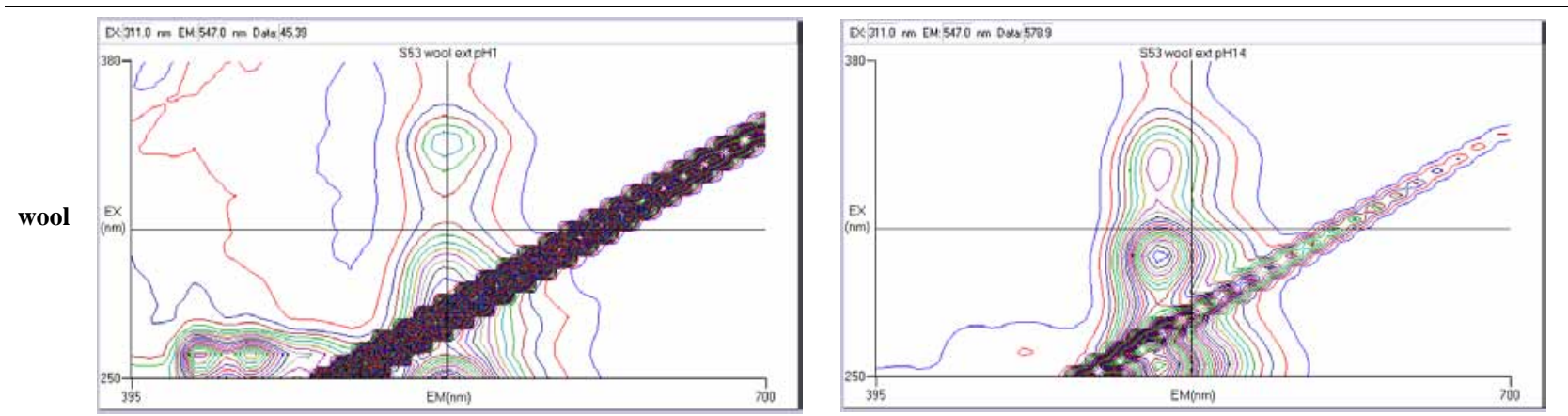


\section{Continued}
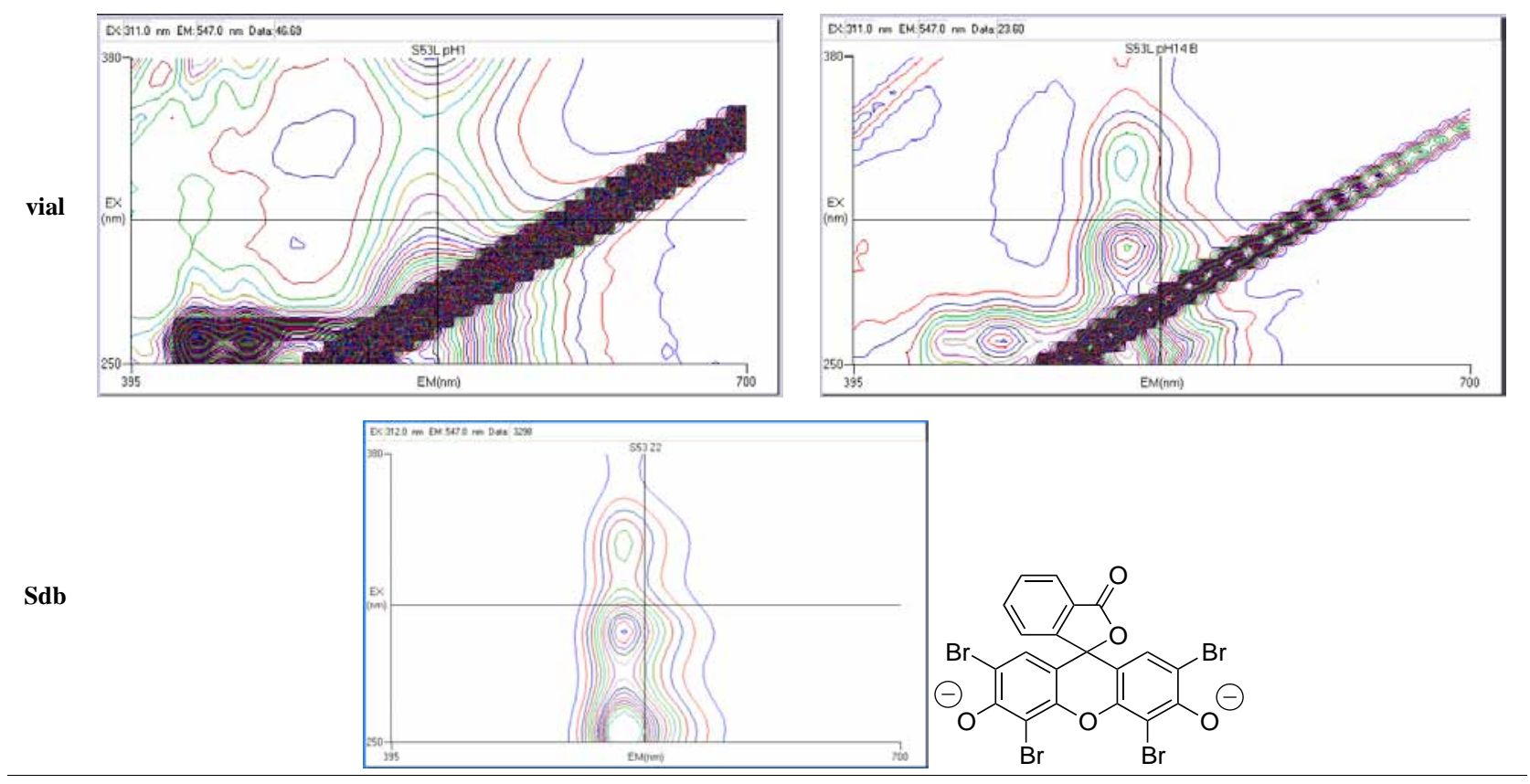

S 55 [CI 47005 Acid Yellow 3]
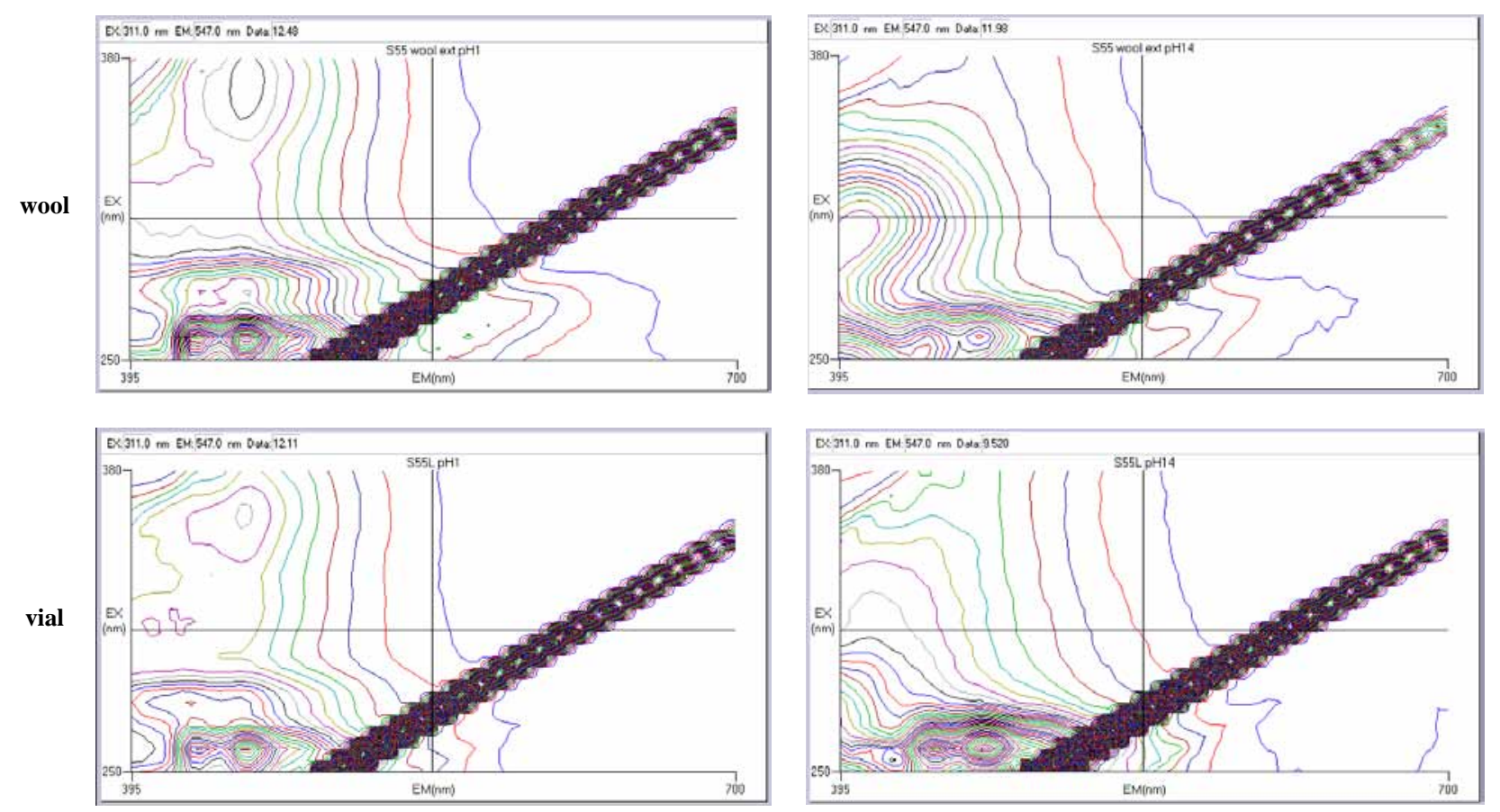

Sdb

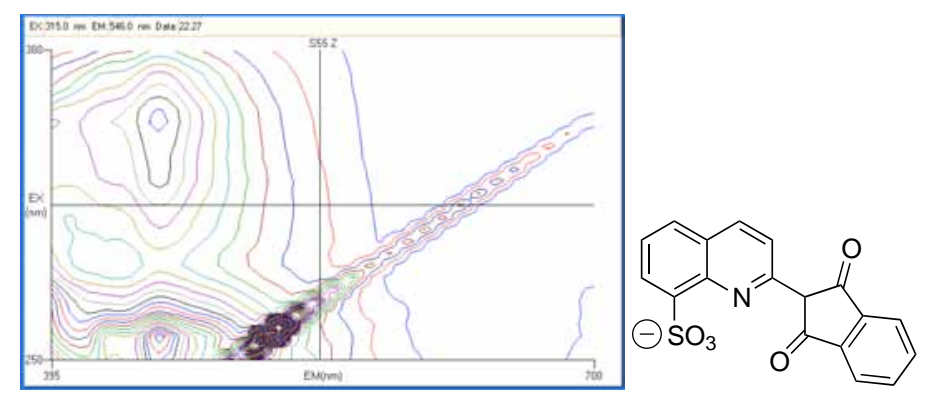




\section{Continued}

S 56

[CI 50240 Basic Red 2]
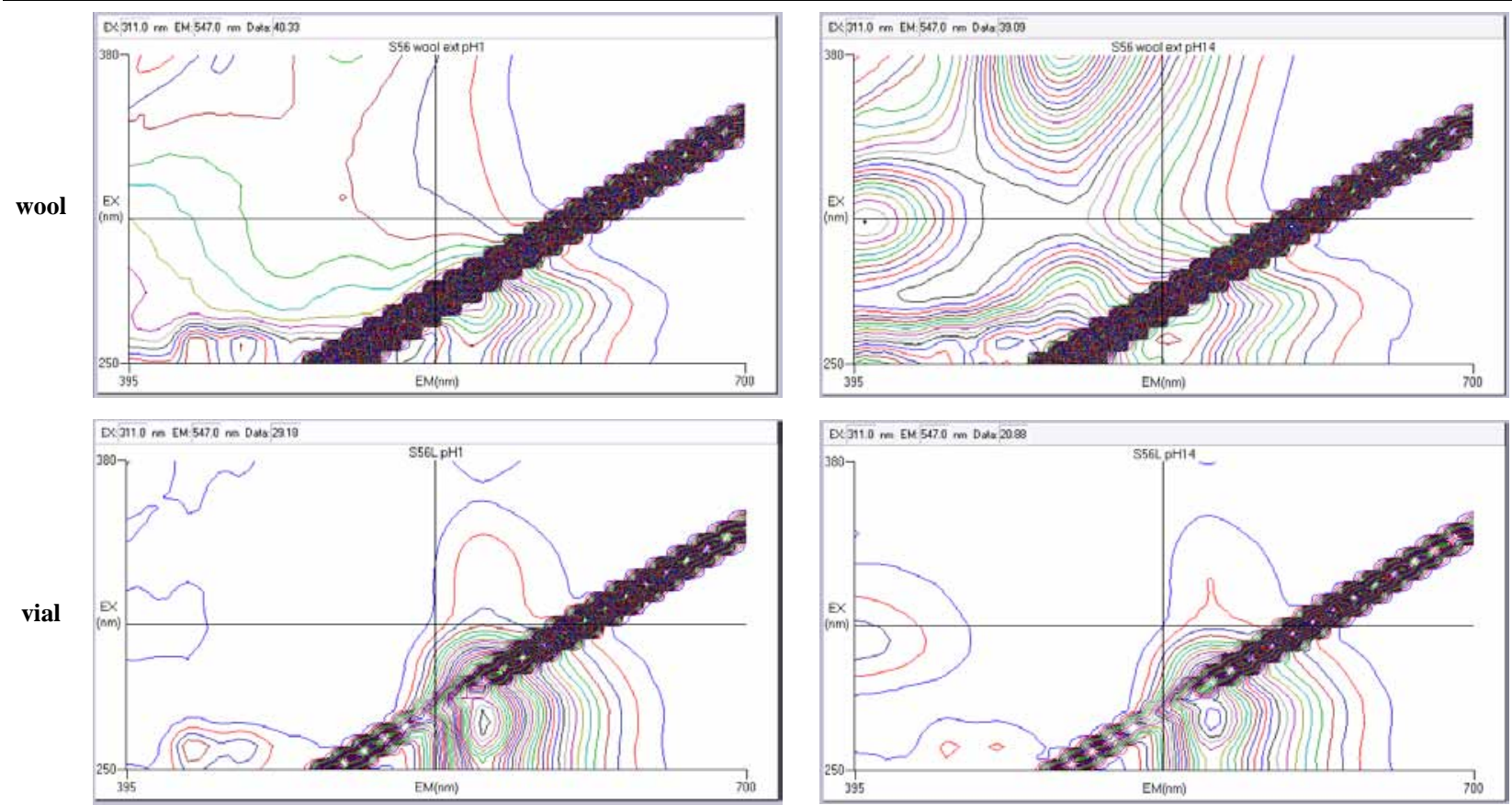

Sdb

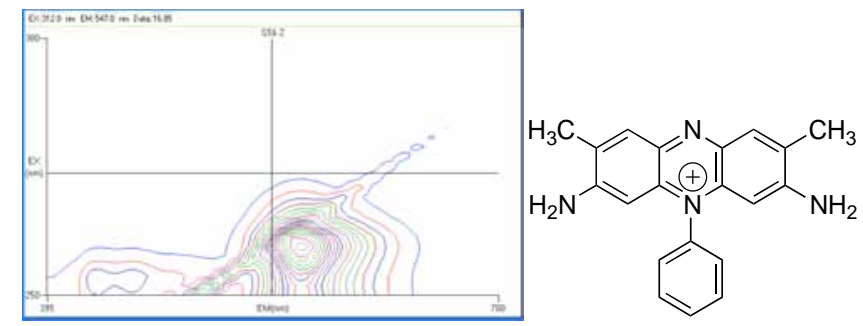

S 58

[CI 50420 Acid Black 2]
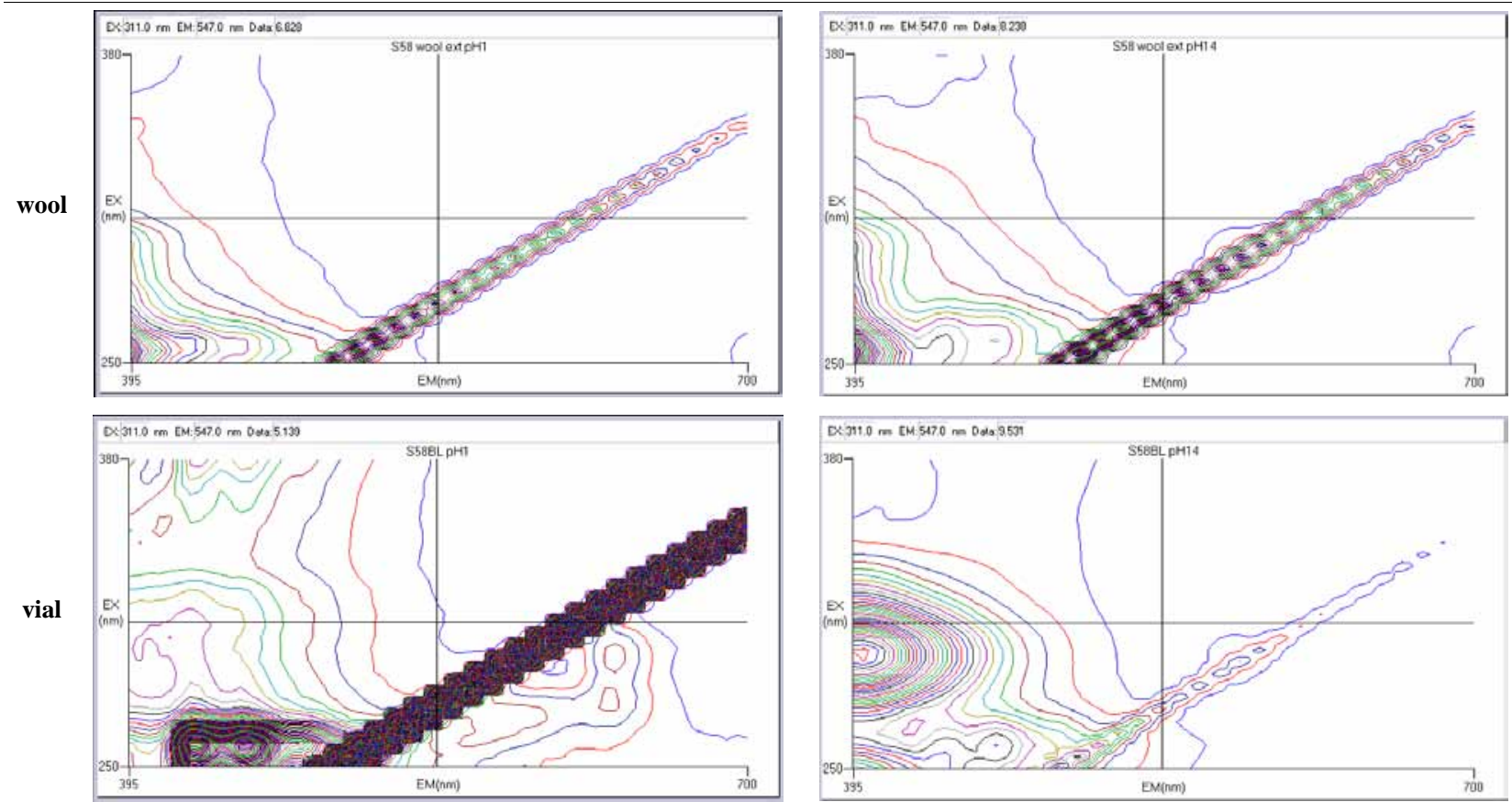

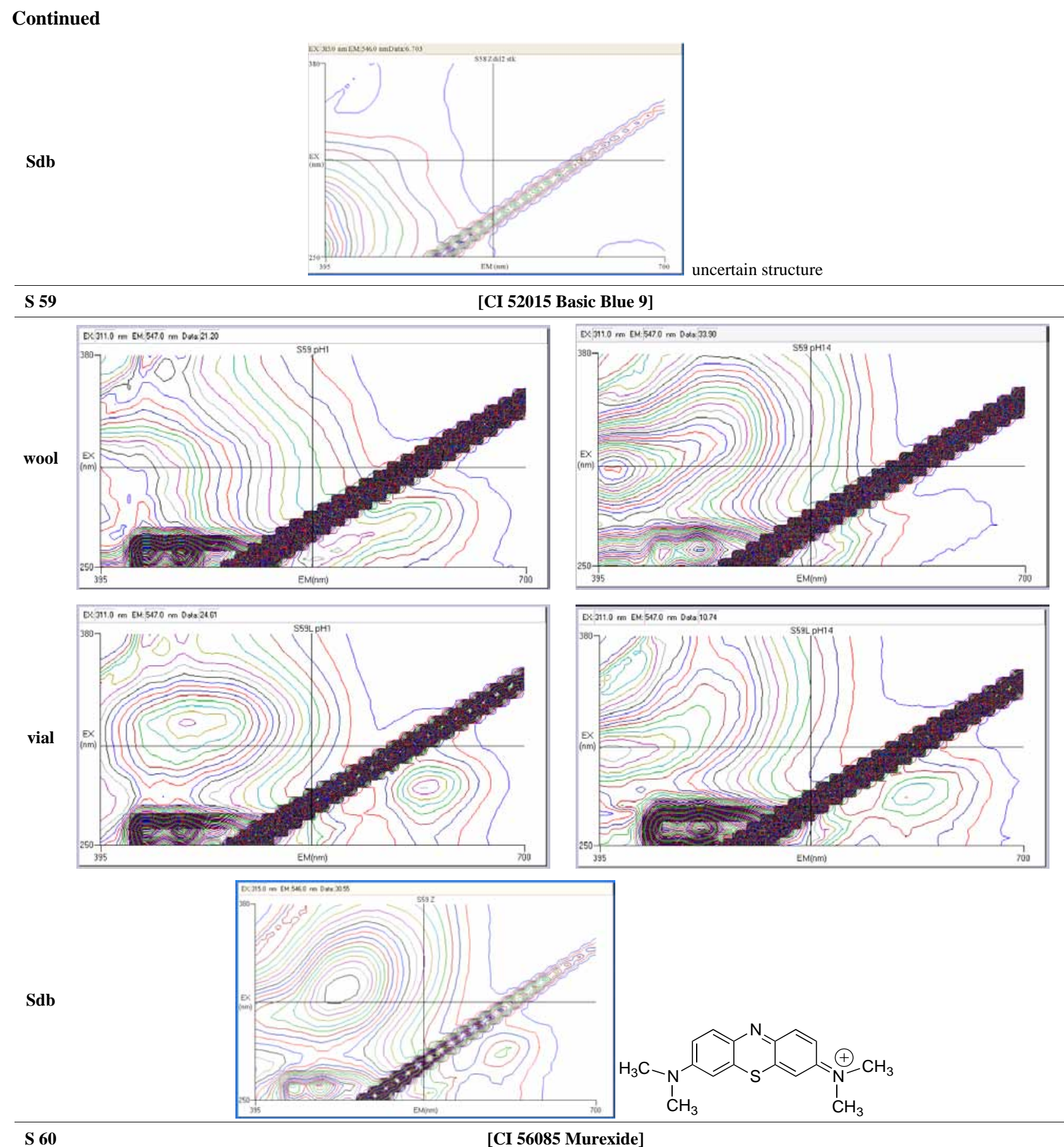

S 60
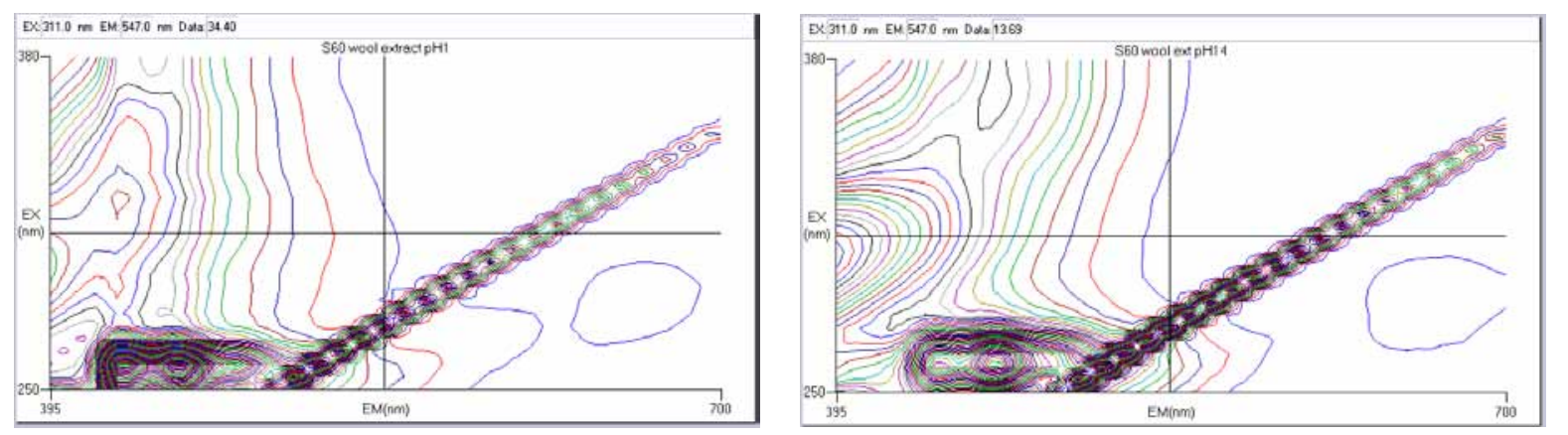


\section{Continued}
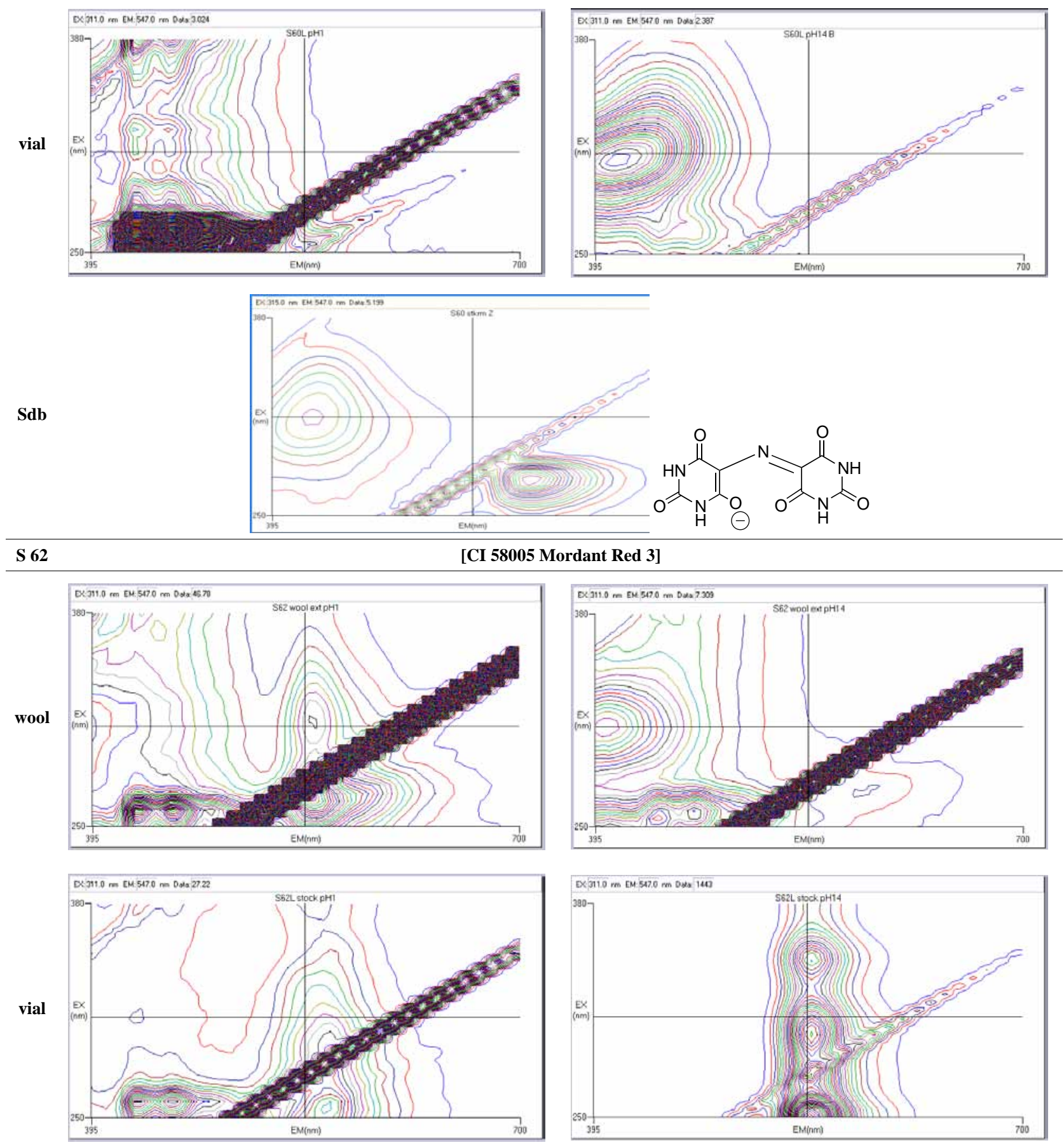

Sdb

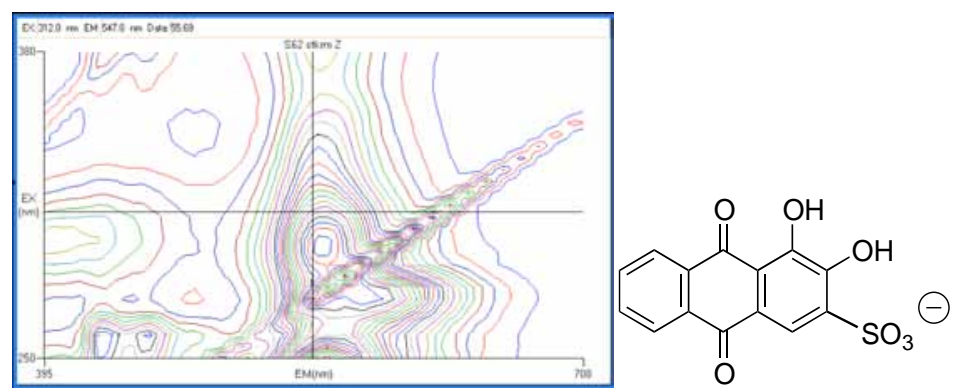




\section{Continued}
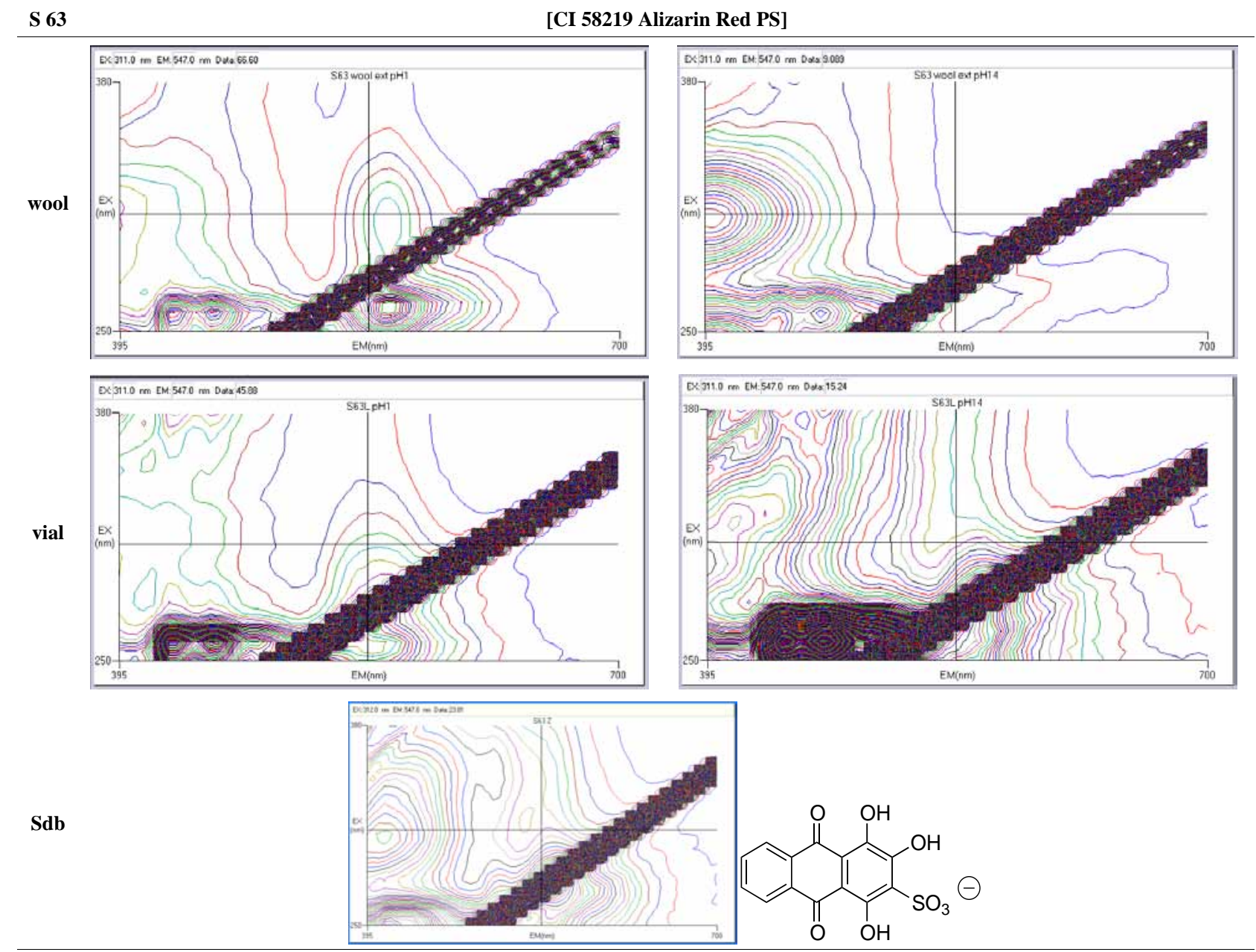

S 64

[CI 58260 Mordant Red 2]
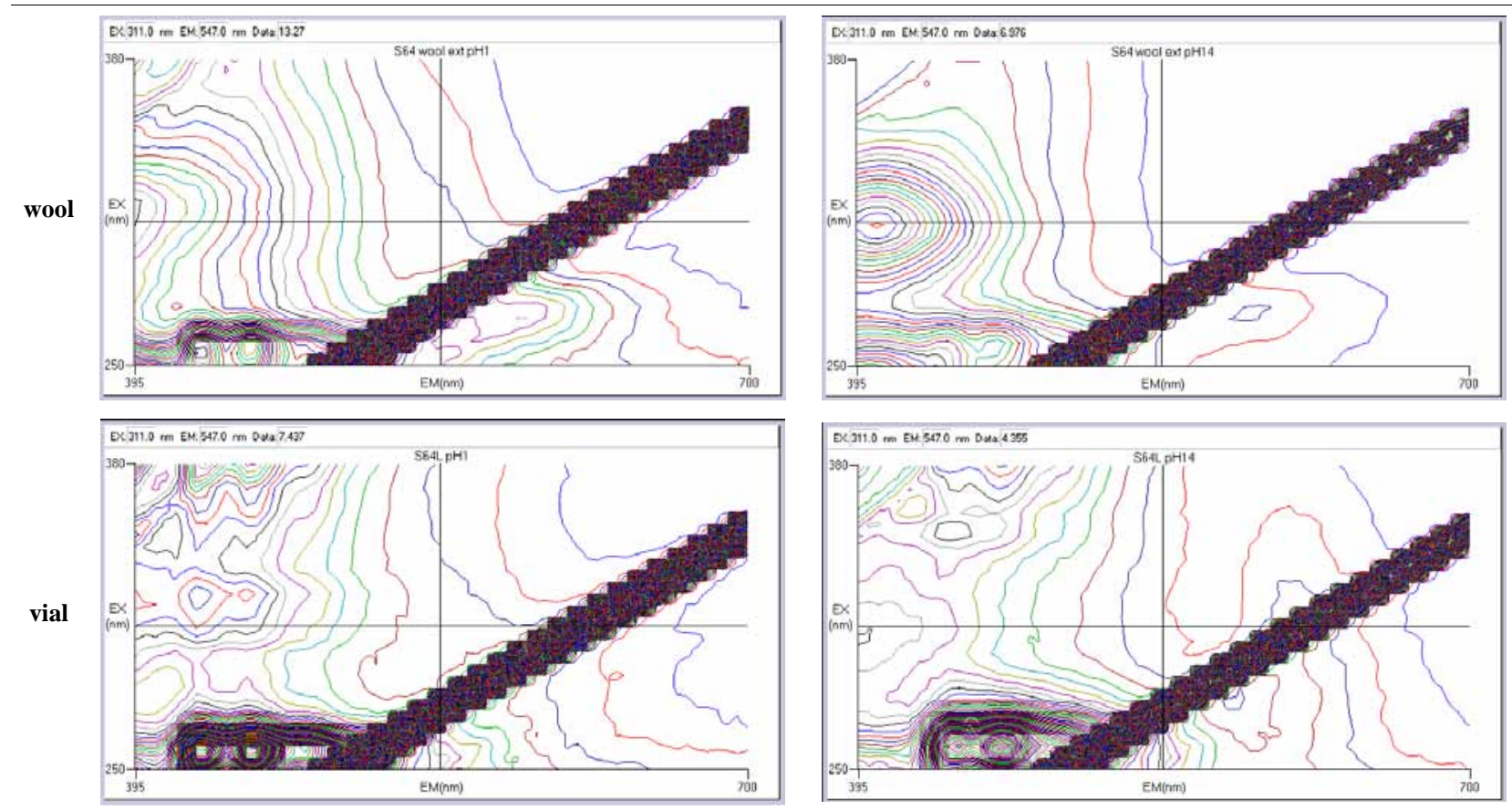

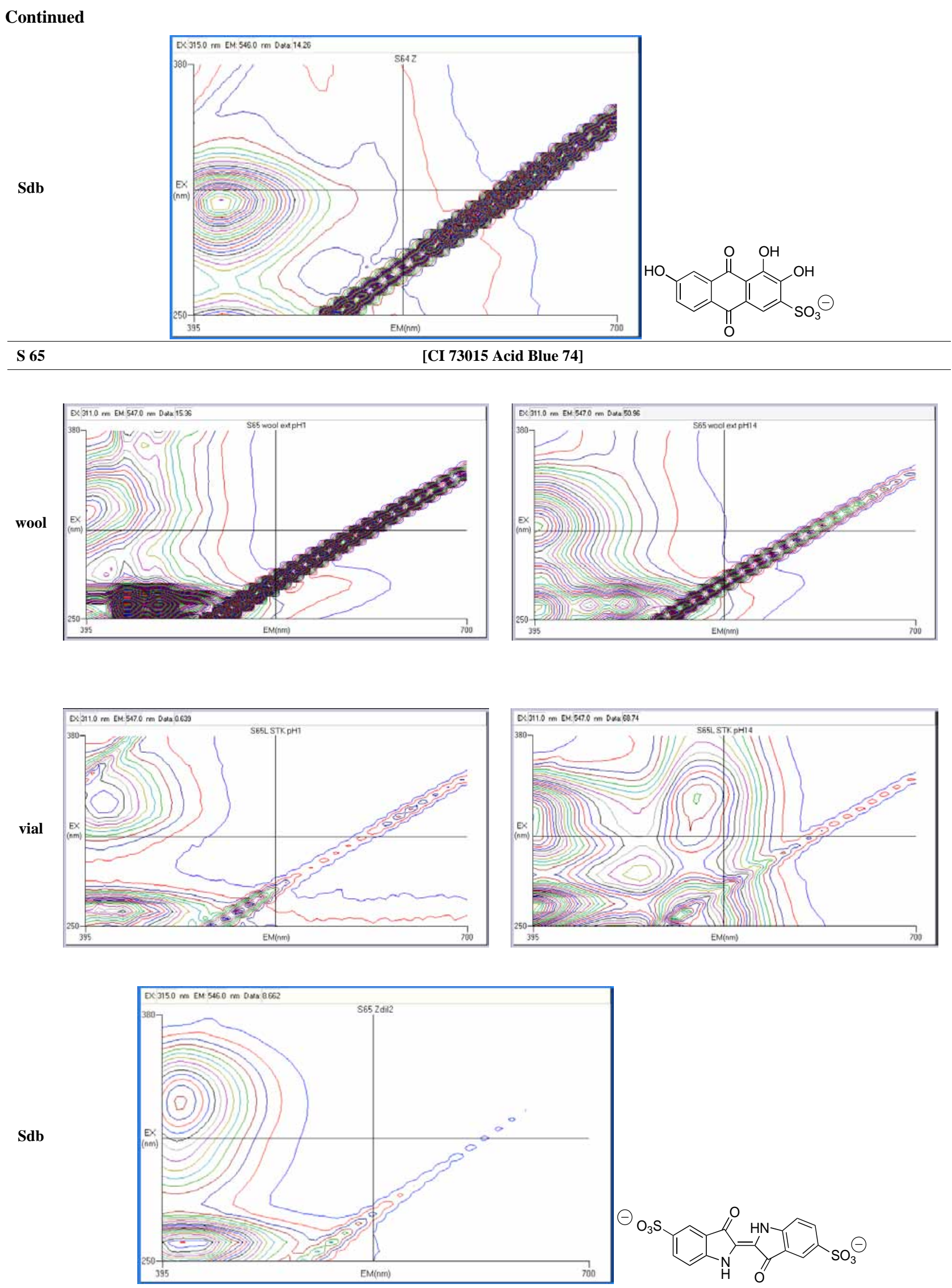




Figure 1. 3D fluorescence spectrum of HPLC grade water showing diagonal features resulting from (left to right) Raman scattering of $1^{\text {st }}$ order Xe lamp photons by water vibration mode $v 3$, Rayleigh scattering of $2^{\text {nd }}$ order Xe lamp photons, and Raman scattering of $2^{\text {nd }}$ order Xe lamp photons by water vibration mode $v 2$. The mask-like artifact in the lower left is a transient feature [6].

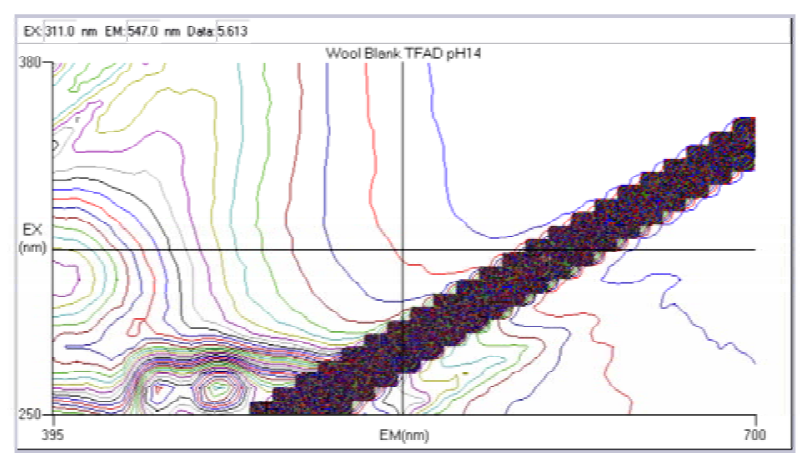

(a)

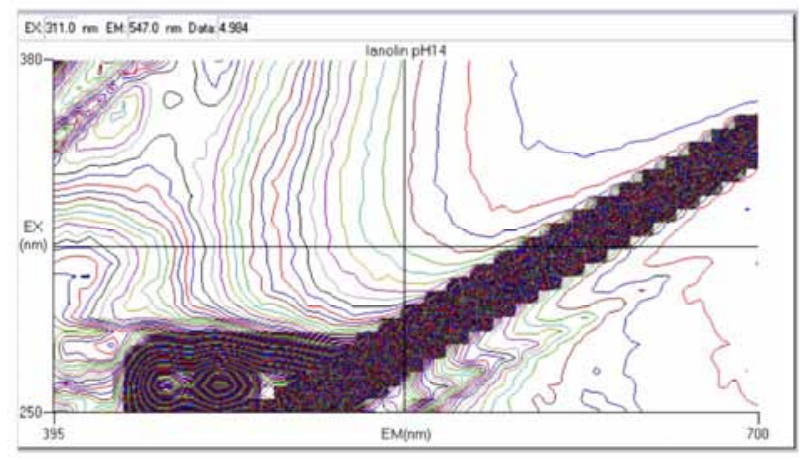

(b)

Figure 2. 3D fluorescence spectrum of (a) TFA extract from undyed wool, adjusted to $\mathrm{pH}$ 14. Peak near $\mathrm{EX}=300 \mathrm{~nm}$ and $\mathrm{EM}=400 \mathrm{~nm}$ has been attributed to lanolin. [7]; (b) TFA/MeOH/lanolin supernatant, adjusted to $\mathrm{pH} 14$.

fluorescence spectra for only 7 of the dyes were unaffected by the change from $\mathrm{pH} 1$ to $\mathrm{pH} 14$. Twenty-four of the Schweppe dyes are actually acid/base indicators [10]. The unbuffered library spectrum in such cases is often recognizably a combination of the $\mathrm{pH} 1$ and $\mathrm{pH} 14$ spectra. To eliminate the ambiguity of dealing with such mixed spectra, we collected fluorescence spectra at the extremes of $\mathrm{pH} 1$ and $\mathrm{pH} 14$ to ensure that the dye molecules were entirely in one molecular form $(\mathrm{pH} 1)$ or the other ( $\mathrm{pH} 14)$.

\subsection{Categories of Spectral Library Matching}

S 51 (Solvent Red 49, Figure 3) typifies a dye for which both the $\mathrm{pH} 1$ and $\mathrm{pH} 14$ extracts match the corresponding library spectra and also are virtually identical with each other. For S 16 (Acid Orange 12, Figure 4), although the acidic and basic spectra are quite different from each other, the wool extract and library spectra nonetheless give excellent matches for both $\mathrm{pH} 1$ and $\mathrm{pH}$ 14.

In contrast, S 41 (Basic Green 1, Figure 5) gives a reasonably good match at $\mathrm{pH} 1$ but not at $\mathrm{pH} 14$; it is possible that the lanolin peak (see Figure 2(a)) is distorting the wool extract spectrum at $\mathrm{pH} 14$. However, there are also dyes, such as S 15 (Acid Red 9, Figure 6), for which the $\mathrm{pH} 14$ spectra match well while the $\mathrm{pH} 1$ spectra do not.

\subsection{Decomposition of Dyes at Extreme pH}

It is known that S 60 (Murexide) irreversibly decomposes at $\mathrm{pH}$ values lower than 4.5 and higher than 9 [11]. However, the rate constant for the decomposition is such that a useable fluorescence spectrum can be obtained if the extraction and spectrum are completed within about 20 min. We did not encounter $\mathrm{pH}$-related decomposition problems with other Schweppe dyes.

\subsection{Guidelines for Dye Identification from 3D Fluorescence Spectra}

To employ the methods described here for identifying an unknown dye, one should extract the dye, adjust the $\mathrm{pH}$, and run the spectra as described in Section 2. Then:

1) Compare the $\mathrm{pH} 14$ and $\mathrm{pH} 1$ spectra with the 46 library ("vial”) spectra in the Supplementary Information-Table 1

[http://simmons.academia.edu/LenSoltzberg]. First look for a match among the $\mathrm{pH} 14$ spectra. If such a match is found, check the corresonding $\mathrm{pH} 1$ spectra for a match as well. These steps may result in a positive identification.

2) If no match is found in step (1), repeat the comparison against the 19 spectra in the Supplementary Information-Table 2

[http://simmons.academia.edu/LenSoltzberg]. Compare the spectra of the unknown sample against only the library spectra, since in Table 2, our extract spectra do not match the library spectra, in several cases probably 


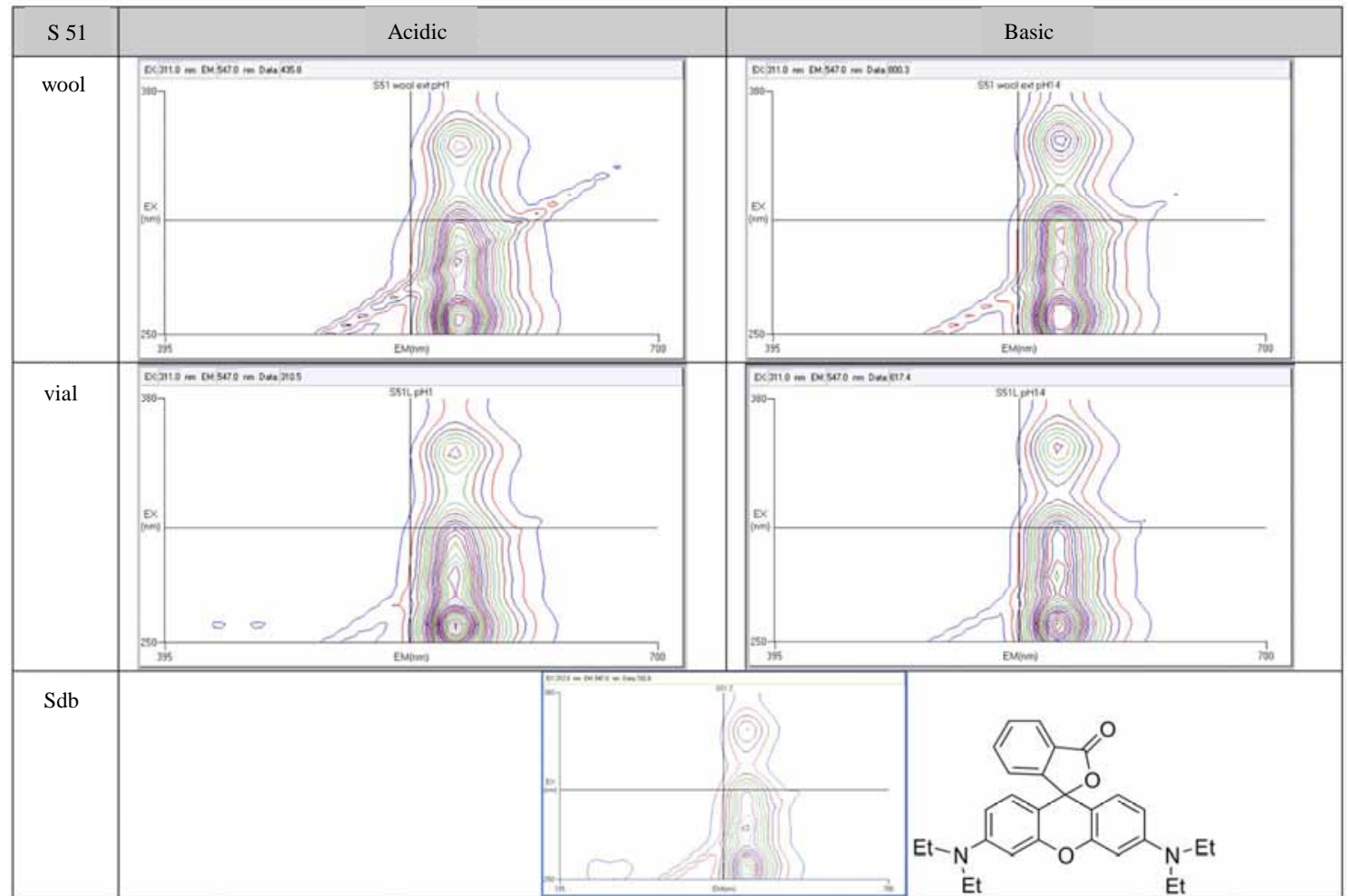

Figure 3. Dye extract showing no pH dependence of the 3D fluorescence spectrum, pH 1 (left) versus pH 14 (right). Excellent match between wool extract (“wool”, upper) and library spectra ("vial”, lower). Unbuffered Schweppe database spectrum (Sdb) is at bottom next to the structure. S 51 is CI 45170 (Solvent Red 49).

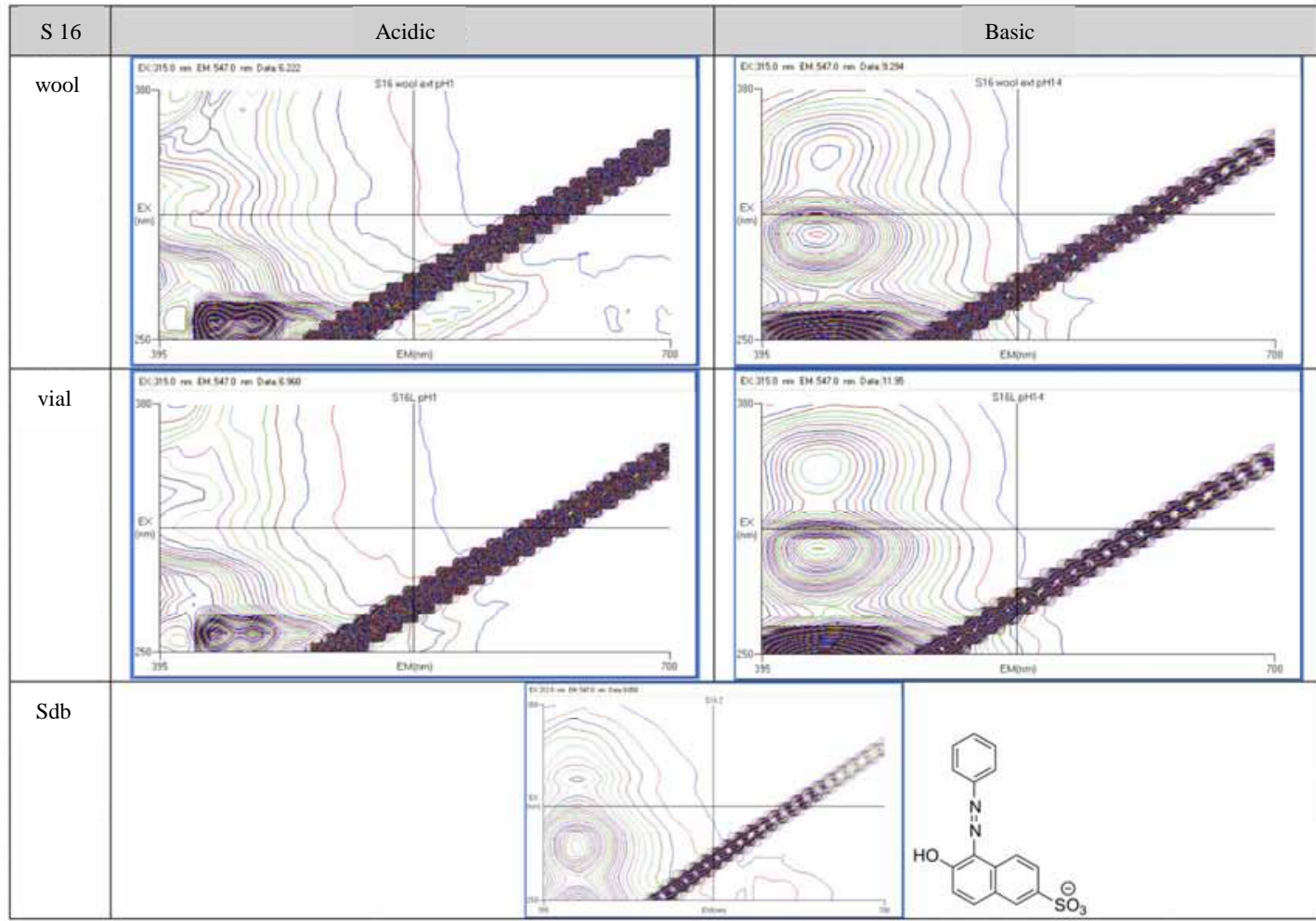

Figure 4. Dye extract showing pH dependence of the 3D fluorescence spectrum. Excellent match between wool extract and library spectra. S 16 is CI 15970 (Acid Orange 12). 


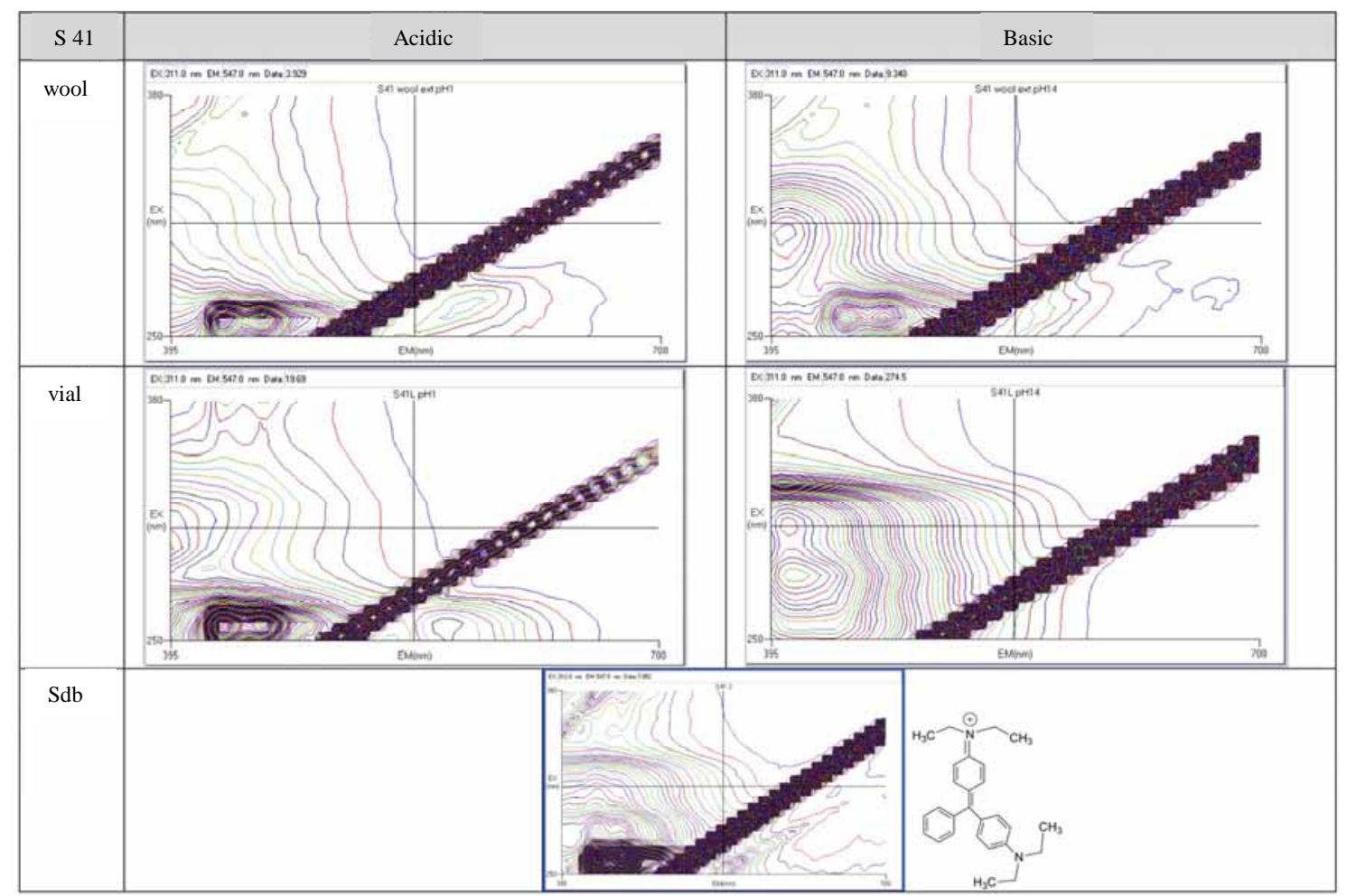

Figure 5. Dye extract showing good match between pH 1 wool extract and pH 1 library spectrum. S 41 is CI 42040 (Basic Green 1).

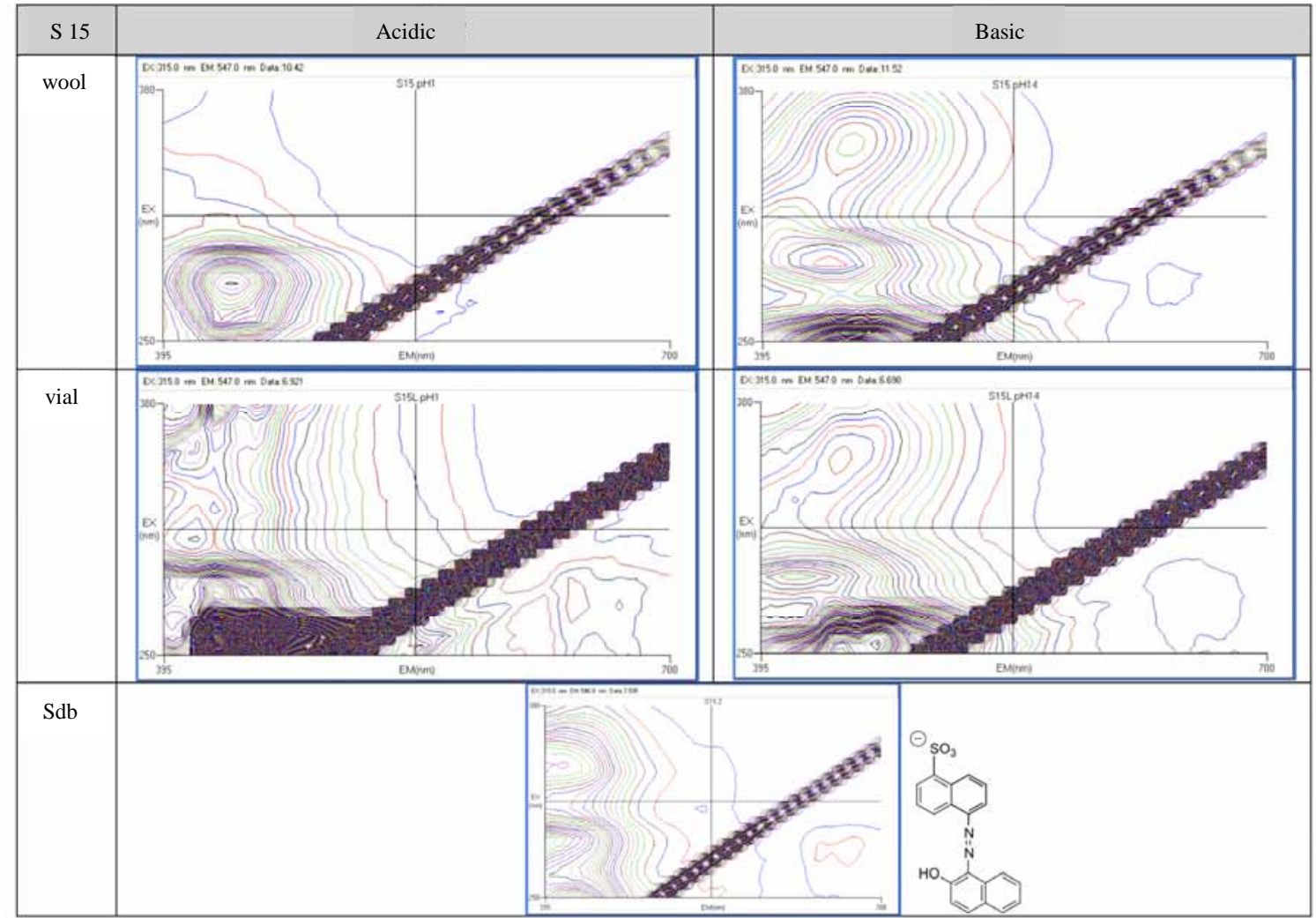

Figure 6. Dye extract showing good match between pH 14 wool extract and pH 14 library spectrum. S 15 is CI 15635 (Acid Red 9). 
Table 2. Dye cannot be identified from 3D fluorescence spectra.

\begin{tabular}{|c|c|c|}
\hline & Acidic & Basic \\
\hline $\mathbf{S} \mathbf{x}$ & \multicolumn{2}{|c|}{ [CI (Colour Index) Number CI Name] } \\
\hline wool & dye extracted from wool fibers $-\mathrm{pH} 1$ & dye extracted from wool fibers $-\mathrm{pH} 14$ \\
\hline vial & dye from solution library $-\mathrm{pH} 1$ & dye from solution library $-\mathrm{pH} 14$ \\
\hline Sdb & dye from solution library - no $\mathrm{pH}$ adjustment & dye structure \\
\hline S 2 & & id Yellow 24] \\
\hline
\end{tabular}
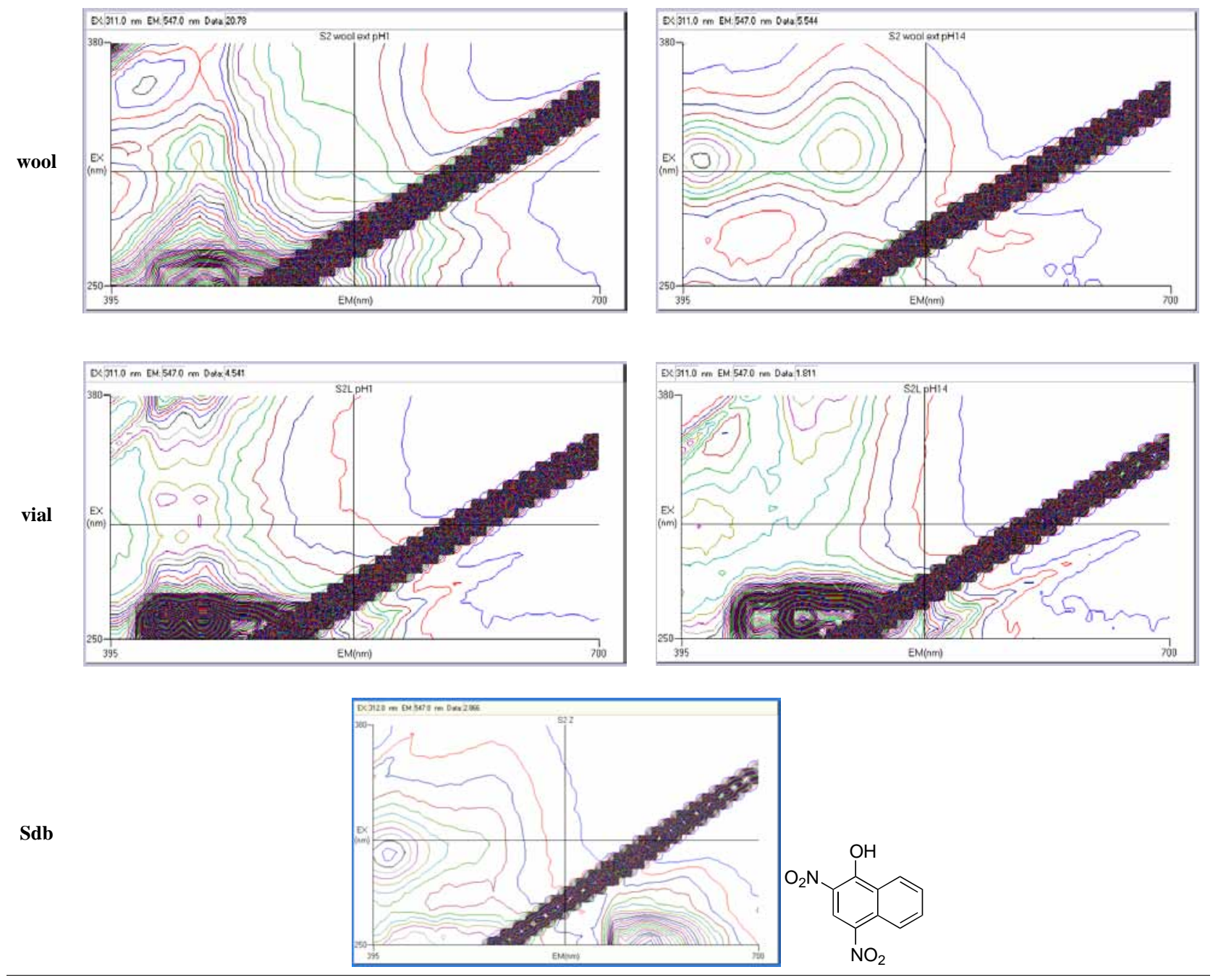

S 5

[CI 13065 Acid Yellow 36]
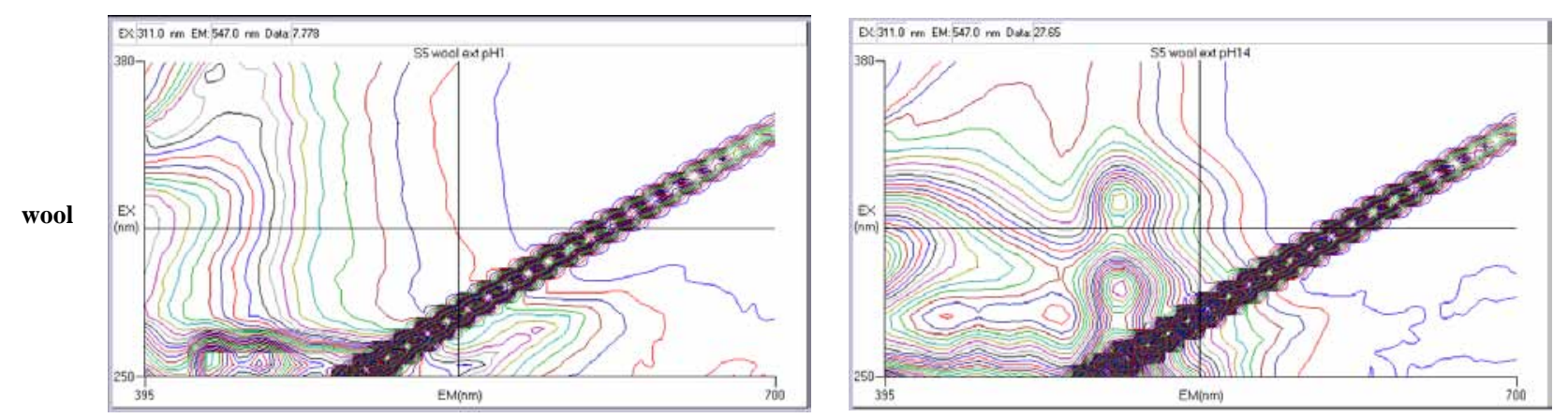


\section{Continued}
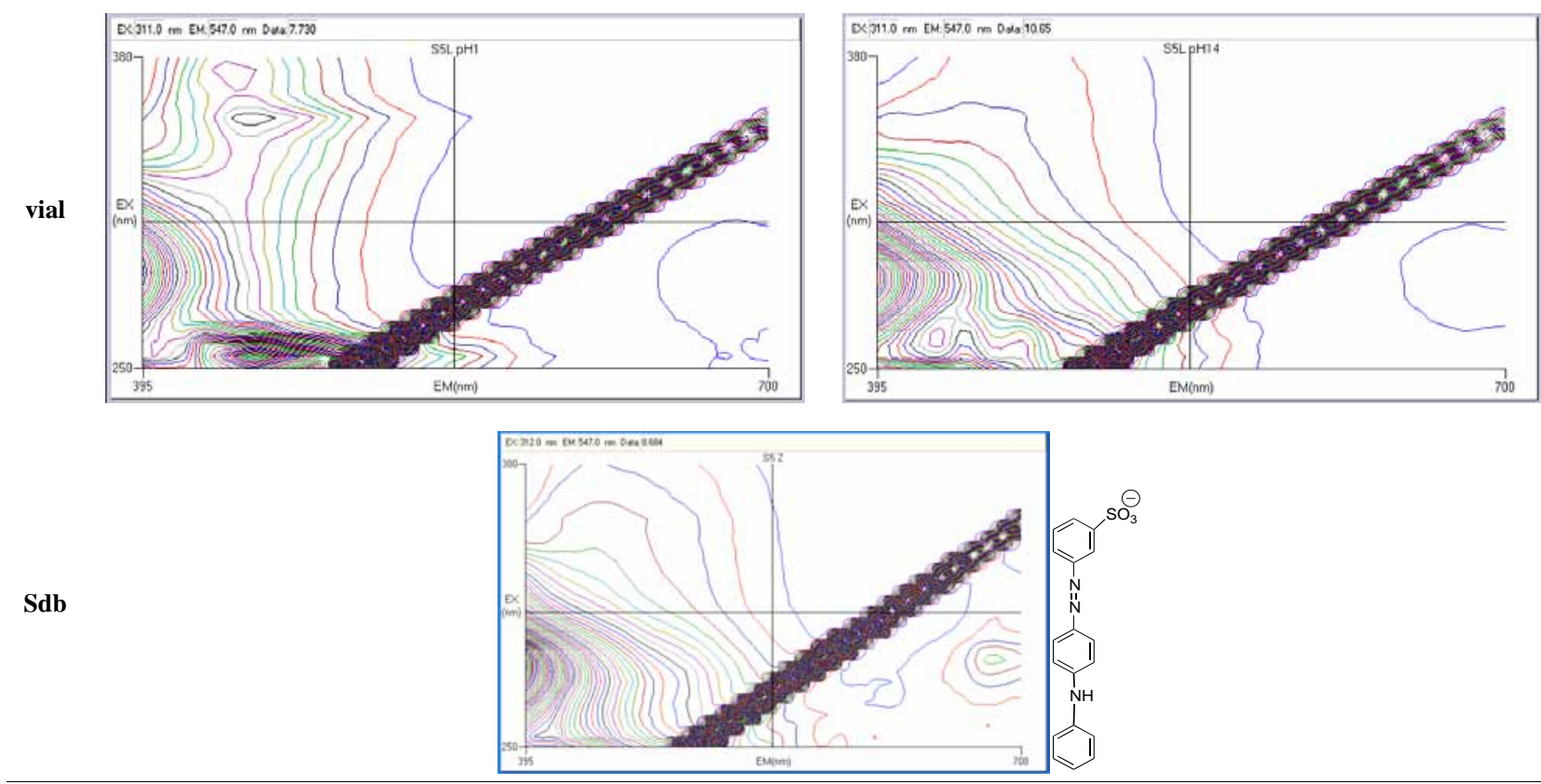

S 8

[CI 13355 Acid Red 74]
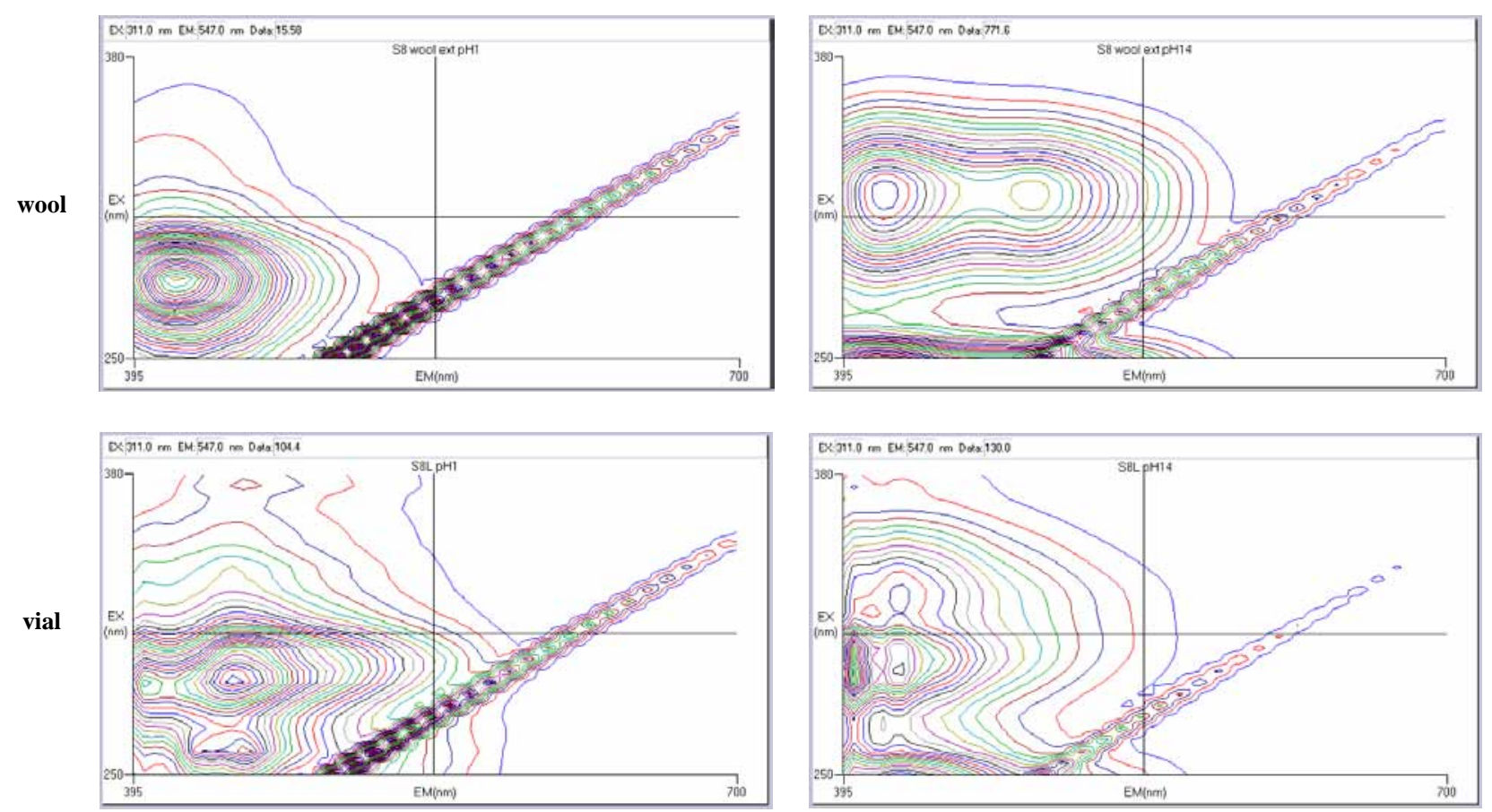

Sdb






\section{Continued}
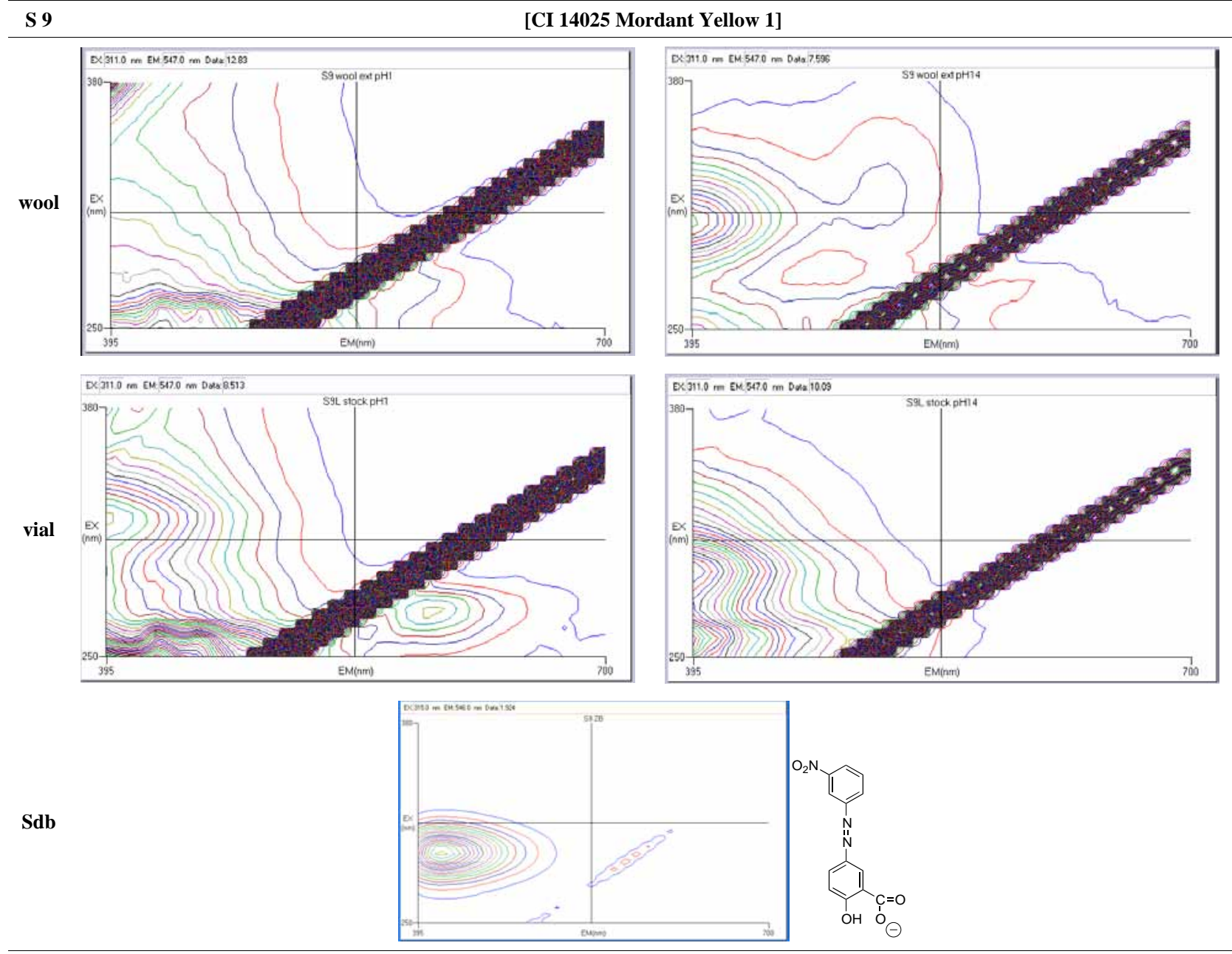

S 10

[CI 14270 Acid Orange 6]
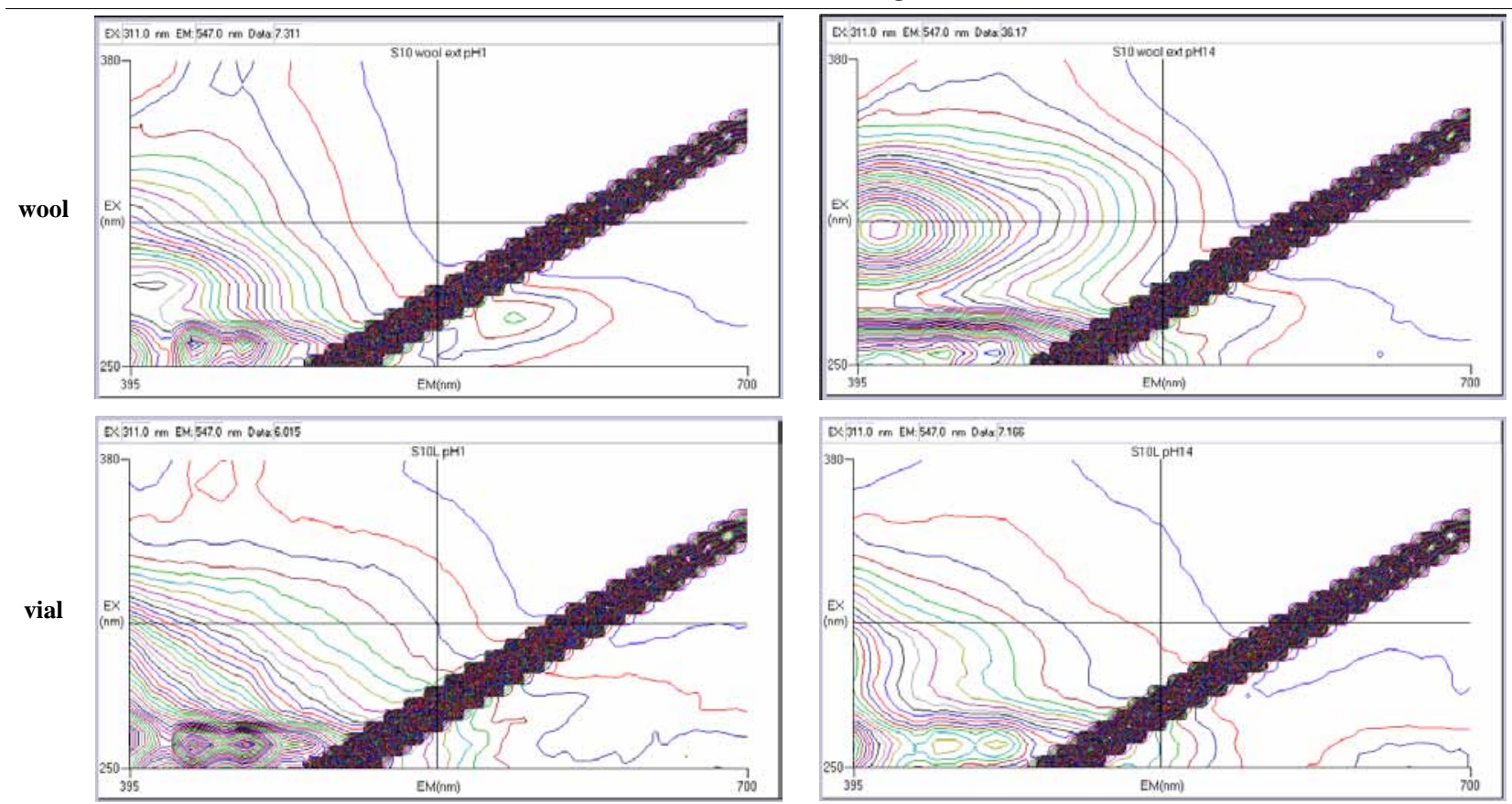

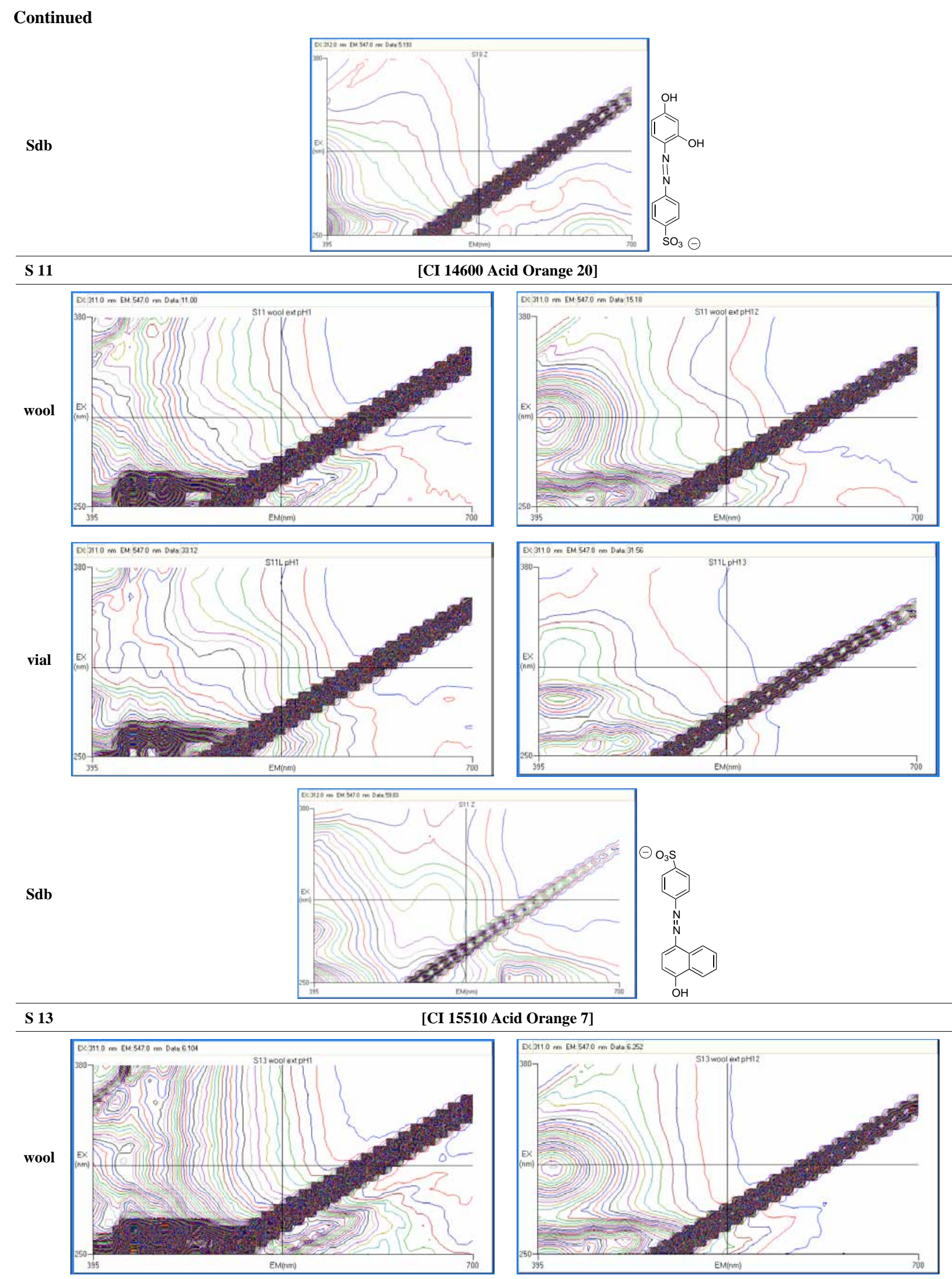


\section{Continued}
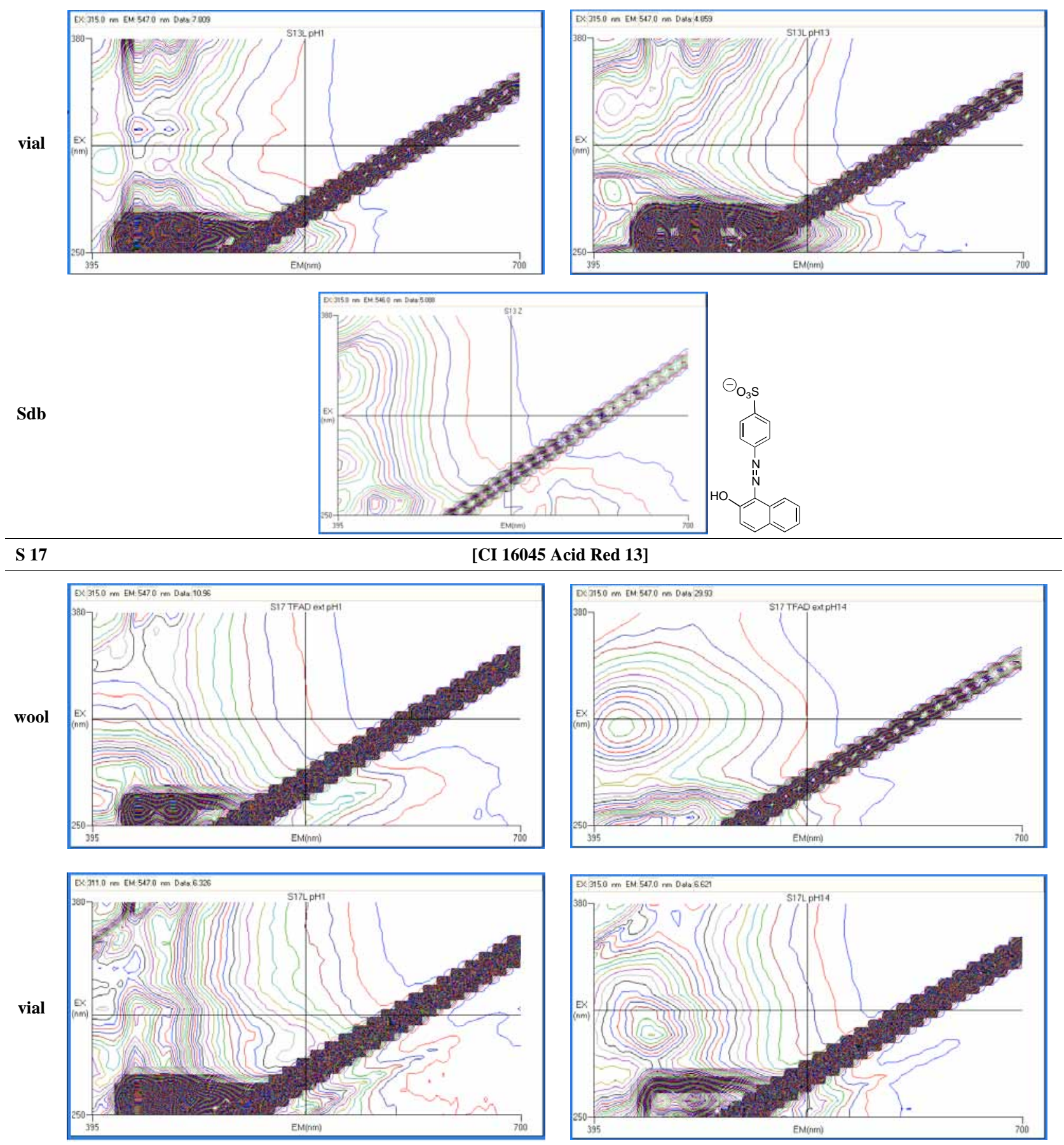

Sdb

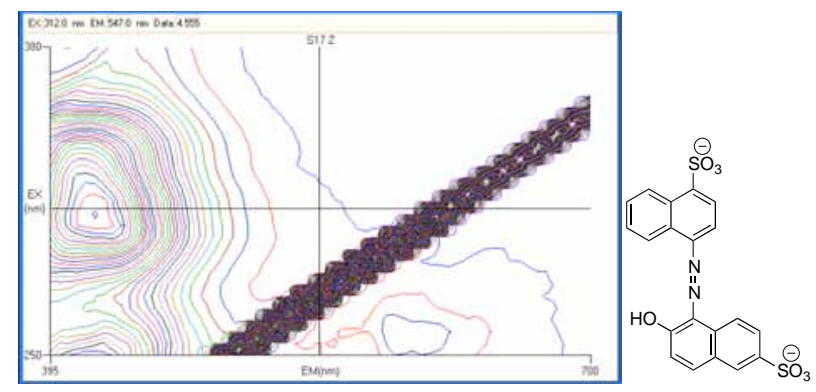




\section{Continued}

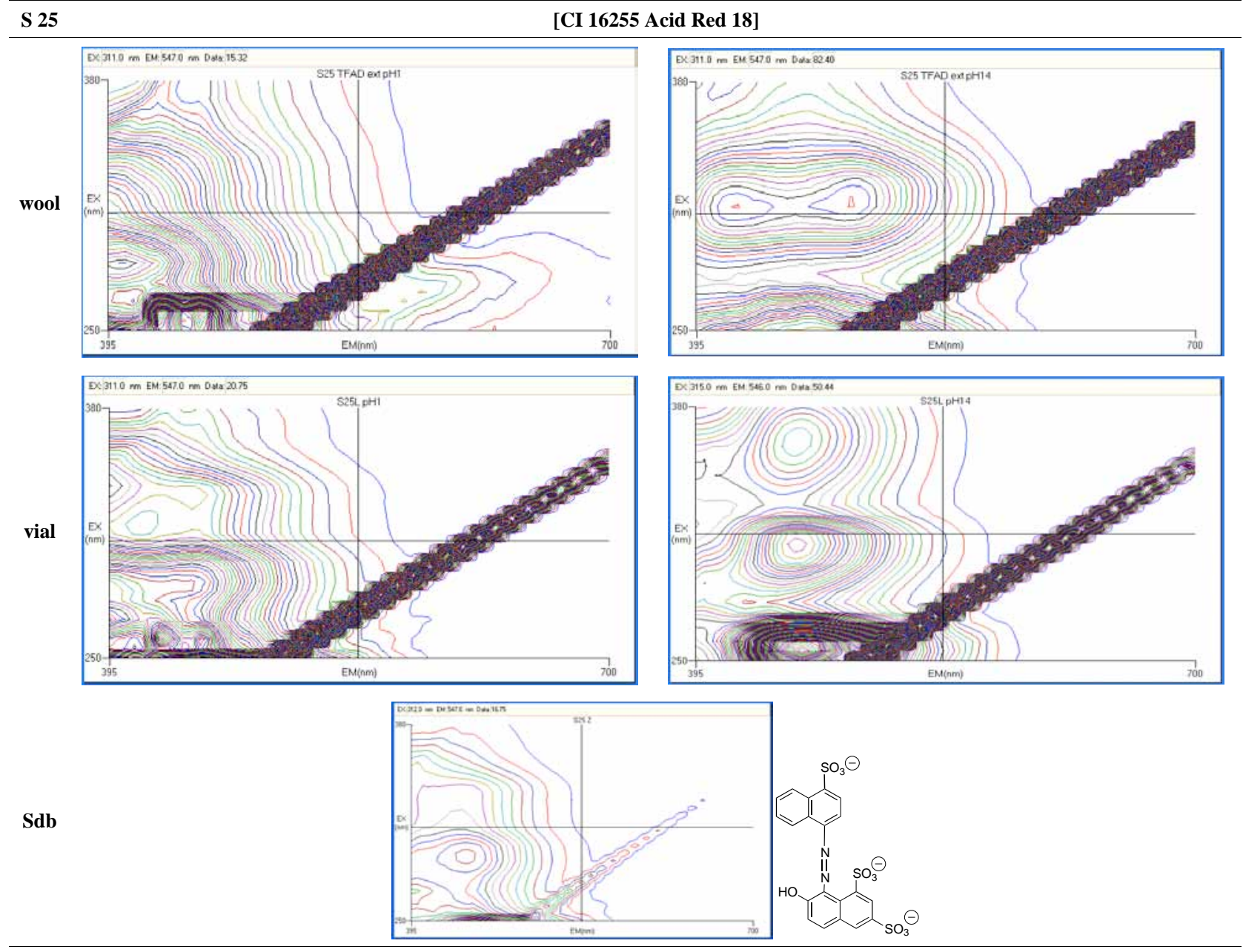

S 29

[CI 18055 Acid Violet 7]
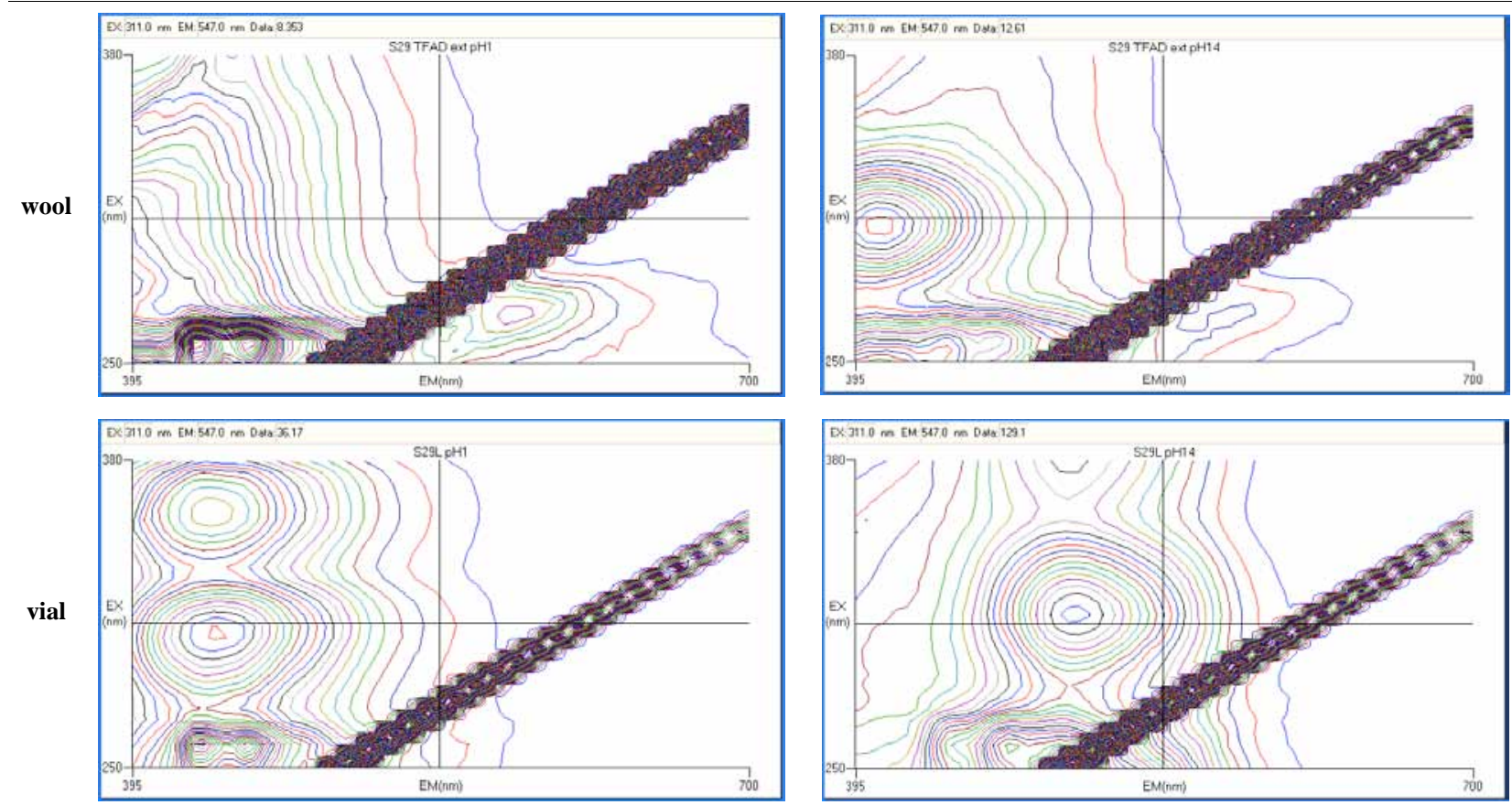


\section{Continued}

Sdb
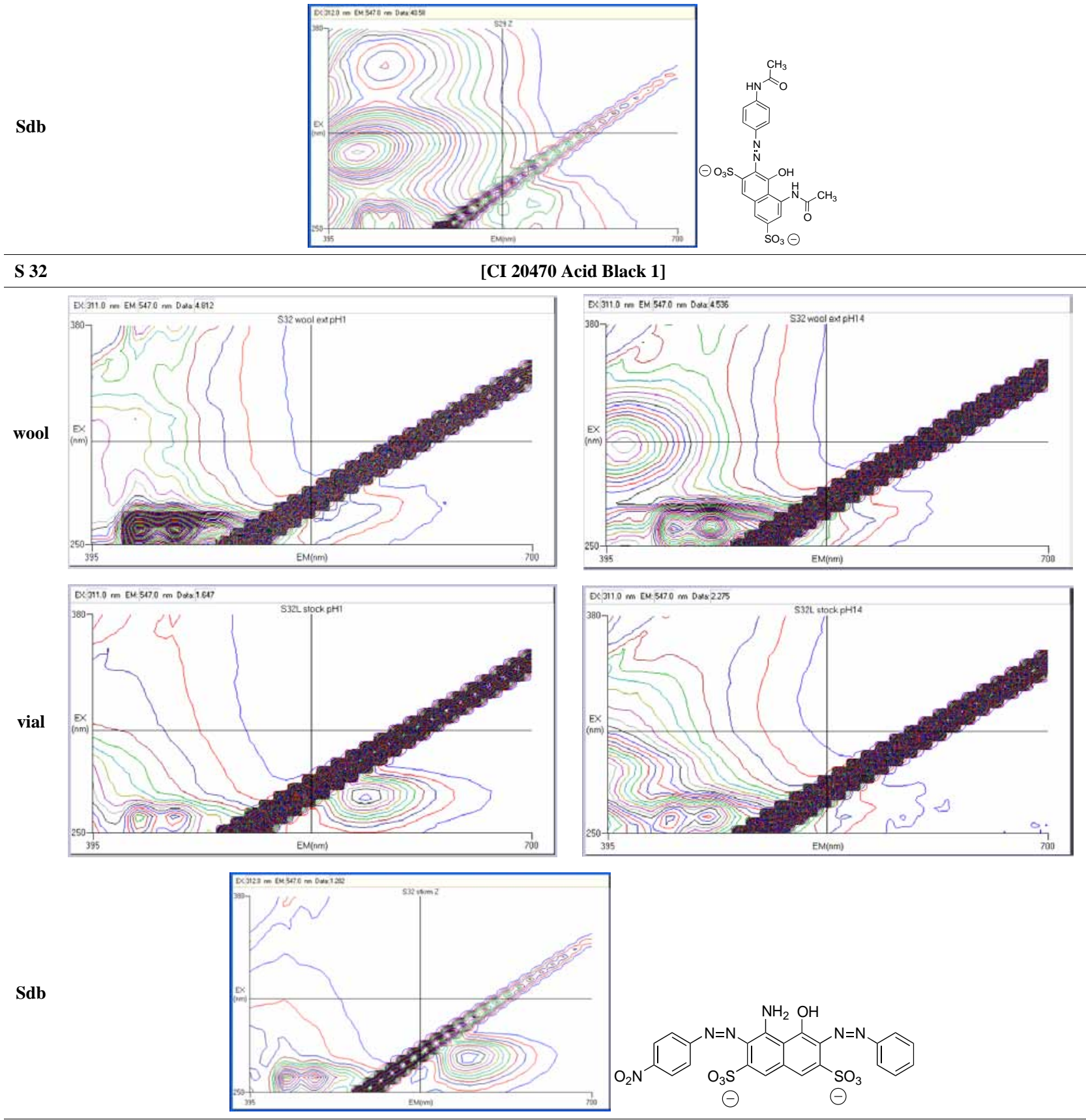

S 37

[CI 27200 Acid Red 115]
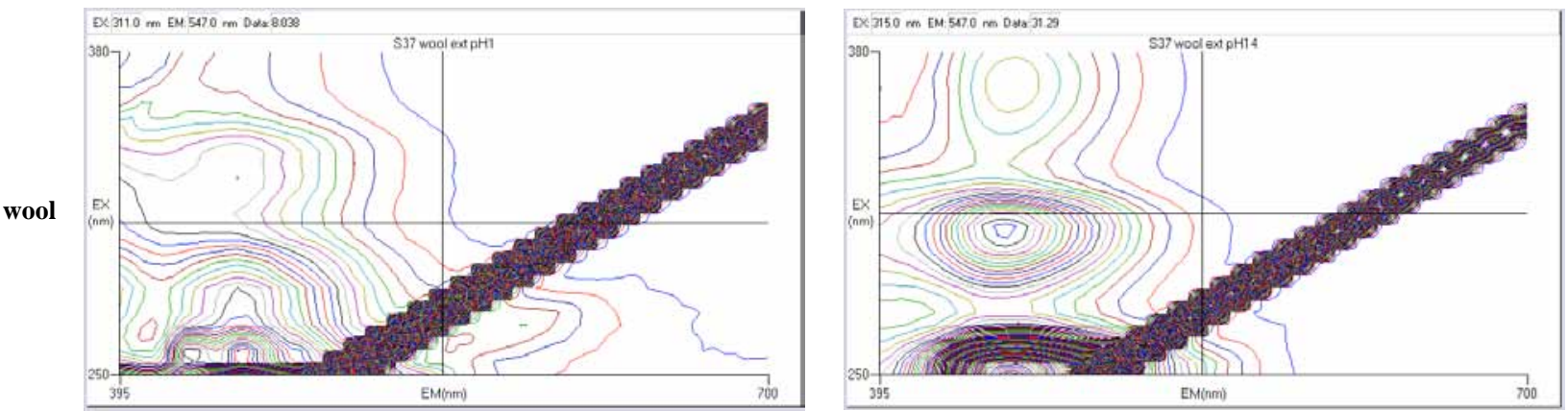


\section{Continued}
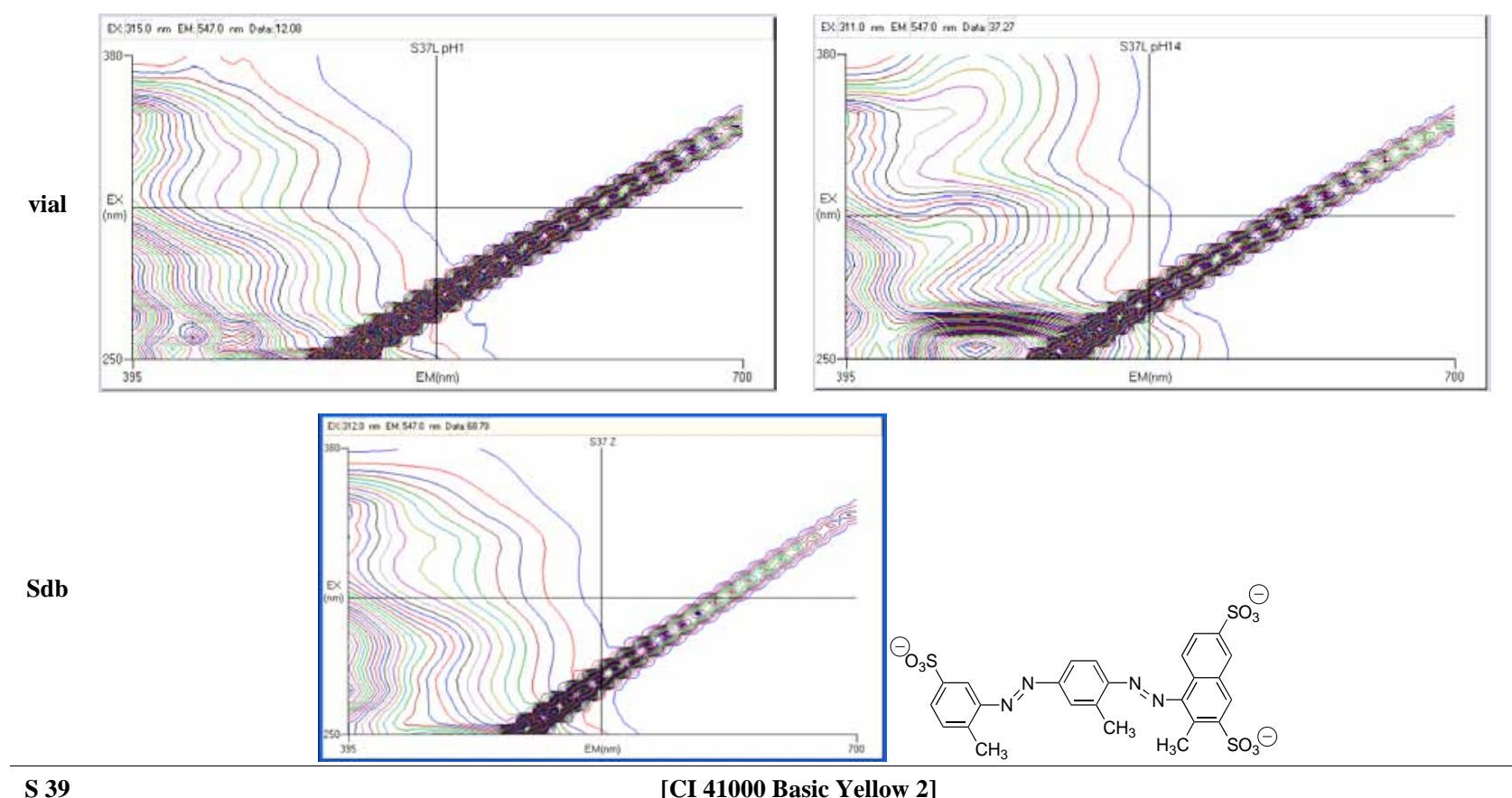

S 39

[CI 41000 Basic Yellow 2]
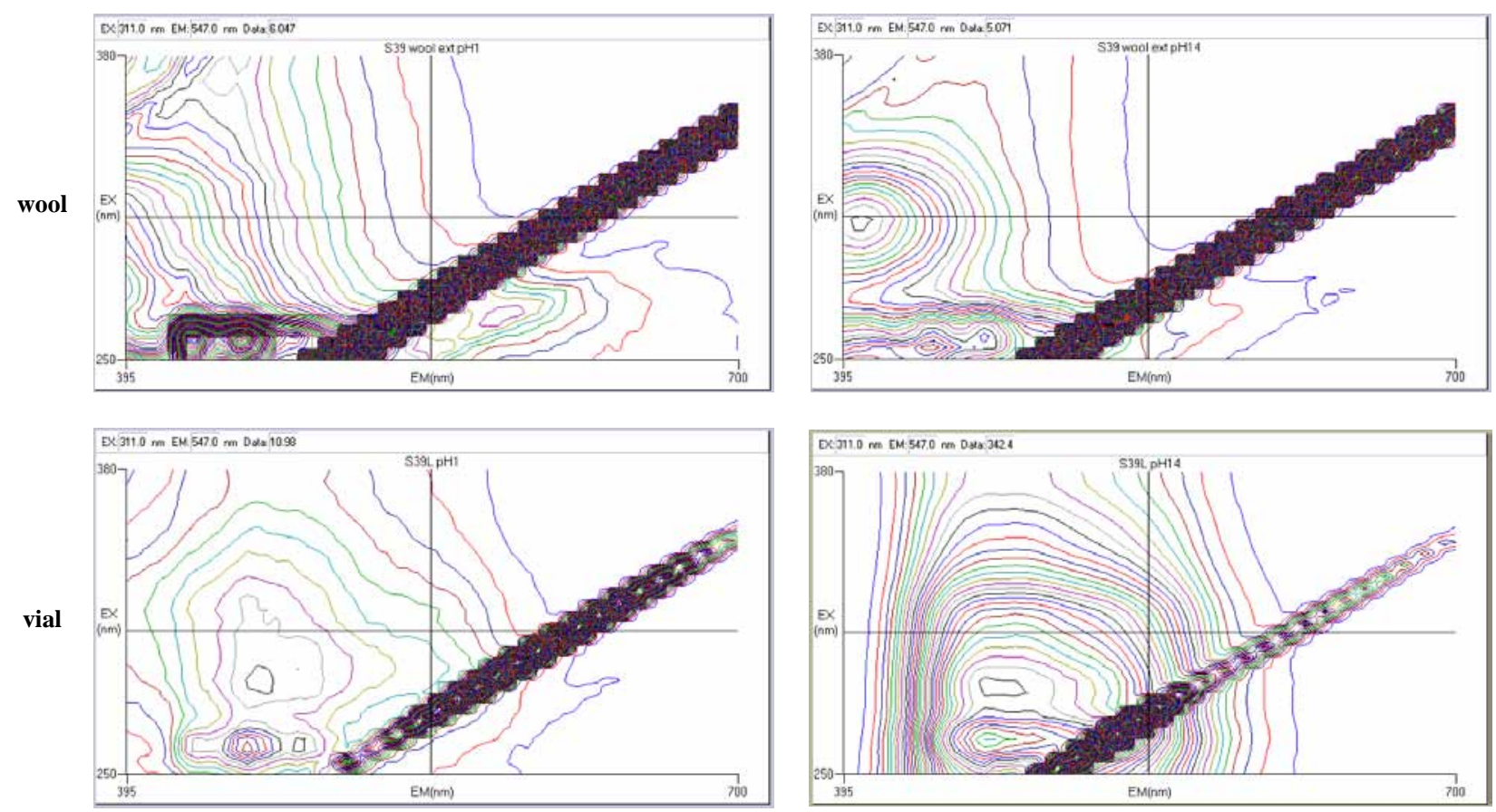

Sdb

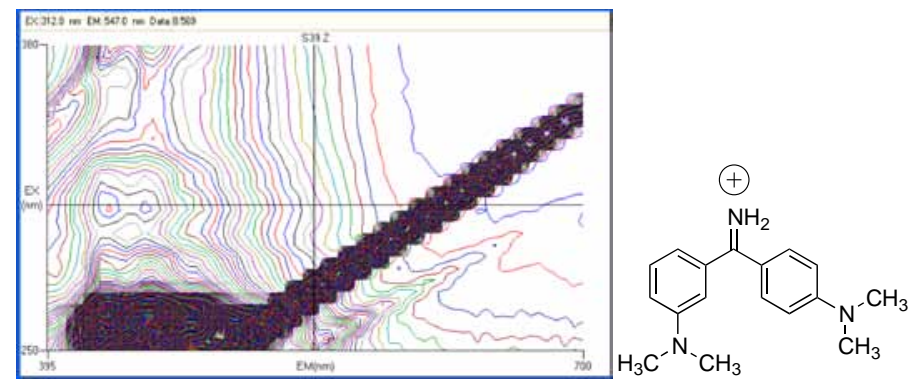




\section{Continued}
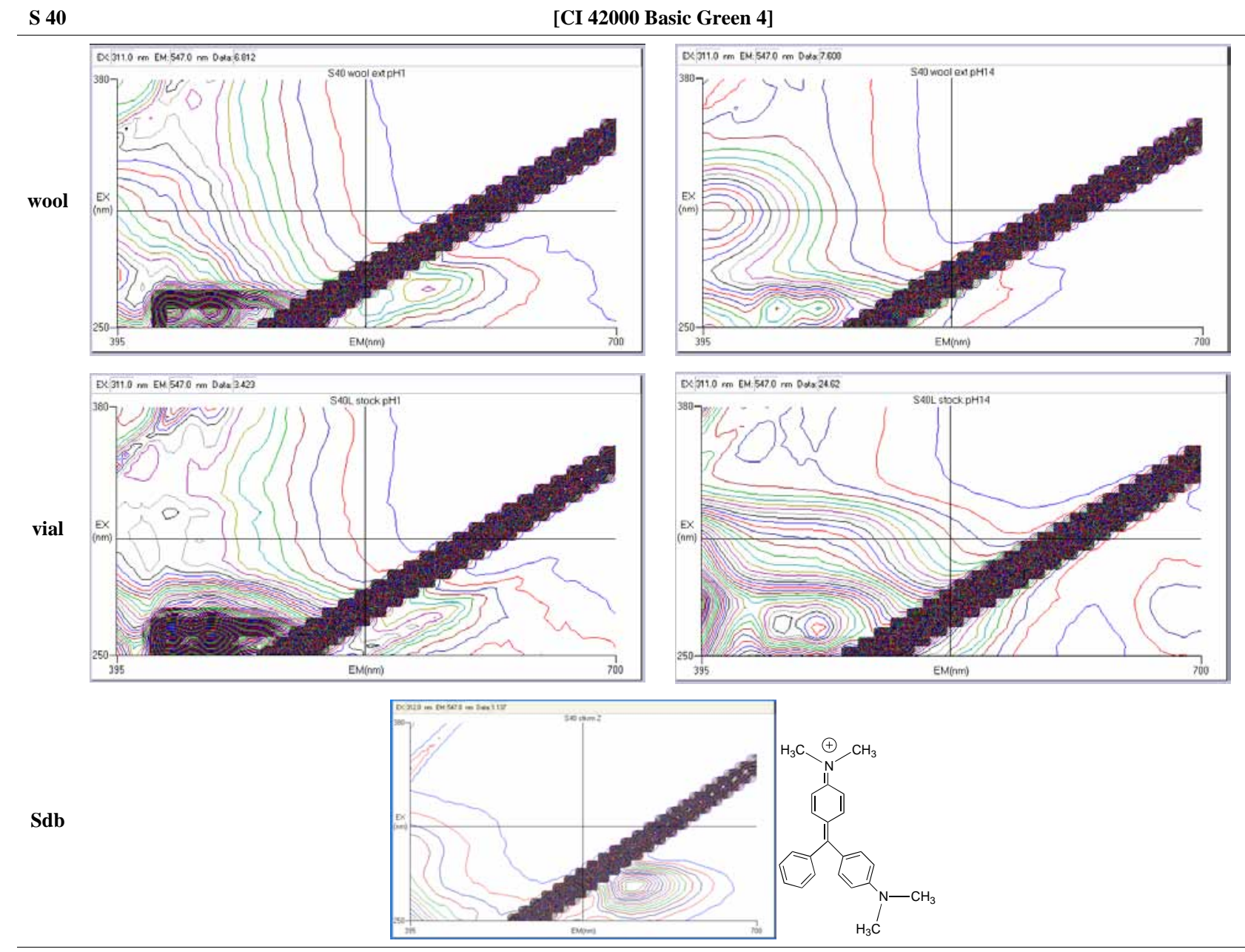

S 43

[CI 42075 Acid Green 6]
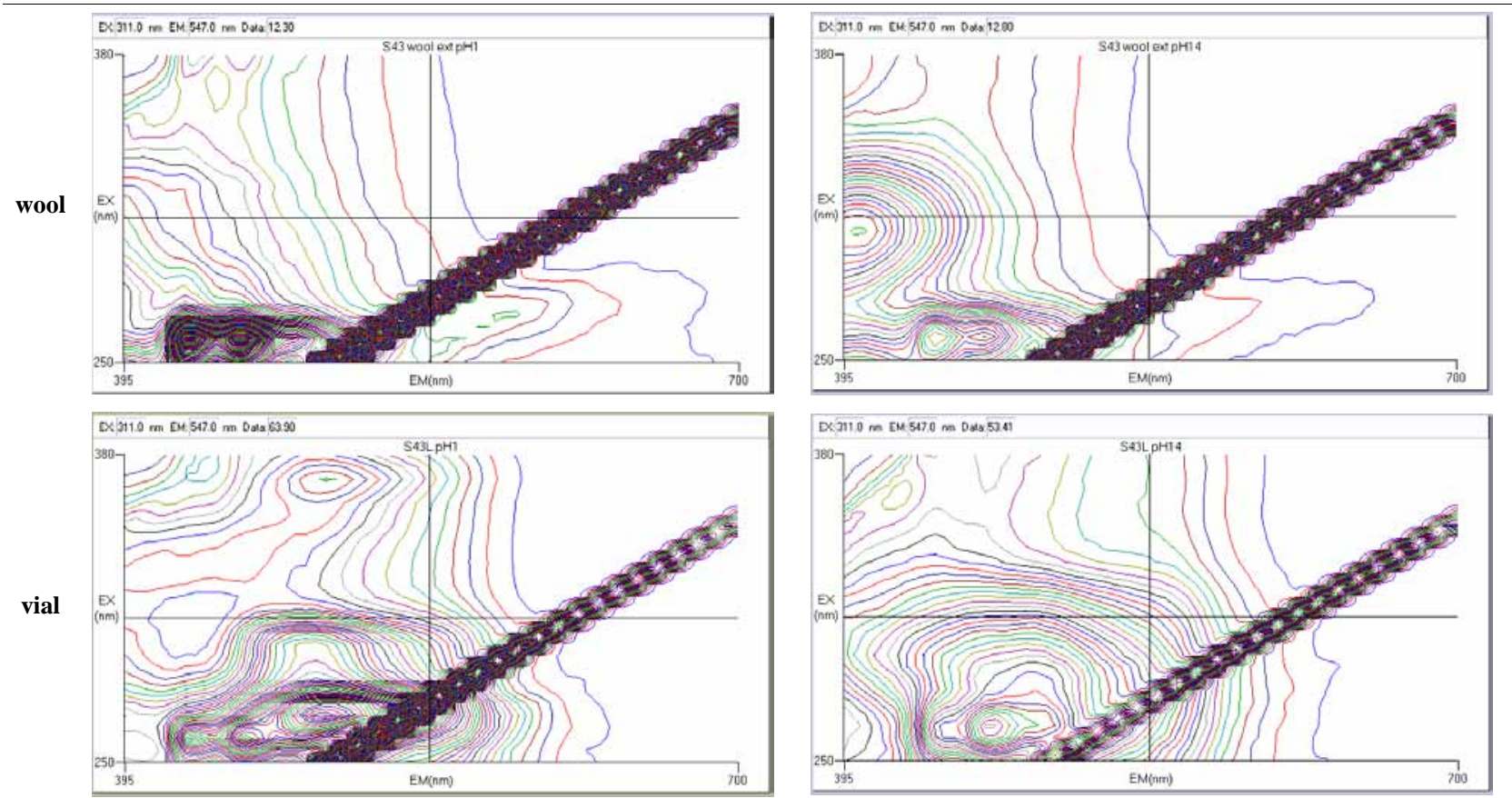


\section{Continued}
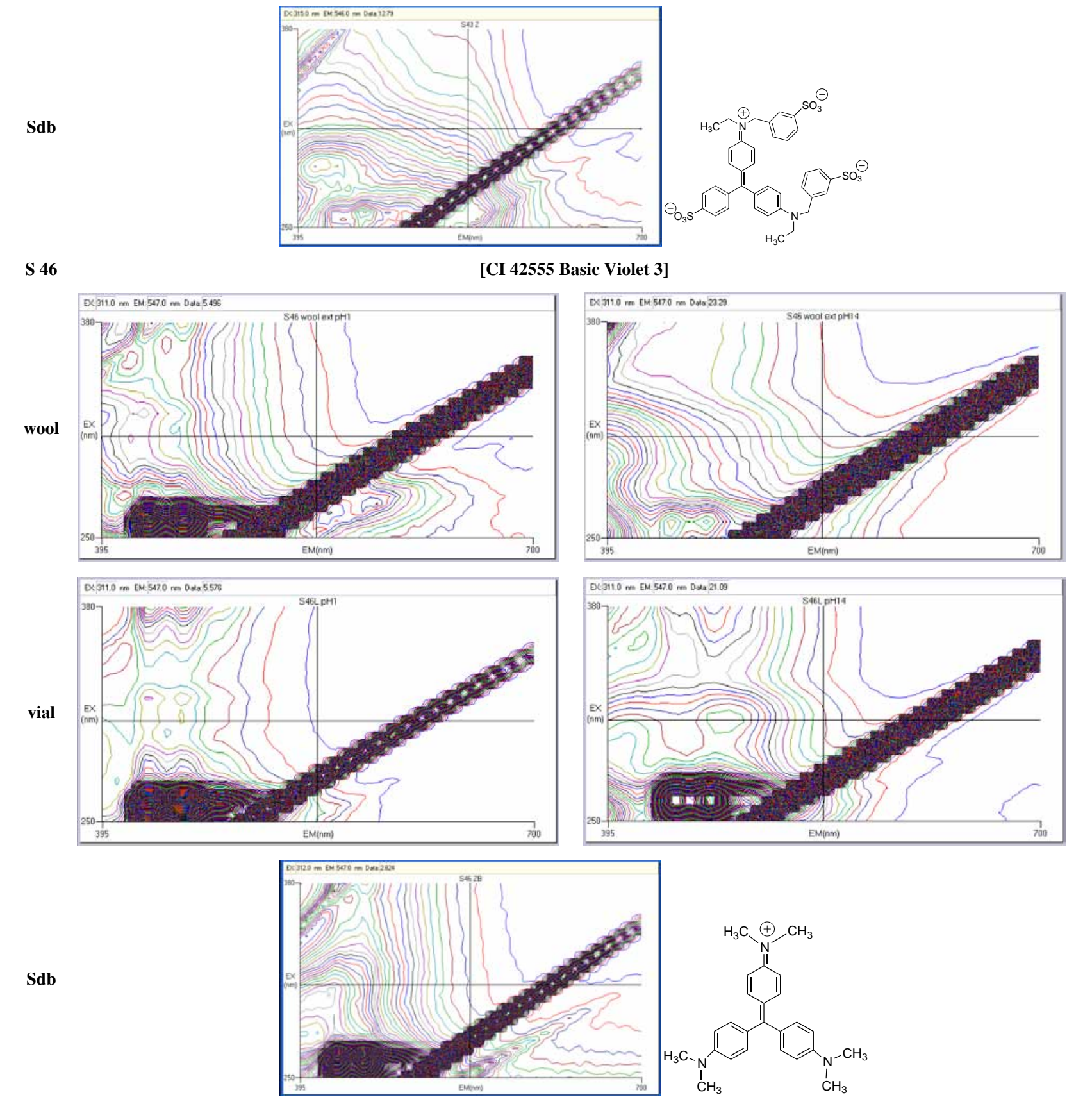

S 54

[CI 45430 Acid Red 51]
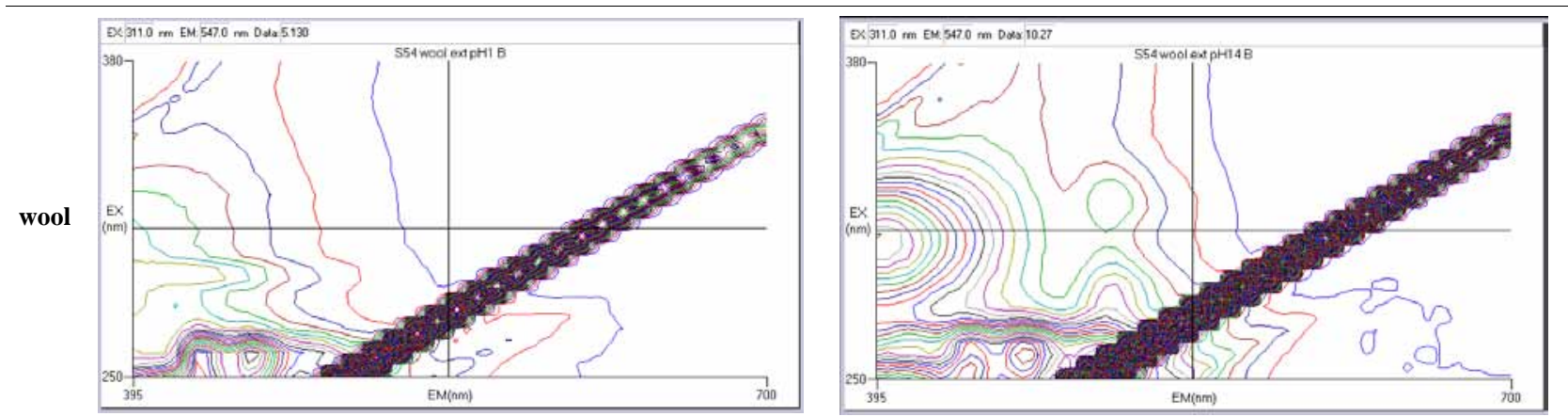


\section{Continued}
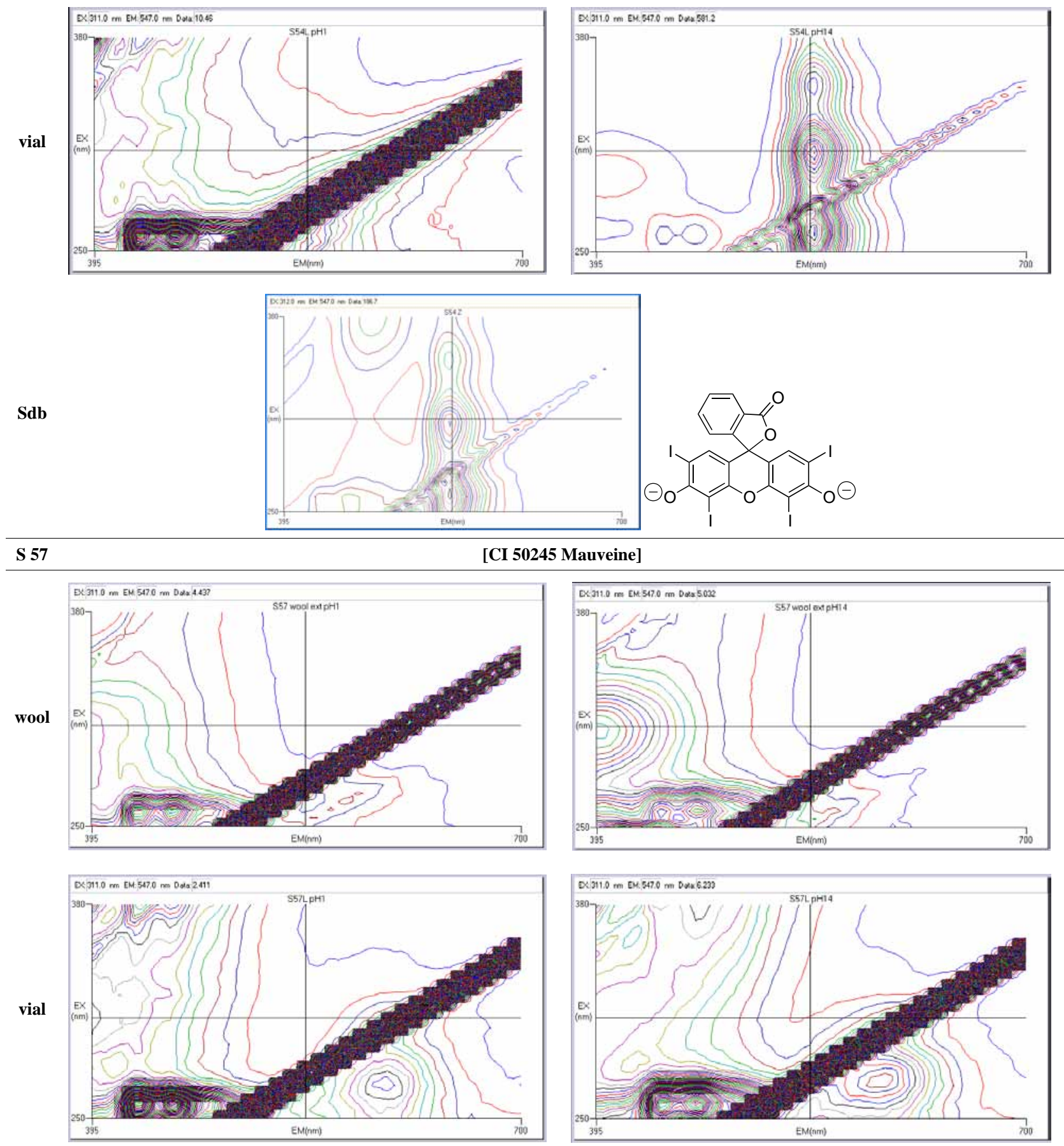

Sdb

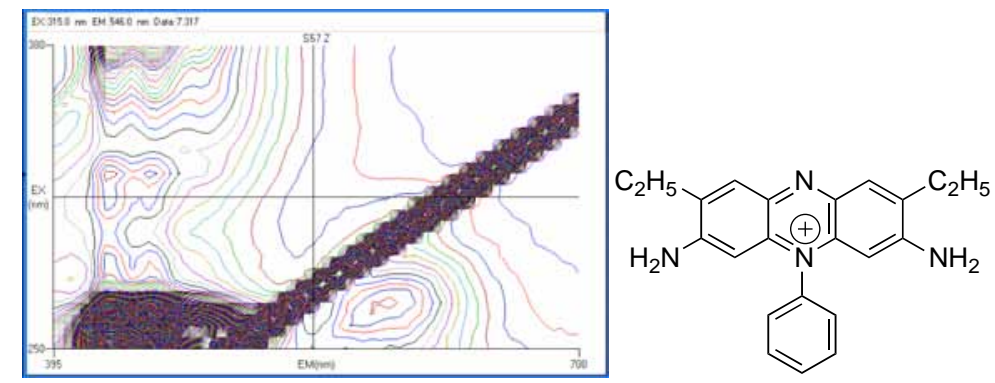




\section{Continued}

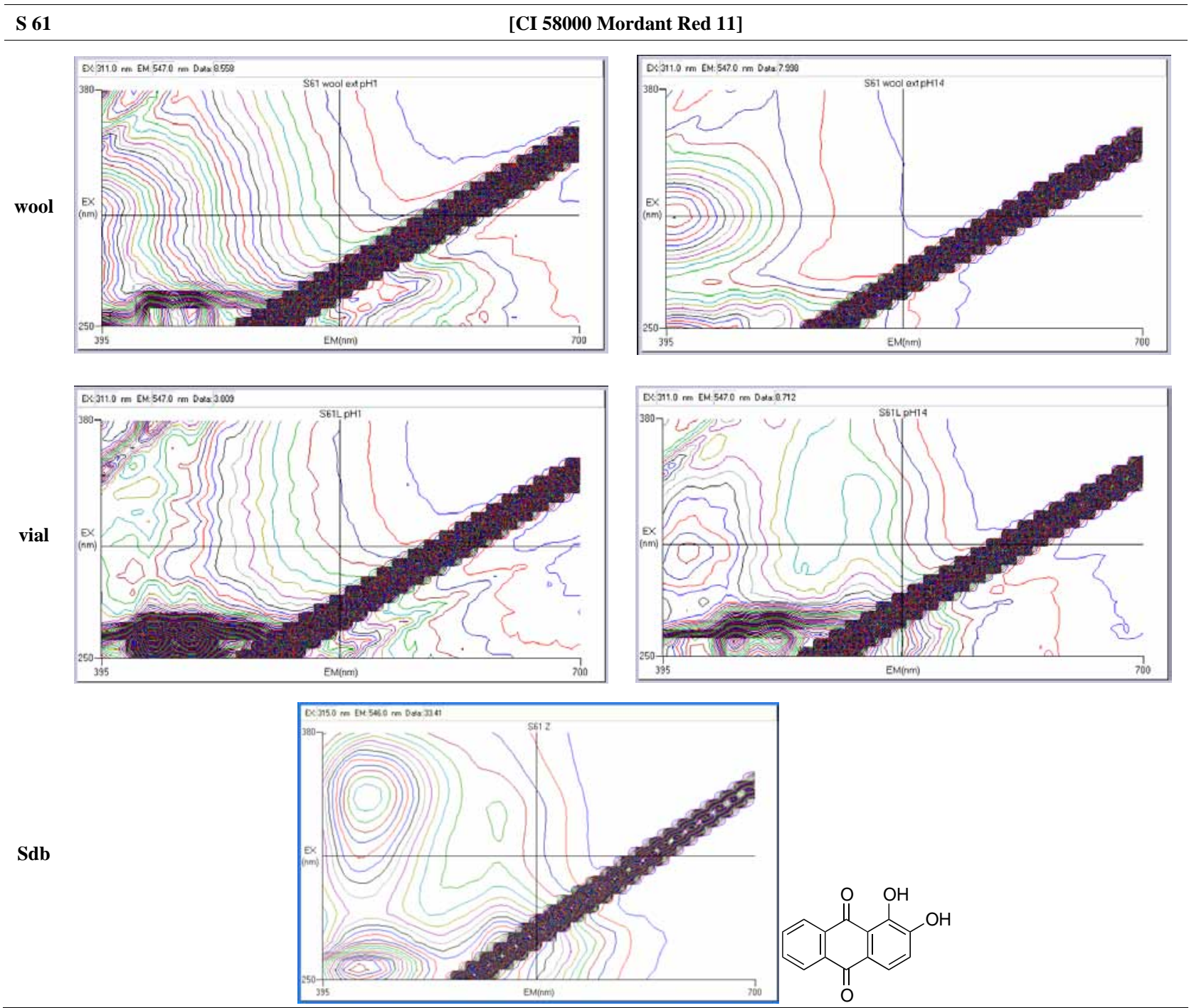

because of insufficient extraction.

\section{Conclusions}

Over $75 \%$ of the 65 synthetic organic dyes in the Schweppe collection can be successfully extracted from wool yarn under mild conditions and identified from their 3D fluorescence spectra at $\mathrm{pH} 1$ and/or pH 14. Only $2 \mathrm{mg}$ of wool is a sufficient sample size. There are three pairs of structurally similar dyes for which the spectra are too similar to allow differentiation (S 19/S 20, S 23/S 24 and S 25/S 26); in these cases, MALDI-TOF mass spectra would resolve the identity of the dyes [12].

\section{Acknowledgements}

We thank the Camille and Henry Dreyfus Foundation for a Senior Faculty Mentor grant supporting the participation of Kirch and Flynn. We also thank the National Sci- ence Foundation for grant CHE-0216268.

\section{REFERENCES}

[1] L. J. Soltzberg, S. Lor, N. Okey-Igwe and R. Newman, "3D Fluorescence Characterization of Synthetic Organic Dyes,” American Journal of Analytical Chemistry, Vol. 3, No. 9, 2012, pp. 622-631. http://dx.doi.org/10.4236/ajac.2012.39081

[2] "Schweppe Collection of Important Early Synthetic Dyes," Getty Conservation Institute, Los Angeles (The Collection Consists of Methanol Solutions of 65 dyes Assembled by the late Helmut Schweppe of BASF GmbH, as well as Fabric Swatches Dyed with These Substances).

[3] C. Souto, “Analysis of Early Synthetic Dyes with HPLCDAD-MS: An Important Database for Analysis of Colorants Used in Cultural Heritage,” Master's Thesis, 2010. http://run.unl.pt/bitstream/10362/5656/1/Souto_2010.pdf

[4] X. Zhang and R. A. Laursen, "Development of Mild Ex- 
traction Methods for the Analysis of Natural Dyes in Textiles of Historical Interest Using LC-Diode Array Detector-MS,” Analytical Chemistry, Vol. 77, No. 7, 2005, pp. 2022-2025. http://dx.doi.org/10.1021/ac048380k

[5] M. R. van Bommel, I. Vanden Berghe, A. M. Wallert, R. Boitelle and J. Wouters, "High-Performance Liquid Chromatography and Non-Destructive Three-Dimensional Fluorescence Analysis of Early Synthetic Dyes,” Journal of Chromatography A, Vol. 1157, No. 1-2, 2007, pp. 260272. http://dx.doi.org/10.1016/j.chroma.2007.05.017

[6] L. V. Belovolova, M. V. Glushkov, E. A. Vinogradov, V. A. Babintsev and V. I. Golovanov, "Ultraviolet Fluorescence of Water and Highly Diluted Aqueous Media," Physics of Wave Phenomena, Vol. 17, No. 1, 2009, pp. 21-31. http://dx.doi.org/10.3103/S1541308X0901004X

[7] K. Yasuhira and G. Takahashi, "Fluorometric Measurement of 3-Methylcholanthrene and Its Metabolites in Tissue. I. Measurement of 3-Methylcholanthrene in Organic Solvents,” Bulletin of the Chest Disease Research Institute, Vol. 8, 1974, pp. 1-7.
[8] E. L. Wehry, "Effects of Molecular Structure on Fluorescence and Phosphorescence” In: G. G. Guilbault, Practical Fluorescence, 2nd Edition, Marcel Dekker, New York, 1990, pp. 75-126.

[9] E. L. Wehry, "Effects of Molecular Environment on Fluorescence and Phosphorescence,” G. G. Guilbault, op. cit., pp. 127-184.

[10] "The Sigma-Aldrich Handbook of Stains, Dyes and Indicators,” Floyd. J. Green, Aldrich Chemical Company, Milwaukee, 1990.

[11] W. Knoche and N. Rees, "The Kinetics and Mechanism of the Decomposition of Murexide in Acid Solution," Journal of Chemical Education, Vol. 61, No. 8, 1984, pp. 724-726. http://dx.doi.org/10.1021/ed061p724

[12] L. J. Soltzberg, A. Hagar, S. Kridaratikorn, A. Mattson and R. Newman, "MALDI-TOF Mass Spectrometric Identification of Dyes and Pigments," Journal of the American Society for Mass Spectrometry, Vol. 18, No. 11, 2007, pp. 2001-2006. http://dx.doi.org/10.1016/j.jasms.2007.08.008 